\title{
Molecular Dynamics for Fermions
}

\author{
Hans Feldmeier \\ Gesellschaft für Schwerionenforschung mbH \\ D-64220 Darmstadt \\ Jürgen Schnack \\ Universität Osnabrück, Fachbereich Physik \\ Barbarastr. 7, D-49069 Osnabrück
}

\begin{abstract}
The time-dependent variational principle for many-body trial states is used to discuss the relation between the approaches of different molecular dynamics models to describe indistinguishable fermions. Early attempts to include effects of the Pauli principle by means of nonlocal potentials as well as more recent models which work with antisymmetrized many-body states are reviewed under these premises.

PACS: 02.70.Ns; 05.30.-d; 05.30.Ch; 05.30.Fk; 05.60.+w; 24.10.Cn Keywords: Many-body theory; Fermion system; Molecular dynamics; Wavepacket dynamics; Time-dependent variational principle; Statistical properties; Canonical ensemble; Ergodicity; Time averaging
\end{abstract}

To be published in July 2000 issue of Reviews of Modern Physics

(c) 2000 The American Physical Society 


\section{Contents}

I Introduction and Summary

$\begin{array}{lll}\text { II Time-dependent variational principle } & 8\end{array}$

A General remarks ........................ 8

1 Restricted set of variables . . . . . . . . . . . . . . . . . . 10

2 Deviation from the exact solution . . . . . . . . . . . . . . . . 13

3 Poisson brackets and canonical variables . . . . . . . . . . . . . . 15

4 Conservation laws . . . . . . . . . . . . . . . . 16

B From quantum to classical mechanics . . . . . . . . . . . . . . . . 18

1 Quantum mechanics ........................ 18

2 Classical mechanics . . . . . . . . . . . . . . . . . . . . . . . 19

3 Semi-classical, semi-quantal . . . . . . . . . . . . . . . 22

C Further remarks .......................... 24

1 Selfconsistency and nonlinearity . . . . . . . . . . . . . . 24

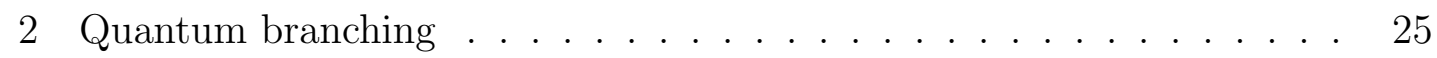

3 Approximation or new dynamical model? . . . . . . . . . . . . . . 28

$\begin{array}{lll}\text { III Antisymmetrization } & 31\end{array}$

A Effects of antisymmetrization in two-body space . . . . . . . . . . 33

1 Static considerations . . . . . . . . . . . . . . . . 33

2 Center of mass motion . . . . . . . . . . . . . . . . 38

3 Relative motion . . . . . . . . . . . . . . . . . . . 39

4 Relative motion with a time-independent width parameter . . . . . . . 41

B Effects of antisymmetrization in many-body space . . . . . . . . . 47

1 Shell structure due to antisymmetrization . . . . . . . . . . . . . . . . 47

2 Fermi-Dirac distribution due to antisymmetrization . . . . . . . . . . 48

3 Dynamical considerations . . . . . . . . . . . . . . . 5 50 
A Antisymmetrized wave packets in nuclear physics . . . . . . . . . . . . 52

1 Time-dependent $\alpha$-cluster model . . . . . . . . . . . . . . . . . . 53

2 Fermionic Molecular Dynamics - FMD . . . . . . . . . . . . . 54

3 Antisymmetrized Molecular Dynamics - AMD . . . . . . . . . . 55

B Product states of wave packets - QMD . . . . . . . . . . 58

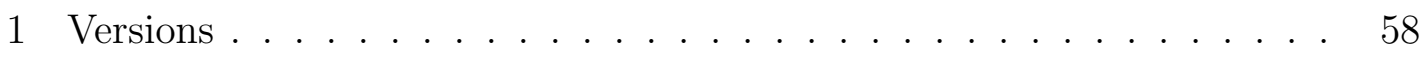

2 Decoupling of center of mass and relative motion . . . . . . . . . . 60

3 Approximate canonical variables - Pauli potential . . . . . . . . . . . . 61

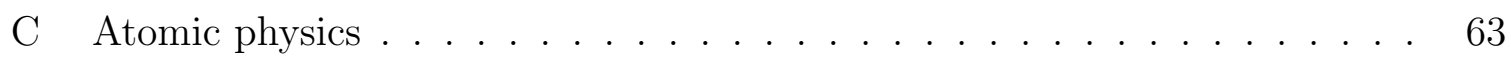

1 Product states of wave packets in atomic physics . . . . . . . . . . . 64

2 Quantum branching ....................... 65

$\begin{array}{lll}\text { V } & \text { Statistical properties } & 66\end{array}$

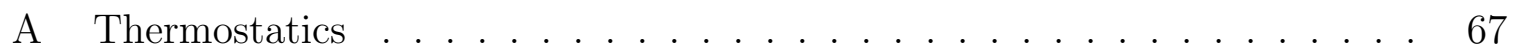

1 Completeness relation with coherent states . . . . . . . . . 68

2 Example for many fermions . . . . . . . . . . . . . . 71

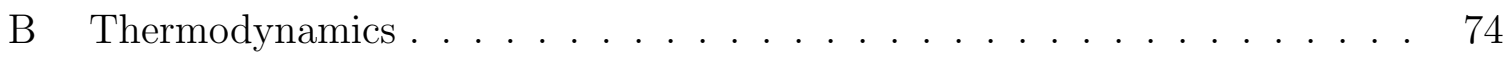

1 Ergodic ensemble of fermions in a harmonic oscillator . . . . . . . . . 75

2 Describing the system with fixed-width trial states . . . . . . . . . 77

3 Canonical and ergodic ensemble for distinguishable particles . . . . . . 79

C Thermal properties of interacting systems by time averaging . . . . . . . 81

1 FMD - the nuclear caloric curve . . . . . . . . . . . . . . . . . 82

2 Phase transitions of hydrogen plasma . . . . . . . . . . . . . 84

$\begin{array}{lr}\text { REFERENCES } & 87\end{array}$ 


\section{INTRODUCTION AND SUMMARY}

When correlations and fluctuations become important in the dynamical evolution of a many-body system and mean field approximations are not sufficient, molecular dynamics methods are invoked frequently. Molecular dynamics means that the constituents (molecules) of the many-body system are represented by few classical degrees of freedom (center of mass position and momentum, angles of rotation etc.) and interact through potentials. The equations of motion (Newton's or Hamilton's type) are solved numerically. The interactions which are used range from purely phenomenological to sophisticated ab initio quantal potentials. The major advantage of molecular dynamics simulations is that they do not rely on quasi-particle approximations but include both, mean-field effects and many-body correlations. Therefore they can provide insight into complex systems with correlations on different scales.

During the past decade one can observe an increasing interest in the dynamics of manyfermion systems in which correlations are important. In nuclear as well as in atomic physics collisions of composite fermion systems like nuclei or atomic clusters are demanding manybody models which can account for a large variety of phenomena. Depending on the energy one observes fusion, dissipative reactions, fragmentation and even multi-fragmentation, vaporisation or evaporation and ionization. Also phase transitions in small systems are of current interest.

Classical molecular dynamics is applicable if the de Broglie wave-length of the molecules is small compared to the length scale of typical variations of the interaction, otherwise the quantal uncertainty relation becomes important. If the molecules are identical fermions or bosons the de Broglie wave-length should also be small compared to the mean inter-particle distance in phase space, otherwise the Pauli or Bose principle is violated and the model will have wrong statistical properties.

For nucleons in a nucleus, for example, both conditions neccessary for classical mechanics are not fulfilled. The same holds for electrons in bound states or at high densities and low 
temperatures. Nevertheless one would like to utilize the merits of a molecular dynamics model for indistingushable particles in the quantum regime.

This article reviews attempts that are made to combine Fermi-Dirac statistics with a semi-quantal trajectory picture from the viewpoint of the quantal time-dependent variational principle. The closest quantum analogue to a point in single-particle phase space representing a classical particle is a wave packet well localized in phase space. The analogue to a point in many-body phase space representing several classical particles is a many-body state which is a product of localized single-particle packets. If the particles are identical fermions this product state has to be antisymmetrized, in the case of bosons it has to be symmetrized.

For models which are formulated in terms of trial states and a Hamilton operator, both, static and dynamical properties can be obtained from appropriate quantum variational principles. Ground states can be determined with the help of the Ritz variational principle and the equations of motion can be accessed through the time-dependent variational principle which allows to derive approximations to the time-dependent Schrödinger equation on different levels of accuracy.

In chapter II we first discuss the time-dependent variational principle in general and then show how it works for various trial states and how classical mechanics can be obtained from quantum mechanics by an appropriate choice of the dynamical variables.

Using wave packets automatically guarantees that the Heisenberg uncertainty principle is not violated by the model. That is actually a great problem in classical simulations of Coulomb systems, where for instance the hydrogen atom for a point like proton and electron is infinitely bound.

Using antisymmetrized many-body trial states automatically guarantees that the Pauli exclusion principle is respected by the model. Phenomena like shell structure or Fermi-Dirac statistics emerge in a natural way and are discussed in chapter III and V.

Although antisymmetrized product states of single-particle gaussian wave packets possess already major relevant degrees of freedom, some processes like disintegration of wave packets, 
which can occur in coordinate space (evaporation, capture or tunneling) and in momentum space (large momentum transfer due to collisions), are poorly described by the equations of motion. One therefore often represents the system with a mixture of trial states between which random transitions may occur. This branching procedure, which is employed in atomic as well as in nuclear physics, will be explained in Sec. II C 2.

In chapter IV models used in nuclear and atomic physics are reviewed from the general point of view of chapter II and III. When energetic collisions between heavy atomic nuclei became available classical molecular dynamics models were developed to describe the various phenomena observed. To simulate the effect of antisymmetrization on the classical trajectories many authors add to the hamiltonian a two-body "Pauli potential" which is supposed to keep fermions apart from each other in phase space.

Quantum Molecular Dynamics (QMD) attributes to each fermion a gaussian wave packet with fixed width instead of a point, but still uses a simple product state for the many-body wave function and therefore obtains classical equations of motion with two-body forces acting on the centroids of the wave packets. These Newtonian forces are supplemented by random forces which simulate hard collisions. In these collision terms Pauli blocking is included. The statistical properties are however mainly those of distinguishable particles.

Antisymmetrized Molecular Dynamics (AMD) also uses gaussian wave packets with fixed width, but antisymmetrizes the many-body state. Like in QMD a collision term is added to account in a phenomenological way for branching into other Slater determinants. In Fermionic Molecular Dynamics (FMD) in addition the width degree of freedom is considered. This non-classical degree of freedom is important for phenomena like evaporation and it also plays an important rôle in statistical properties of the model.

Section IV C explains the different use of the term Quantum Molecular Dynamics in atomic and nuclear physics. It then refers to applications of trajectory calculations for individual electrons and ions where the density of the electrons is too large to neglect their fermion character. Quantum branching in the atomic context is also briefly discussed.

Although designed for non-equilibrium simulations like collisions, molecular dynamics 
models are also used to simulate systems in thermal equilibrium. In chapter $\mathrm{V}$ their statistical properties are investigated by means of time averaging. As applications the nuclear liquid-gas phase transition or the hydrogen plasma under extreme conditions are discussed.

In conclusion, the review shows that it is possible to extend the classical trajectory picture to identical fermions by means of localized wave packets. When the phase space density increases the classical notion of positions $\vec{r}_{k}$ and momenta $\vec{p}_{k}$ as mean values of narrow wave packets has to be reinterpreted as parameters which identify an antisymmetrized manybody state $|Q\rangle=\left|\vec{r}_{1}, \vec{p}_{1}, \vec{r}_{2}, \vec{p}_{2}, \cdots\right\rangle$. When the individual packets overlap in phase space $\vec{r}_{k}$ and $\vec{p}_{k}$ can no longer be identified with the classical variables. Calculating all observables with the many-body state $|Q\rangle$ as quantum expectation values, e.g. $\mathcal{H}\left(\vec{r}_{1}, \vec{p}_{1}, \vec{r}_{2}, \vec{p}_{2}, \cdots\right)=$ $\langle Q|\underset{\sim}{H}| Q\rangle /\langle Q \mid Q\rangle$, and not misinterpreting them as classical expressions, includes the Pauli exclusion principle and Heisenberg's uncertainty principle in a natural and correct way. In the equations of motion the exclusion principle causes a complicated metric in the $N$-particle parameter space in the sense that canonical pairs of variables can only be defined locally.

The antisymmetrization of localized wave packets, which brings together the Pauli principle, Heisenberg's uncertainty relation and the classical trajectory picture, leads to many, some times unexpected, quantal features. Nevertheless one has to be aware that one is still dealing with a very simplified trial state and other degrees of freedom may be important. Especially when the interaction is not smooth across a wave packet it may want to change its shape to one which is not in the allowed set, for example split with certain amplitudes into different parts which after some time evolve independently. Or more generally, branching into other trial states away from the one which follows the approximate time evolution of a pure state leads to a mixture of antisymmetrized wave packets. The consistent treatment of this aspect of quantum branching needs further attention in the literature.

Common to all models discussed is the anticorrelation between its degree of consistent derivation and its computational effort. 


\section{TIME-DEPENDENT VARIATIONAL PRINCIPLE}

\section{A. General remarks}

The time evolution of a state in quantum mechanics is given by the time-dependent Schrödinger equation ${ }^{1}$

$$
i \frac{d}{d t}|\Psi(t)\rangle=\underset{\sim}{H}|\Psi(t)\rangle
$$

where $\underset{\sim}{H}$ is the hamiltonian and $|\Psi(t)\rangle$ the many-body state which describes the physical system. This equation of motion can be obtained from the variation of the action (Kerman and Koonin, 1976; Kramer and Saraceno, 1981; Drożdż et al., 1986; Broeckhove et al., 1988)

$$
\mathcal{A}^{\prime}=\int_{t_{1}}^{t_{2}} d t \mathcal{L}^{\prime}\left(\Psi(t)^{*}, \Psi(t), \dot{\Psi}(t)\right)
$$

keeping the variations fixed at the end points: $\delta \Psi\left(t_{1}\right)=\delta \Psi\left(t_{2}\right)=\delta \Psi^{*}\left(t_{1}\right)=\delta \Psi^{*}\left(t_{2}\right)=0$. The Lagrange function

$$
\mathcal{L}^{\prime}\left(\Psi(t)^{*}, \Psi(t), \dot{\Psi}(t)\right)=\left\langle\Psi(t)\left|i \frac{d}{d t}\right| \Psi(t)\right\rangle-\langle\Psi(t)|\underset{\sim}{H}| \Psi(t)\rangle
$$

is a function of the dynamical variables, denoted by the set $\Psi(t)$. Usually they are chosen to be complex so that $\Psi(t)$ and $\Psi^{*}(t)$ may be regarded as independent variables. If the mapping of $\Psi(t)$ on the many-body state $|\Psi(t)\rangle$ is analytic, $|\Psi(t)\rangle$ depends only on $\Psi(t)$ and the hermitian adjoint state $\langle\Psi(t)|$ only on the complex conjugate set $\Psi^{*}(t)$. The Lagrange function depends on the first time derivatives $\dot{\Psi}(t)$ through $\frac{d}{d t}|\Psi(t)\rangle$.

In general, the set $\Psi(t)$ contains infinitely many dynamical degrees of freedom, for example the complex coefficients of an orthonormal basis or the values of a $N$-body wave function on a $3 N$-dimensional grid in coordinate space.

\footnotetext{
${ }^{1}$ Throughout the article all operators are underlined with a tilde, e.g. $\underset{\sim}{H}$, and expectation values are denoted by caligraphic letters, e.g. $\mathcal{H}$. If not needed explicitly $\hbar$ is taken to be one.
} 
The Lagrange function (3) is appropriate if the set of variables $\Psi(t), \Psi^{*}(t)$ contains a complex overall factor to $|\Psi(t)\rangle$ which takes care of norm and phase. If $|\Psi(t)\rangle$ cannot be normalized by means of the set $\Psi(t)$ the Lagrange function defined in Eq. (14) should be used. We shall employ both forms.

For linearly independent variables $\Psi(t)$ and $\Psi^{*}(t)$ the variation of $\Psi^{*}(t)$ yields for the extremal action

$$
0=\delta \mathcal{A}^{\prime}=\int_{t_{1}}^{t_{2}} d t \quad\left\langle\delta \Psi(t)\left|i \frac{d}{d t}-\underset{\sim}{H}\right| \Psi(t)\right\rangle
$$

If $|\Psi(t)\rangle$ represents the most general state in Hilbert space, the variation $\langle\delta \Psi(t)|$ is unrestricted and Eq. (4) can only be fulfilled if

$$
\left(i \frac{d}{d t}-\underset{\sim}{H}\right)|\Psi(t)\rangle=0
$$

which is just the Schrödinger equation (1).

The variation of $\Psi(t)$ yields

$$
0=\delta \mathcal{A}^{\prime}=\int_{t_{1}}^{t_{2}} d t \quad\left\langle\Psi(t)\left|i \frac{d}{d t}-\underset{\sim}{H}\right| \delta \Psi(t)\right\rangle
$$

which after partial integration over $t$ with fixed endpoints $\left|\delta \Psi\left(t_{1}\right)\right\rangle=\left|\delta \Psi\left(t_{2}\right)\right\rangle=0$ results in the hermitian adjoint of the Schrödiger equation

$$
\langle\Psi(t)|\left(-i \frac{\overleftarrow{d}}{d t}-\overleftarrow{\sim} \underset{\sim}{H}\right)=0
$$

As $\Psi(t)$ is linearly independent of $\Psi^{*}(t)$, both have to be varied. Therefore the time integral which was missing in early attempts, used by (Frenkel, 1934) and others, is necessary.

The reason for the reformulation of a differential equation as a variational principle is of course the anticipation that a suitably chosen restriction in the dynamical variables will lead to a useful approximation of the full problem. For more general considerations on the construction of variational principles see (Gerjuoy, Rau, and Spruch, 1983; Balian and Veneroni, 1988) and references therein. 


\section{Restricted set of variables}

Let a restricted choice of variables be denoted by the complex set $Q^{\prime}(t)=$ $\left\{q_{0}(t), q_{1}(t), q_{2}(t), \cdots\right\}$ which specifies the many-body state $\left|Q^{\prime}(t)\right\rangle$. It is presumed that $q_{0}(t)$ is always a complex overall factor in the sense

$$
\left|Q^{\prime}(t)\right\rangle=q_{0}(t)|Q(t)\rangle=q_{0}(t)\left|q_{1}(t), q_{2}(t), \cdots\right\rangle .
$$

Furthermore, the time-dependence of $\left|Q^{\prime}(t)\right\rangle$ is supposed to be only implicit through the variables $Q^{\prime}(t)$.

It should be noted that the manifold $\left|Q^{\prime}(t)\right\rangle$ is in general only a subset of the Hilbert space and needs not to form a subspace.

Variation of the action (2) with the Lagrange function (3) with respect to $Q^{\prime *}(t)$ leads to

$$
0=\delta \mathcal{A}^{\prime}=\int_{t_{1}}^{t_{2}} d t \sum_{\nu} \delta q_{\nu}^{*}(t)\left(\frac{\partial}{\partial q_{\nu}^{*}}\left\langle Q^{\prime}(t)\right|\right)\left(i \frac{d}{d t}-\underset{\sim}{H}\right)\left|Q^{\prime}(t)\right\rangle .
$$

As $\delta q_{\nu}^{*}(t)$ are arbitrary functions the action is extremal if the following equations of motion are fulfilled

$$
\left(\frac{\partial}{\partial q_{\nu}^{*}}\left\langle Q^{\prime}(t)\right|\right)\left(i \frac{d}{d t}-\underset{\sim}{H}\right)\left|Q^{\prime}(t)\right\rangle=0
$$

or

$$
i \sum_{\mu} \mathcal{C}_{\nu \mu}^{\prime} \dot{q}_{\mu}=\frac{\partial}{\partial q_{\nu}^{*}}\left\langle Q^{\prime}(t)|\underset{\sim}{H}| Q^{\prime}(t)\right\rangle
$$

where

$$
\mathcal{C}_{\nu \mu}^{\prime}=\frac{\partial^{2}}{\partial q_{\nu}^{*} \partial q_{\mu}}\left\langle Q^{\prime}(t) \mid Q^{\prime}(t)\right\rangle
$$

Different from the unrestricted variation one obtains equations of motion for the complex parameters which in turn define the time evolution of the trial state $\left|Q^{\prime}(t)\right\rangle$ in Hilbert space.

After partial integration over time variation with respect to $Q^{\prime}(t)$, with fixed end points, $\delta q_{\nu}\left(t_{1}\right)=\delta q_{\nu}\left(t_{2}\right)=0$, results in equations of motion which are the complex conjugate of Eqs. (10) - (12). 
In the following, for sake of simplicity, the explicit indication of the time dependence is sometimes omitted.

The time evolution of $q_{0}$ can be expressed in terms of the other variables. For that the Lagrangian $\mathcal{L}^{\prime}$ may be written as

$$
\begin{aligned}
\mathcal{L}^{\prime} & =\left\langle Q^{\prime}\left|i \frac{d}{d t}-\underset{\sim}{H}\right| Q^{\prime}\right\rangle \\
& =i q_{0}^{*} \dot{q}_{0}\langle Q \mid Q\rangle+q_{0}^{*} q_{0}\langle Q \mid Q\rangle \mathcal{L}\left(Q, Q^{*}, \dot{Q}, \dot{Q}^{*}\right)-\frac{i}{2} \frac{d}{d t}\left(q_{0}^{*} q_{0}\langle Q \mid Q\rangle\right),
\end{aligned}
$$

where the set $Q$ does not contain $q_{0}$ anymore (see Eq. (8)) and a new Lagrangian $\mathcal{L}\left(Q^{*}, Q, \dot{Q}^{*}, \dot{Q}\right)$ is defined by

$$
\begin{aligned}
\mathcal{L}\left(Q^{*}, Q, \dot{Q}^{*}, \dot{Q}\right) & =\frac{i}{2}\left(\frac{\langle Q \mid \dot{Q}\rangle-\langle\dot{Q} \mid Q\rangle}{\langle Q \mid Q\rangle}\right)-\frac{\langle Q|\underset{\sim}{H}| Q\rangle}{\langle Q \mid Q\rangle} \\
& \equiv \mathcal{L}_{0}\left(Q^{*}, Q, \dot{Q}^{*}, \dot{Q}\right)-\mathcal{H}\left(Q^{*}, Q\right)
\end{aligned}
$$

with

$$
|\dot{Q}\rangle \equiv \frac{d}{d t}|Q\rangle=\sum_{\nu} \dot{q}_{\nu} \frac{\partial}{\partial q_{\nu}}|Q\rangle \quad \text { and } \quad\langle\dot{Q}| \equiv \frac{d}{d t}\langle Q|=\sum_{\nu} \dot{q}_{\nu}^{*} \frac{\partial}{\partial q_{\nu}^{*}}\langle Q|
$$

The new Lagrange function $\mathcal{L}$ contains the norm explicitly and is made real by subtracting the total time derivative. $\mathcal{H}\left(Q^{*}, Q\right)$ is the expectation value of the hamiltonian $\underset{\sim}{H}$ and will be called Hamilton function.

It is easy to verify that the solution of the equation of motion (10) for $q_{0}$ is

$$
q_{0}(t)=\frac{1}{\langle Q(t) \mid Q(t)\rangle^{\frac{1}{2}}} \exp \left\{i \int^{t} d t^{\prime} \mathcal{L}\left(t^{\prime}\right)\right\}
$$

and analogue for the complex conjugate $q_{0}^{*}(t)$. Thus, the variational freedom of an overall factor $q_{0}(t)$ is used by the time-dependent variational principle to provide a state $\left|Q^{\prime}(t)\right\rangle$ with a time-independent norm and an the additional phase $\int^{t} d t^{\prime} \mathcal{L}\left(t^{\prime}\right)(\mathcal{L}$ is real by construction). Furthermore, insertion of the solution (16) into $\mathcal{L}^{\prime}$ shows that along the trajectory

$$
\mathcal{L}^{\prime}\left(Q^{\prime *}(t), Q^{\prime}(t), \dot{Q}^{\prime}(t)\right)=0
$$

irrespective of the choice of the remaining degrees of freedom in $|Q\rangle$. 
The equations of motion (10) and their complex conjugate ones can of course also be expressed as Euler Lagrange equations

$$
\frac{d}{d t} \frac{\partial \mathcal{L}^{\prime}}{\partial \dot{q}_{\nu}^{*}}-\frac{\partial \mathcal{L}^{\prime}}{\partial q_{\nu}^{*}}=0 \quad \text { and } \quad \frac{d}{d t} \frac{\partial \mathcal{L}^{\prime}}{\partial \dot{q}_{\nu}}-\frac{\partial \mathcal{L}^{\prime}}{\partial q_{\nu}}=0
$$

For the remaining variables $\left\{q_{\nu}^{*}, q_{\nu} ; \nu \neq 0\right\}$ they can be written in terms of the new Lagrange function $\mathcal{L}$ as

$$
\begin{aligned}
\frac{d}{d t} \frac{\partial \mathcal{L}^{\prime}}{\partial \dot{q}_{\nu}^{*}}-\frac{\partial \mathcal{L}^{\prime}}{\partial q_{\nu}^{*}}= & \left(\frac{d}{d t} \frac{\partial \mathcal{L}}{\partial \dot{q}_{\nu}^{*}}-\frac{\partial \mathcal{L}}{\partial q_{\nu}^{*}}\right) q_{0}^{*} q_{0}\langle Q \mid Q\rangle+ \\
& \frac{d}{d t}\left(q_{0}^{*} q_{0}\langle Q \mid Q\rangle\right) \frac{\partial \mathcal{L}}{\partial \dot{q}_{\nu}^{*}}-\left(i q_{0}^{*} \dot{q} q_{0}+q_{0}^{*} q_{0} \mathcal{L}\right) \frac{\partial\langle Q \mid Q\rangle}{\partial q_{\nu}^{*}}=0
\end{aligned}
$$

and the analogue complex conjugate ones. The last two terms vanish when the general solution (16) for $q_{0}$ is inserted. Hence for $\nu \neq 0$ the Euler Lagrange equations with $\mathcal{L}$ as given in (14) are equivalent to the ones with $\mathcal{L}^{\prime}$.

Therefore, the action

$$
\mathcal{A}=\int_{t_{1}}^{t_{2}} d t \mathcal{L}\left(Q^{*}, Q, \dot{Q}^{*}, \dot{Q}\right)
$$

which one also finds often as a starting point (Kerman and Koonin, 1976; Kramer and Saraceno, 1981; Drożdż et al., 1986; Broeckhove et al., 1988), is the appropriate one if the trial state $|Q\rangle$ is not normalized and the phase is disregarded ${ }^{2}$. This form will turn out to be more convenient when dealing with antisymmetrized states in section III.

One should however keep in mind that the simple inclusion of $q_{0}$ provides automatically norm and phase.

The Euler Lagrange equations, which result from variation of the action (20)

$$
\frac{d}{d t} \frac{\partial \mathcal{L}}{\partial \dot{q}_{\nu}^{*}}=\frac{\partial \mathcal{L}}{\partial q_{\nu}^{*}} \quad \text { and } \quad \frac{d}{d t} \frac{\partial \mathcal{L}}{\partial \dot{q}_{\nu}}=\frac{\partial \mathcal{L}}{\partial q_{\nu}}
$$

\footnotetext{
${ }^{2} \mathcal{L}$ may also be written with normalized trial states $|Q\rangle / \sqrt{\langle Q \mid Q\rangle}$. The only difference is a total time derivative emerging from $\mathcal{L}_{0}$ so that the Lagrange functions are equivalent.
} 
can be written in terms of the Hamilton function $\mathcal{H}=\langle Q|\underset{\sim}{H}| Q\rangle /\langle Q \mid Q\rangle$ as generalized Hamilton's equations

$$
i \sum_{\nu} \mathcal{C}_{\mu \nu} \dot{q}_{\nu}=\frac{\partial \mathcal{H}}{\partial q_{\mu}^{*}} \quad \text { and } \quad-i \sum_{\nu} \mathcal{C}_{\mu \nu}^{*} \dot{q}_{\nu}^{*}=\frac{\partial \mathcal{H}}{\partial q_{\mu}}
$$

The nonnegative hermitian matrix $\mathcal{C}$ depends in general on $Q$ and is given by

$$
\mathcal{C}_{\mu \nu}=\frac{\frac{\partial}{\partial q_{\mu}^{*}} \frac{\partial}{\partial q_{\nu}}\langle Q \mid Q\rangle}{\langle Q \mid Q\rangle}-\frac{\frac{\partial}{\partial q_{\mu}^{*}}\langle Q \mid Q\rangle}{\langle Q \mid Q\rangle} \frac{\frac{\partial}{\partial q_{\nu}}\langle Q \mid Q\rangle}{\langle Q \mid Q\rangle}=\frac{\partial}{\partial q_{\mu}^{*}} \frac{\partial}{\partial q_{\nu}} \ln \langle Q \mid Q\rangle .
$$

It plays the rôle of a metric on the manifold of dynamical variables $Q$.

There is no need to assume that the set $Q$ contains only complex variables. But as will be seen later in the context of classical mechanics, the real and imaginary parts of $q_{\nu}$ play the rôle of canonical pairs of variables. On the other hand for quantum mechanics it is only natural to work with complex variables as the quantum state $|Q\rangle$ is necessarily complex. The assumption of an analytic mapping of $Q$ onto the trial state $|Q\rangle$ is also not compulsory but makes the equations usually more transparent.

\section{Deviation from the exact solution}

The static analogue to the time-dependent variational principle is the Rayleigh-Ritz variational principle for the energy. Here one can show that the overlap between the true ground state of the hamiltonian and the trial state $\left|Q^{\prime}\right\rangle$ is increasing when the energy $\left\langle Q^{\prime}|\underset{\sim}{H}| Q^{\prime}\right\rangle$ is decreasing. Since any additional degree of freedom lowers the energy, more degrees of freedom imply always an improved description. General statements like that cannot be made for the time-dependent variational principle, although one would believe that more degrees of freedom will result in a time evolution which is closer to that of the Schrödinger equation.

In order to understand better in which sense the time-dependent variational principle optimizes the evolution of the trial state, we consider the deviation of the approximate solution from the exact one, which develops during a short time $\delta t$ 


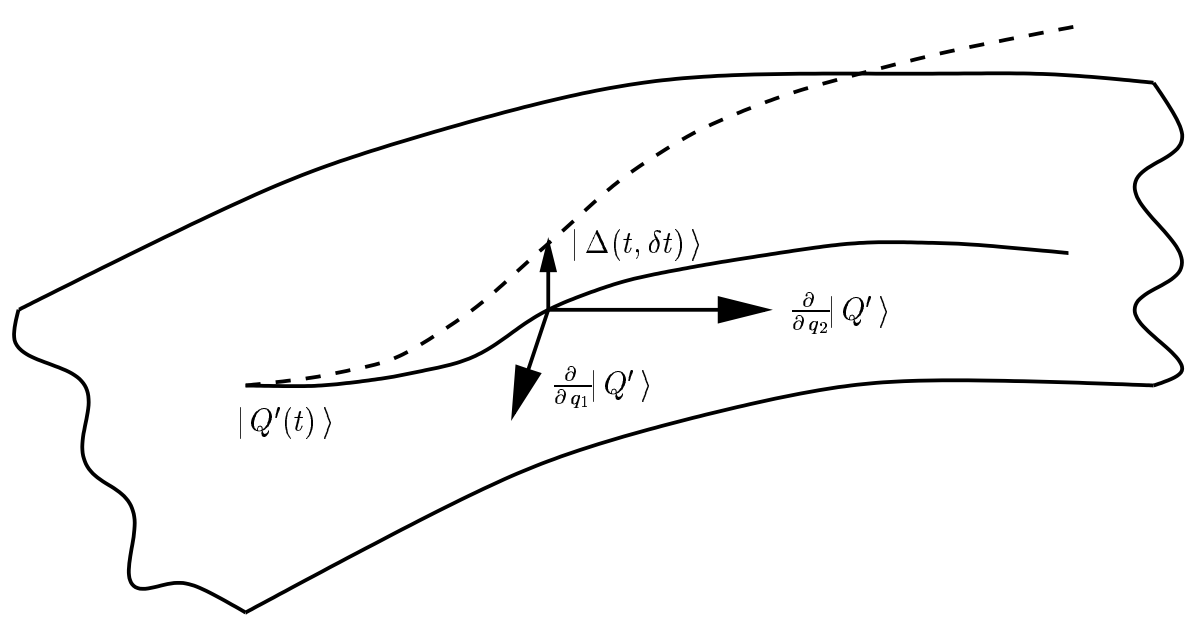

FIG. 1. Sketch of the manifold of trial states with the approximate time evolution (solid line) and the solution of the Schrödinger equation (dashed line). The error $|\Delta(t, \delta t)\rangle$ is orthogonal to all tangent states $\frac{\partial}{\partial q_{\nu}}\left|Q^{\prime}\right\rangle$.

$$
\begin{aligned}
|\Delta(t, \delta t)\rangle & =\left|\Psi_{\text {exact }}(t+\delta t)\right\rangle-\left|Q^{\prime}(t+\delta t)\right\rangle=\exp (-i \underset{\sim}{H} \delta t)\left|Q^{\prime}(t)\right\rangle-\left|Q^{\prime}(t+\delta t)\right\rangle \\
& =-i\left(\underset{\sim}{H}-i \sum_{\nu} \dot{q}_{\nu} \frac{\partial}{\partial q_{\nu}}\right)\left|Q^{\prime}(t)\right\rangle \delta t+\operatorname{order}\left(\delta t^{2}\right) .
\end{aligned}
$$

The equations of motion (10) which result from the time-dependent variational principle demand that

$$
\left(\frac{\partial}{\partial q_{\nu}^{*}}\left\langle Q^{\prime}(t)\right|\right)|\Delta(t, \delta t)\rangle=0
$$

which means that the deviation is orthogonal to all tangent states $\frac{\partial}{\partial q_{\nu}}\left|Q^{\prime}\right\rangle$, see Fig. 1. In other words the approximate equations of motions evolve $Q^{\prime}(t)$ to that point $Q^{\prime}(t+\delta t)$ in the manifold where any small change in all possible directions increases the distance $\langle\Delta(t, \delta t) \mid \Delta(t, \delta t)\rangle$ between true $\left|\Psi_{\text {exact }}(t+\delta t)\right\rangle$ and approximate solution $\left|Q^{\prime}(t+\delta t)\right\rangle$.

The minimum condition

$$
\begin{aligned}
0 & =\frac{\partial}{\partial q_{\nu}^{*}(t+\delta t)}\langle\Delta(t, \delta t) \mid \Delta(t, \delta t)\rangle \\
& =-\left(\frac{\partial}{\partial q_{\nu}^{*}(t+\delta t)}\left\langle Q^{\prime}(t+\delta t)\right|\right)|\Delta(t, \delta t)\rangle
\end{aligned}
$$




$$
=-\left(\frac{\partial}{\partial q_{\nu}^{*}(t)}\left\langle Q^{\prime}(t)\right|\right)|\Delta(t, \delta t)\rangle+\operatorname{order}\left(\delta t^{3}\right) .
$$

is fulfilled because of Eq. (25). For variations with respect to $q_{\nu}$ the complex conjugate of Eqs. (25) and (26) have to be used. Of course after many time steps $\delta t$ the deviation may become large.

\section{Poisson brackets and canonical variables}

With the help of $\mathcal{C}$ it is possible to introduce Poisson brackets. Using the equations of motion (22) the time derivative of the expectation value of a time-independent observable $\underset{\sim}{B}$ can be written as

$$
\begin{aligned}
\dot{\mathcal{B}} & =\frac{d}{d t} \frac{\langle Q|\underset{\sim}{B}| Q\rangle}{\langle Q \mid Q\rangle}=\sum_{\nu}\left(\dot{q}_{\nu}^{*} \frac{\partial \mathcal{B}}{\partial q_{\nu}^{*}}+\dot{q}_{\nu} \frac{\partial \mathcal{B}}{\partial q_{\nu}}\right) \\
& =i \sum_{\mu, \nu}\left(\frac{\partial \mathcal{H}}{\partial q_{\mu}} \mathcal{C}_{\mu \nu}^{-1} \frac{\partial \mathcal{B}}{\partial q_{\nu}^{*}}-\frac{\partial \mathcal{B}}{\partial q_{\mu}} \mathcal{C}_{\mu \nu}^{-1} \frac{\partial \mathcal{H}}{\partial q_{\nu}^{*}}\right) \\
& =:\{\mathcal{H}, \mathcal{B}\} .
\end{aligned}
$$

The real and imaginary parts of $q_{\nu}$ form pairs of canonical variables if $\mathcal{C}$ is the unit matrix. In the following section II B two examples are given for this case, the Schrödinger equation and Hamilton's equation of motion.

In the general case, in which $\mathcal{C}_{\mu \nu}$ is not diagonal and depends on $Q$, pairs of canonical variables exist locally according to Darboux's theorem (Arnol'd, 1989). One possible transformation is given by

$$
\mathrm{d} r_{\mu}=\sum_{\nu} \mathcal{C}_{\mu \nu}^{1 / 2}\left(Q^{*}, Q\right) \mathrm{d} q_{\nu} \quad \text { and } \quad \mathrm{d} r_{\mu}^{*}=\sum_{\nu} \mathrm{d} q_{\nu}^{*} \mathcal{C}_{\mu \nu}^{1 / 2}\left(Q^{*}, Q\right)
$$

Written with the new variables $r_{\mu}$ and $r_{\mu}^{*}$ the Poisson bracket (27) takes the form

$$
\{\mathcal{H}, \mathcal{B}\}=i \sum_{\mu}\left(\frac{\partial \widehat{\mathcal{H}}}{\partial r_{\mu}} \frac{\partial \widehat{\mathcal{B}}}{\partial r_{\mu}^{*}}-\frac{\partial \widehat{\mathcal{B}}}{\partial r_{\mu}} \frac{\partial \widehat{\mathcal{H}}}{\partial r_{\mu}^{*}}\right)
$$

so that $\left(r_{\mu}, r_{\mu}^{*}\right)$ form pairs of canonical variables. The problem is, however, that in nontrivial cases the transformation (28) cannot be written in a global way as $R(Q)=$ 
$\left\{r_{0}\left(q_{0}, q_{1}, \cdots\right), r_{1}\left(q_{0}, q_{1}, \cdots\right), \cdots\right\}$ and the Hamilton function $\mathcal{H}$ or the observable $\mathcal{B}$ cannot be expressed in the new variables

$$
\widehat{\mathcal{H}}\left(R^{*}, R\right)=\mathcal{H}\left(Q^{*}, Q\right) \quad, \quad \widehat{\mathcal{B}}\left(R^{*}, R\right)=\mathcal{B}\left(Q^{*}, Q\right)
$$

A set of canonical pairs which are real is given by

$$
\rho_{\mu}=\frac{1}{\sqrt{2}}\left(r_{\mu}^{*}+r_{\mu}\right) \quad \text { and } \quad \pi_{\mu}=\frac{i}{\sqrt{2}}\left(r_{\mu}^{*}-r_{\mu}\right)
$$

which yields the standard Poisson brackets

$$
\{\mathcal{H}, \mathcal{B}\}=\sum_{\mu}\left(\frac{\partial \widehat{\widehat{\mathcal{H}}}}{\partial \pi_{\mu}} \frac{\partial \widehat{\widehat{\mathcal{B}}}}{\partial \rho_{\mu}}-\frac{\partial \widehat{\widehat{\mathcal{B}}}}{\partial \pi_{\mu}} \frac{\partial \widehat{\widehat{\mathcal{H}}}}{\partial \rho_{\mu}}\right)
$$

where $\widehat{\widehat{\mathcal{H}}}$ and $\widehat{\widehat{\mathcal{B}}}$ are now functions of $\rho_{\mu}$ and $\pi_{\mu}$. Besides the trivial examples discussed in Sec. II B we give one nontrivial example in Sec. III A 3.

The mere fact that according to Darboux's theorem canonical pairs $\left(\rho_{\mu}, \pi_{\mu}\right)$ exist, admits to guess or make an ansatz for the Hamilton function $\widehat{\widehat{\mathcal{H}}}\left(\rho_{1}, \rho_{2}, \ldots, \pi_{1}, \pi_{2}, \ldots\right)$ and use Hamilton's equations of motion. But with such a guess the connection to the trial state $|Q\rangle$ is lost and the physical meaning of $\rho_{\mu}$ and $\pi_{\mu}$ is obscured. This will become obvious when we discuss trial states for indistinguishable particles in Sec. III.

\section{Conservation laws}

An expectation value is conserved if (Broeckhove et al., 1989)

$$
\dot{\mathcal{B}}=\{\mathcal{H}, \mathcal{B}\}=i \sum_{\mu, \nu}\left(\frac{\partial \mathcal{H}}{\partial q_{\mu}} \mathcal{C}_{\mu \nu}^{-1} \frac{\partial \mathcal{B}}{\partial q_{\nu}^{*}}-\frac{\partial \mathcal{B}}{\partial q_{\mu}} \mathcal{C}_{\mu \nu}^{-1} \frac{\partial \mathcal{H}}{\partial q_{\nu}^{*}}\right)=0
$$

Hence the energy $\mathcal{H}$ itself is always conserved by the equations of motion, provided they are derived from the variational principle. This is completely independent on the choice of the trial state.

In the following it is shown how to identify other constants of motion and how the trial state has to be chosen in order to ensure desired conservation laws. For that we consider a unitary transformation with the hermitian generator $\underset{\sim}{G}$ 


$$
\underset{\sim}{U}=\exp (i \varepsilon \underset{\sim}{G}) \quad, \quad \varepsilon \quad \text { real }
$$

If $\underset{\sim}{U}$ maps the set of trial states onto itself

$$
\underset{\sim}{U}\left|Q^{\prime}\right\rangle \in\left\{\left|Q^{\prime}\right\rangle\right\}
$$

then the special infinitesimal variation $\left|Q^{\prime}(t)+\delta Q^{\prime}(t)\right\rangle=\exp \{i \delta \varepsilon(t) \underset{\sim}{G}\}\left|Q^{\prime}(t)\right\rangle$ of the action (2) yields

$$
\begin{aligned}
0= & \int_{t_{1}}^{t_{2}} \mathrm{~d} t\left\langle Q^{\prime}(t)\left|\exp \{-i \delta \varepsilon(t) \underset{\sim}{G}\}\left(i \frac{d}{d t}-\underset{\sim}{H}\right) \exp \{i \delta \varepsilon(t) \underset{\sim}{G}\}\right| Q^{\prime}(t)\right\rangle \\
= & \int_{t_{1}}^{t_{2}} \mathrm{~d} t \delta \varepsilon(t)\left\{\frac{d}{d t}\left\langle Q^{\prime}(t)|\underset{\sim}{G}| Q^{\prime}(t)\right\rangle-\left\langle Q^{\prime}(t)|i[\underset{\sim}{H}, \underset{\sim}{G}]| Q^{\prime}(t)\right\rangle\right\} \\
& + \text { total time derivative }+\operatorname{order}\left(\delta \varepsilon^{2}\right) .
\end{aligned}
$$

As $\delta \varepsilon(t)$ is arbitrary and vanishes at the end points one obtaines

$$
\begin{aligned}
\frac{d}{d t} \mathcal{G} & =\frac{d}{d t}\left\langle Q^{\prime}(t)|\underset{\sim}{G}| Q^{\prime}(t)\right\rangle \\
& =\{\mathcal{H}, \mathcal{G}\}=\left\langle Q^{\prime}(t)|i[\underset{\sim}{H}, \underset{\sim}{G}]| Q^{\prime}(t)\right\rangle .
\end{aligned}
$$

That means that for this class of generators the generalized Poisson bracket is just the expectation value of the commutator with $\underset{\sim}{\underset{\sim}{H}}$.

Relation (37) is very useful for two reasons. First, if $\underset{\sim}{G}$ commutes with the hamiltonian $\underset{\sim}{H}$ and $\exp (i \varepsilon \underset{\sim}{G})\left|Q_{1}^{\prime}\right\rangle=\left|Q_{2}^{\prime}\right\rangle$ then $\left\langle Q^{\prime}(t)|\underset{\sim}{G}| Q^{\prime}(t)\right\rangle$ is automatically a constant of motion. Second, this relation is an important guidance for the choice of the trial state $\left|Q^{\prime}\right\rangle$. If one wants the model to obey certain conservation laws then the set of trial states should be invariant under unitary transformations generated by the constants of motion. For example total momentum conservation implies that a translated trial state is again a valid trial state. Conservation of total spin $\underset{\sim}{\vec{J}}=\underset{\sim}{\vec{L}}+\underset{\sim}{\vec{S}}$ is guaranteed when a rotation of the trial state in coordinate and spin space results again in a trial state.

Relation (37) also sheds some light on the quality of the variational principle. It says that under the premises that $\exp (i \varepsilon \underset{\sim}{G})$ does not map out of the set of trial states the expectation value $\mathcal{G}(t)$ of $\underset{\sim}{G}$ develops for short times like the exact solution. From Eq. (37) follows that along the trajectory $\left|Q^{\prime}(t)\right\rangle$ the time derivative of $\mathcal{G}(t)$ equals the exact one. 


\section{B. From quantum to classical mechanics}

This section demonstrates that the time-dependent variational principle, discussed in general in the previous section, represents a method to go in a smooth way from quantum physics to classical physics by appropriately choosing the dynamical degrees of freedom in the trial state. In contrast to Ehrenfest's theorem this method also works for identical particles and in finite dimensional spin-spaces.

\section{Quantum mechanics}

As a first illustration let us represent the trial state in terms of an orthonormal basis $|n\rangle$ in many-body space. We may write a general state $\left|Q^{\prime}\right\rangle$ as

$$
\left|Q^{\prime}\right\rangle=\left|\rho_{1}, \rho_{2}, \cdots, \pi_{1}, \pi_{2}, \cdots\right\rangle=\sum_{n} \frac{1}{\sqrt{2}}\left(\rho_{n}+i \pi_{n}\right)|n\rangle \equiv \sum_{n} c_{n}|n\rangle
$$

where the complex amplitudes are written in terms of their real and imaginary parts $\rho_{n}$ and

$\pi_{n}$. It is easy to verify that the Lagrange function for normalizable states defined in Eq. (3) is given by

$$
\mathcal{L}^{\prime}=\sum_{n} \frac{1}{2}\left(\pi_{n} \dot{\rho}_{n}-\rho_{n} \dot{\pi}_{n}\right)+\frac{d}{d t} \sum_{n} \frac{i}{4}\left(\rho_{n}^{2}+\pi_{n}^{2}\right)-\mathcal{H}\left(\rho_{1}, \rho_{2}, \cdots, \pi_{1}, \pi_{2}, \cdots\right)
$$

The real Hamilton function, expressed with the real and imaginary parts of the matrix elements $H_{n k} \equiv\langle n|\underset{\sim}{H}| k\rangle$, is bilinear in $\rho$ and $\pi$

$$
\mathcal{H}\left(\rho_{1}, \rho_{2}, \cdots, \pi_{1}, \pi_{2}, \cdots\right)=\frac{1}{2} \sum_{k, n}\left[\left(\rho_{k} \rho_{n}+\pi_{k} \pi_{n}\right) \operatorname{Re} H_{k n}+\left(\pi_{k} \rho_{n}-\rho_{k} \pi_{n}\right) \operatorname{Im} H_{k n}\right]
$$

The Euler Lagrange equations

$$
\frac{d}{d t} \frac{\partial \mathcal{L}^{\prime}}{\partial \dot{\rho}_{n}}=\frac{\partial \mathcal{L}^{\prime}}{\partial \rho_{n}} \quad \text { and } \quad \frac{d}{d t} \frac{\partial \mathcal{L}^{\prime}}{\partial \dot{\pi}_{n}}=\frac{\partial \mathcal{L}^{\prime}}{\partial \pi_{n}}
$$

yield

$$
\frac{d}{d t} \pi_{n}=-\frac{\partial \mathcal{H}}{\partial \rho_{n}} \quad \text { and } \quad \frac{d}{d t} \rho_{n}=\frac{\partial \mathcal{H}}{\partial \pi_{n}} .
$$


There is a very important message to be learned from this little exercise: Eqs. (42) look exactly like Hamilton's equations of motion in which $\left(\rho_{n}, \pi_{n}\right)$ are pairs of canonical variables and $\mathcal{H}\left(\rho_{1}, \rho_{2}, \cdots, \pi_{1} \pi_{2}, \cdots\right)$ is the Hamilton function, bilinear in the coordinates and momenta of the system. But these seemingly classical equations are just a representation of the Schrödinger equation as can easily been seen by rewriting the two real Eqs. (42) in terms of the complex coefficients $c_{n}=\frac{1}{\sqrt{2}}\left(\rho_{n}+i \pi_{n}\right)$ as one complex equation, namely

$$
i \frac{d}{d t} c_{n}=\frac{\partial \mathcal{H}}{\partial c_{n}^{*}}=\sum_{k} H_{n k} c_{k} \quad \text { or } \quad i \frac{d}{d t}\left|Q^{\prime}\right\rangle=\underset{\sim}{H}\left|Q^{\prime}\right\rangle
$$

The mere fact that the equations of motion (42) appear in a classical form does not necessarily imply that the system is classical and for example violates the uncertainty relation or, in case of indistinguishable fermions, Fermi-Dirac statistics. This will be discussed in detail in Sec. V.

The symplectic structure of Eqs. (42) is fundamental to all energy conserving dynamical theories, classical, quantum or quantum field theories, see e.g. (Katz, 1965). Only the physical meaning of the dynamical variables $\rho_{n}$ and $\pi_{n}$ and of the Hamilton function $\mathcal{H}$ determine which kind of physical system one is dealing with.

In the example (38) $\rho_{n}$ and $\pi_{n}$ are not the $6 N$ positions and momenta of the $N$ particles but infinitely many variables which specify the many-body state $\left|Q^{\prime}\right\rangle$. A truncation to a finite number results in an approximation of the exact time evolution. The quality depends on how well the selected set of basis states, $\{|n\rangle\}$, can represent the part of the Hilbert space which is occupied by the physical system under consideration.

\section{Classical mechanics}

A second example shows (Heller, 1975) that one gets the classical Hamilton equations of motion for the $6 N$ positions and momenta from the time-dependent variational principle by choosing the following dynamical variables. The normalized trial state $|Q\rangle$ is set up to describe $N$ distinguishable particles which are localized in phase space. 


$$
|Q\rangle=\left|\vec{r}_{1}, \vec{p}_{1}\right\rangle \otimes\left|\vec{r}_{2}, \vec{p}_{2}\right\rangle \otimes \cdots \otimes\left|\vec{r}_{N}, \vec{p}_{N}\right\rangle
$$

The single-particle states $\left|\vec{r}_{k}, \vec{p}_{k}\right\rangle$ in the direct product are taken to be the closest quantum analogue to classical particles, namely gaussian wave packets of minimum uncertainty, i.e. coherent states (Klauder and Skagerstam, 1985):

$$
\begin{aligned}
& \left\langle\vec{x} \mid \vec{r}_{k}, \vec{p}_{k}\right\rangle=\left(\frac{1}{\pi a_{0}}\right)^{3 / 4} \exp \left\{-\frac{\left(\vec{x}-\vec{r}_{k}\right)^{2}}{2 a_{0}}+i \vec{p}_{k} \vec{x}\right\} \\
& \left\langle\vec{k} \mid \vec{r}_{k}, \vec{p}_{k}\right\rangle=\left(\frac{a_{0}}{\pi}\right)^{3 / 4} \exp \left\{-\frac{a_{0}\left(\vec{k}-\vec{p}_{k}\right)^{2}}{2}-i \vec{r}_{k}\left(\vec{k}-\vec{p}_{k}\right)\right\} .
\end{aligned}
$$

The dynamical variables $\vec{r}_{k}$ and $\vec{p}_{k}$ are just the mean values of the position and momentum operator, respectively

$$
\vec{r}_{k}=\left\langle\vec{r}_{k}, \vec{p}_{k}|\underset{\sim}{\vec{x}}| \vec{r}_{k}, \vec{p}_{k}\right\rangle, \quad \vec{p}_{k}=\left\langle\vec{r}_{k}, \vec{p}_{k}|\underset{\sim}{\vec{k}}| \vec{r}_{k}, \vec{p}_{k}\right\rangle
$$

The width parameter $a_{0}$ is a real fixed number here. In later applications it will also be taken as a complex dynamical variable.

The evaluation of the Lagrange function (14) is again simple and yields

$$
\mathcal{L}=-\sum_{k=1}^{N} \vec{r}_{k} \dot{\vec{p}}_{k}-\mathcal{H}
$$

where the Hamilton function $\mathcal{H}=\langle Q|\underset{\sim}{\underset{\sim}{H}}| Q\rangle$

$$
\mathcal{H}=\sum_{k=1}^{N}\left(\frac{\vec{p}_{k}^{2}}{2 m_{k}}+\frac{3}{4 m_{k} a}\right)+\sum_{k<l=1}^{N}\left\langle\vec{r}_{k}, \vec{p}_{k}\left|\otimes\left\langle\vec{r}_{l}, \vec{p}_{l}|\underset{\sim}{V}(1,2)| \vec{r}_{k}, \vec{p}_{k}\right\rangle \otimes\right| \vec{r}_{l}, \vec{p}_{l}\right\rangle
$$

is the expectation value of the hamiltonian

$$
\underset{\sim}{H}=\sum_{l=1}^{N} \underset{\stackrel{\vec{k}^{2}}{\stackrel{\vec{c}^{2}}{2}(l)}}{2 m_{l}}+\sum_{k<l=1}^{N} \underset{\sim}{V}(k, l) .
$$

The Euler Lagrange equations

$$
\begin{aligned}
& \frac{d}{d t} \frac{\partial \mathcal{L}}{\partial \dot{\vec{r}}_{k}}=\frac{\partial \mathcal{L}}{\partial \vec{r}_{k}} \quad \rightarrow \quad 0=-\dot{\vec{p}}_{k}-\frac{\partial \mathcal{H}}{\partial \vec{r}_{k}} \\
& \frac{d}{d t} \frac{\partial \mathcal{L}}{\partial \dot{\vec{p}}_{k}}=\frac{\partial \mathcal{L}}{\partial \vec{p}_{k}} \quad \rightarrow \quad-\dot{\vec{r}}_{k}=-\frac{\partial \mathcal{H}}{\partial \vec{p}_{k}}
\end{aligned}
$$

result in 


$$
\frac{d}{d t} \vec{p}_{k}=-\frac{\partial \mathcal{H}}{\partial \vec{r}_{k}} \quad \text { and } \quad \frac{d}{d t} \vec{r}_{k}=\frac{\partial \mathcal{H}}{\partial \vec{p}_{k}}
$$

In a situation where classical mechanics holds, i.e. the wave packets are narrow enough so that one can approximate the expectation value

$$
\langle Q|\underset{\sim}{H}| Q\rangle=\mathcal{H}\left(\vec{r}_{1}, \vec{r}_{2}, \cdots, \vec{p}_{1}, \vec{p}_{2}, \cdots\right) \approx H\left(\vec{r}_{1}, \vec{r}_{2}, \cdots, \vec{p}_{1}, \vec{p}_{2}, \cdots\right)
$$

by replacing the momentum and position operators in the hamiltonian by their respective mean values of the wave packets, Eqs. (53) become the classical Hamilton's equations of motion.

Up to this point the time-dependent variational principle leads to the same results as Ehrenfest's theorem which usually establishes the connection between quantum and classical systems. As will be demonstrated in section III the prescription to replace the operators in the Heisenberg equation by their mean values does not work for indistinguishable particles. But the time-dependent variational principle for an antisymmetrized trial state $|Q\rangle$ provides the molecular dynamics equations for identical fermions. For bosons one would of course use a symmetrized state.

The canonical pair $\left(\vec{r}_{k}, \vec{p}_{k}\right)$ of real position and momentum can be combined to a complex variable $z_{k}=\sqrt{\frac{1}{2 a_{0}}} \vec{r}_{k}+i \sqrt{\frac{a_{0}}{2}} \vec{p}_{k}$. The Hamilton equations (53) written in terms of $z_{k}$ and $z_{k}^{*}$ take the form

$$
i \frac{d}{d t} z_{k}=\frac{\partial}{\partial z_{k}^{*}} \mathcal{H}\left(z_{1}^{*}, z_{2}^{*}, \cdots, z_{1}, z_{2}, \cdots\right)
$$

which formally looks like the Schrödinger equation (43).

This and the previous example show that one cannot decide from the form of the equations of motion alone whether the system, they are describing, is classical or quantal. Furthermore, the time-dependent variational principle can provide both, classical and quantal many-body equations of motion, depending how the trial state $|Q\rangle$ is chosen. In the following third example an intermediate situation is sketched, where some quantum effects are included. 
A third example which has part of quantum effects included is a trial state in which the width parameter $a$ is a dynamical variable and complex, $a=a_{R}+i a_{I}$ (Tsue and Fujiwara, 1991). Take the $N$-body trial state to be a product state

$$
|Q\rangle=\left|q_{1}\right\rangle \otimes\left|q_{2}\right\rangle \otimes \cdots \otimes\left|q_{N}\right\rangle
$$

of single-particle states, which are gaussians

$$
\left\langle\vec{x} \mid q_{l}\right\rangle=\left\langle\vec{x} \mid \vec{r}_{l}, \vec{p}_{l}, a_{l}\right\rangle=\left(2 \pi \frac{a_{l}^{*} a_{l}}{a_{l}^{*}+a_{l}}\right)^{-3 / 4} \exp \left\{-\frac{\left(\vec{x}-\vec{r}_{l}\right)^{2}}{2 a_{l}}+i \vec{p}_{l} \vec{x}+i \phi_{l}\right\}
$$

characterized by their mean positions $\vec{r}_{l}$, mean momenta $\vec{p}_{l}$, widths $a_{l}$ and phases $\phi_{l}$.

For a single-particle hamiltonian which contains in addition to the kinetic energy a harmonic oscillator potential

$$
\underset{\sim}{H_{\mathrm{HO}}}=\sum_{l=1}^{N} \underset{\mathrm{h}}{\stackrel{h}{\sim}} \mathrm{HO}(l)=\sum_{l=1}^{N}\left(\underset{\frac{\vec{k}^{2}}{\stackrel{\sim}{2} m_{l}}}{2 l)}+\frac{1}{2} m_{l} \omega^{2} \underset{\sim}{\vec{x}^{2}(l)}\right) .
$$

the equations of motion are

$$
\begin{aligned}
& \frac{d}{d t} \vec{r}_{l}=\frac{\vec{p}_{l}}{m_{l}}, \quad \frac{d}{d t} \vec{p}_{l}=-m_{l} \omega^{2} \vec{r}_{l} \\
& \frac{d}{d t} a_{l}=\frac{i}{m_{l}}-i m_{l} \omega^{2} a_{l}^{2}, \quad \frac{d}{d t} \phi_{l}=-\frac{\vec{p}_{l}^{2}}{2 m_{l}}-\frac{3 a_{R l}}{2 m_{l}\left|a_{l}\right|^{2}}-\frac{m_{l}}{2} \omega^{2} \vec{r}_{l}^{2} .
\end{aligned}
$$

These equations, although looking classically for $\vec{r}_{l}$ and $\vec{p}_{l}$, represent the exact solution of the Schrödinger equation, provided the wave function is a gaussian wave packet at time zero. The centers of the wave packets as well as the mean momenta oscillate harmonically with the frequency $\omega$. Due to the time dependence of the widths the packets also breath but with twice the frequency. The solution is fully quantum mechanical, although described by only a few (classically looking) parameters. Free motion without a potential $(\omega=0)$ is of course also exact. For general potentials the trial state (57) may serve as an approximation if locally, in the region where $\left\langle\vec{x} \mid q_{l}\right\rangle$ does not vanish, the potential is well represented by a harmonic oscillator. 
In general one can say, that a trial state provides the exact solution of the Schrödinger equation if the action of the Hamilton operator $\underset{\sim}{H}$ on the trial state can be expressed in terms of parameters and first derivatives with respect to the parameters like in the following example

$$
\underset{\sim}{\vec{k}}|q\rangle=\left(i \frac{\partial}{\partial \vec{r}}-i \vec{p} \frac{\partial}{\partial \phi}\right)|q\rangle
$$

and

$$
\underset{\sim}{\vec{k}^{2}}|q\rangle=\left(2 i \frac{\partial}{\partial a_{I}}+2 i \vec{p} \cdot \frac{\partial}{\partial \vec{r}}-i\left[\vec{p}^{2}+3 \frac{a_{R}}{a_{R}^{2}+a_{I}^{2}}\right] \frac{\partial}{\partial \phi}\right)|q\rangle .
$$

For the harmonic oscillator the Schrödinger equation takes the form (index $l$ omitted)

$$
\begin{aligned}
i \frac{d}{d t}|q\rangle= & i \sum_{\nu} \dot{q}_{\nu} \frac{\partial}{\partial q_{\nu}}|q\rangle=\underset{\sim}{h} \mathrm{HO}|q\rangle \\
= & \left\{i\left(\frac{\vec{p}}{m}\right) \frac{\partial}{\partial \vec{r}}-i\left(m \omega^{2} \vec{r}\right) \frac{\partial}{\partial \vec{p}}\right. \\
& +i\left(2 m \omega^{2} a_{R} a_{I}\right) \frac{\partial}{\partial a_{R}}+i\left(\frac{1}{m}-m \omega^{2}\left(a_{R}^{2}-a_{I}^{2}\right)\right) \frac{\partial}{\partial a_{I}} \\
& \left.\quad-i\left(\frac{\vec{p}^{2}}{2 m}+\frac{3 a_{R}}{2 m|a|^{2}}-\frac{m}{2} \omega^{2} \vec{r}^{2}\right) \frac{\partial}{\partial \phi}\right\}|q\rangle .
\end{aligned}
$$

From Eq. (63) the equations of motion (59) and (60) follow at once. Because gaussian wave packets are an exact solution of the Schrödinger equation for these one-body hamiltonians, also the respective product states are an exact solution for the corresponding many-body problem. Moreover, since antisymmetrization and symmetrization commute with the exact time evolution, the equations of motion (59) remain the same for antisymmetric product states (identical fermions) and symmetric product states (identical bosons).

The time-dependent width parameter $a$, which describes the variances in coordinate and momentum space, provides the first nonclassical degree of freedom in the parameter manifold. It completes the classical equations (59) to the full quantum solution for spherical harmonic oscillator potentials as well as for free motion. In section $\mathrm{V}$ it is shown that also in thermodynamic considerations the inclusion of finite widths leads to quantum statistics. 


\section{Further remarks}

In the previous Sec. II B several examples are presented to get acquainted with the numerous aspects of the time-dependent variational principle and its function as a bridge between quantum and classical physics. We add now further remarks which are again of general type and useful to understand the different models referred to later.

\section{Selfconsistency and nonlinearity}

The equations of motion (11) (or (22)) determine the time evolution of the parameters $q_{\nu}(t)$ in parameter space. But, even if these parameters have an intuitive physical meaning, they first of all determine the trial state $\left|Q^{\prime}(t)\right\rangle$ from which all physical observables have to be calculated in a quantum fashion. Therefore, we derive here the corresponding equation of motion for $\left|Q^{\prime}(t)\right\rangle$ in Hilbert space.

The Hamilton operator $\underset{\sim}{H}$, which evolves the trial state $\left|Q^{\prime}(t)\right\rangle$ in time according to the equations of motion (11), has to fulfill

$$
\underset{\sim}{H_{0}}\left|Q^{\prime}\right\rangle=i\left|\dot{Q}^{\prime}\right\rangle \equiv i \sum_{\nu} \dot{q}_{\nu} \frac{\partial}{\partial q_{\nu}}\left|Q^{\prime}\right\rangle
$$

It is given by

$$
\underset{\sim}{H_{0}}=i\left(\left|\dot{Q}^{\prime}\right\rangle\left\langle Q^{\prime}|-| Q^{\prime}\right\rangle\left\langle\dot{Q}^{\prime}\right|\right)+\frac{i}{2}\left|Q^{\prime}\right\rangle\left(\left\langle\dot{Q}^{\prime} \mid Q^{\prime}\right\rangle-\left\langle Q^{\prime} \mid \dot{Q}^{\prime}\right\rangle\right)\left\langle Q^{\prime}\right|
$$

where $\left|\dot{Q}^{\prime}\right\rangle$ stands for

$$
\left|\dot{Q}^{\prime}\right\rangle=\frac{d}{d t}\left|Q^{\prime}\right\rangle=-i \sum_{\mu, \nu} \frac{\partial}{\partial q_{\mu}}\left|Q^{\prime}\right\rangle \mathcal{C}_{\mu \nu}^{\prime-1} \frac{\partial \mathcal{H}}{\partial q_{\nu}^{*}}
$$

and analogue for $\left\langle\dot{Q}^{\prime}\right|$. Thus $\underset{\sim}{H}$. itself depends on $Q^{\prime *}$ and $Q^{\prime}$. Using the fact that $\left|Q^{\prime}\right\rangle$ is always normalized it is easy to show that $\underset{\sim}{H_{0}}$ defined in $(65)$ is the generator of the approximate time evolution

$$
i \frac{d}{d t}\left|Q^{\prime}\right\rangle=\underset{\sim}{H_{0}}\left(Q^{\prime *}, Q^{\prime}\right)\left|Q^{\prime}\right\rangle
$$


The equation of motion (67) for the trial state in Hilbert space is the counterpart for the equation of motion (11) in parameter space

$$
i \dot{q}_{\mu}=\sum_{\nu} \mathcal{C}_{\mu \nu}^{\prime-1}\left(Q^{\prime *}, Q^{\prime}\right) \frac{\partial}{\partial q_{\nu}^{*}} \mathcal{H}\left(Q^{\prime *}, Q^{\prime}\right) .
$$

Eq. (67) is selfconsistent in the sense that the hamiltonian depends on the actual state $\left|Q^{\prime}(t)\right\rangle$. For example, if $\left|Q^{\prime}(t)\right\rangle$ is a single Slater determinant, but otherwise unrestricted, $\underset{\sim}{H}{ }_{0}\left(Q^{\prime *}, Q^{\prime}\right)$ is the Hartree-Fock hamiltonian (Kerman and Koonin, 1976). Furthermore, the approximate Eq. (67) is usually not a linear equation like the exact Schrödinger equation and therefore violates the superposition principle of quantum mechanics.

Both, selfconsistency and nonlinearity are also common to classical molecular dynamics where the classical Hamilton function $H\left(r_{1}(t), \cdots, p_{1}(t), \cdots\right)$ depends on the actual physical state. The nonlinearity of $H\left(r_{1}(t), \cdots, p_{1}(t), \cdots\right)$ plays an important rôle for statistical properties like equilibration and chaotic behaviour, see Sec. V. In quantum mechanics these properties can only be investigated by breaking the superposition principle by coarse graining or phase averaging.

\section{Quantum branching}

The time-dependent variational principle creates an approximate quantum dynamics in a manifold of trial states. Due to the severe restrictions it is very likely that areas of the Hilbert space are not reachable by the approximate equations of motion which would be visited by the solution of the Schrödinger equation. The idea for an improved model is to allow branching from one trajectory $\left|Q_{i}^{\prime}(t)\right\rangle$ to another one $\left|Q_{j}^{\prime}(t)\right\rangle$, i.e. to jump in the parameter manifold with a certain probability.

If the local deviation between the exact solution and the solution of the time-dependent variational principle, see Sec. II A 2, is not so large, the operator

$$
\begin{aligned}
\Delta \underset{\sim}{H} & =\left(\underset{\sim}{H}-i \sum_{\nu} \dot{q}_{\nu} \frac{\partial}{\partial q_{\nu}}\right) \\
& \equiv \underset{\sim}{H}-{\underset{\sim}{H}}_{0}
\end{aligned}
$$


which appears in Eq. (24) for the error, may be regarded as a perturbation, which causes transitions between the trial states $\left|Q_{i}^{\prime}(t)\right\rangle$, while each $\left|Q_{i}^{\prime}(t)\right\rangle$ follows the approximate time evolution as given in Eqs. (65) and (66) with its selfconsistent hamiltonian $\underset{\sim}{H_{0}}\left(Q_{i}^{\prime *}, Q_{i}^{\prime}\right)$. As in usual pertubation theory the transition $\left|Q_{i}^{\prime}(t)\right\rangle \rightarrow\left|Q_{j}^{\prime}(t)\right\rangle$ should be related to the amplitude $\left\langle Q_{j}^{\prime}(t)|\underset{\sim}{\Delta}| Q_{i}^{\prime}(t)\right\rangle$ (Tully, 1990; Topaler et al., 1997; Lacroix, Chomaz, and Ayik, 1998, 1999). One would, however, like to work with probabilities instead of amplitudes. This should be possible if the physical system is in an energy regime with high level density where statistical arguments may be used.

In literature one often employs phenomenological arguments (see Sec. IV) for random jumps in parameter space. The above sketched procedure which refers to $\underset{\sim}{\sim} \underset{\sim}{\sim}$ has several advantages. The most important is selfconsistency, the transition depends on the actual situation and the choice of the trial states $\left|Q_{i}^{\prime}(t)\right\rangle$. If for example $\left|Q_{i}^{\prime}(t)\right\rangle$ is already a solution of the Schrödinger equation, $\underset{\sim}{H}\left|Q_{i}^{\prime}(t)\right\rangle=0$, no branching occures.

Another important aspect is the breaking of symmetries (Colonna and Chomaz, 1998). Whereas the exact quantum state can retain dynamically conserved symmetries by linear superpositions of states, the nonlinearity and selfconsistency of the approximate hamiltonian (65) usually does not permit that. Take for example the mirror symmetry of a molecule or a nucleus which breaks into pieces. Each measured final channel which corresponds to one $\left|Q_{i}^{\prime}\right\rangle$ does not possess mirror symmetry but the ensemble of all measured channels does. If the initial trial state $\left|Q^{\prime}(t=0)\right\rangle$ has this symmetry and $\underset{\sim}{H}$ does not break it explicitly the symmetry will be kept in $\left|Q^{\prime}(t)\right\rangle$. Here, quantum branching into a nonsymmetric trial state $\left|Q_{j}^{\prime}\right\rangle$ and its mirrored counterpart $\left|-Q_{j}^{\prime}\right\rangle$ with equal probabilities would resolve the problem of spurious cross channel coupling observed in selfconsistent approximations (Griffin et al., 1980). The transitions may also allow quantum fluctuations to configurations which are close in Hilbert space but cannot be reached by the approximate time evolution, for example tunneling.

Another noteworthy example of quantum branching is displayed in Fig. 2 which in a pictorial way shows four gaussian wave packets $\left|q_{l}(t)\right\rangle$ (see Eq. (57)) forming a bound 


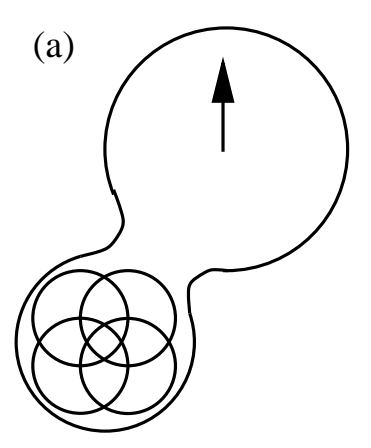

(b)
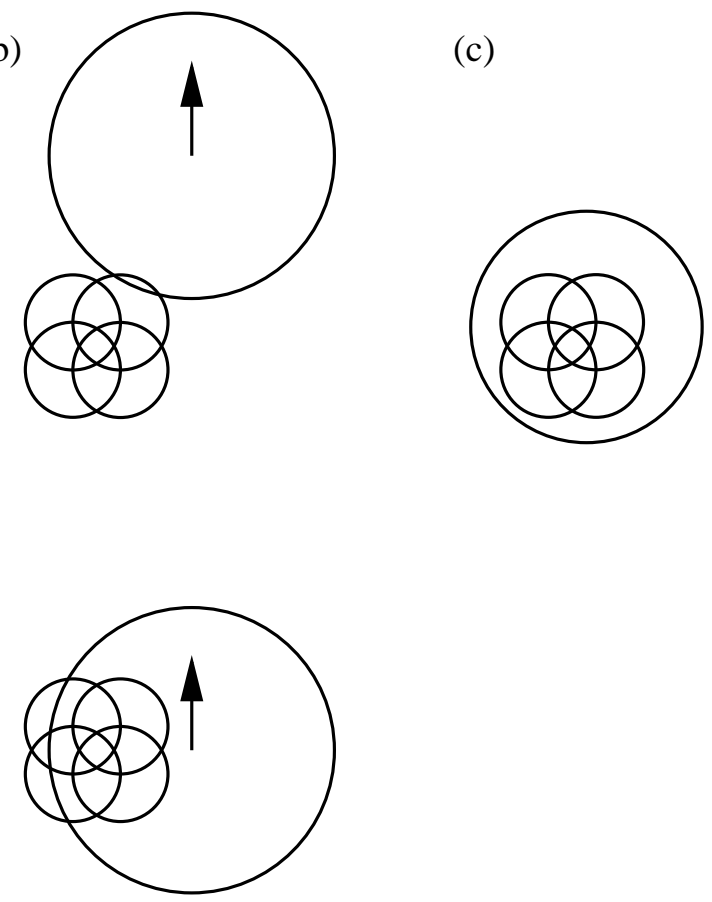

FIG. 2. Sketch of a four particle cluster and a passing wave packet. Circles indicate half-density contours. The molecular dynamics equations of motion for the centroids and the widths of the packets can only influence the wave packet as a whole, either it passes by (b) or it is captured (c). The exact solution allows to split the packet into a captured and proceeding part (a) (see text).

cluster and a wave packet (large circle) of an unbound particle which passes by. The timedependent width of this wave packet has spread in coordinate space because it was moving freely for some time. Such situations occurr frequently in simulations. In the exact case a piece of this wave packet would stick to the four particle cluster indicating that, with a certain probability, a five particle cluster has been created. The remaining part of the wave packet would move on. Because the gaussian single-particle state does not permit this freedom the wave packet can only be bound to the cluster in total or escape. However, in order to become bound its width has to shrink to the size of the cluster otherwise the matrix element of the interaction with the other wave packets is too weak. It is obvious that beyond a certain size of the passing packet the overlap with the cluster will be too small to create a 
sufficiently large generalized force on its width parameter to let it shrink. Therefore, small capture probabilities cannot be described.

A branching procedure would in the above given example divide the time-evolution into a branch of a five particle cluster and one of a four particle cluster and a freely moving wave packet with the respective probabilities. In general one can say whenever the interaction varies strongly across the region of the wave packet additional shape degrees of freedom should be considered. Otherwise local actions are washed out and only mean field properties survive.

But quantum branching faces also problems. In going from amplitudes to probabilities one should not violate conservation laws. For instance, one should not alter the energy distribution of the system. The mean value and the variance of the energy of the ensemble of trial states, which are populated during the branching should be the same as in the initial state. The same is true for other conserved quantities like total momentum or angular momentum. It is quite conceivable that variational principles with the appropriate constraints could be helpful (Balian and Veneroni, 1988; Lacroix, Chomaz, and Ayik, 1998, 1999).

\section{Approximation or new dynamical model?}

At this point some philosophical thoughts on the meaning of the results from the timedependent variational principle seem to be appropriate. One could of course always claim

that any restriction of the degrees of freedom in the trial state $\left|Q^{\prime}\right\rangle$ leads to approximations of the Schrödinger equation, the more restraint the trial state the worse the approximation. But the simple examples in Sec. II B and those for antisymmetric states in the following Sec. III demand a more differentiated point of view.

Let us exemplify this by restricting step by step the degrees of freedom in the general quantum state for a number of atoms. First we make a restricted ansatz for the trial state which contains only the coordinates and spins of nucleons and electrons and we disregard completely the internal quark and gluon degrees of freedom for the nucleons and the quanta of 
the electromagnetic field. For low excitation energies this is certainly a good approximation.

The next step is to neglect the internal degrees of freedom of the nuclei assuming that they are all in their ground states and to retain only their center of mass coordinates and the electron variables. But this is still too complex to solve the Schrödinger equation. Therefore we describe the c.m. motion of the heavy nuclei by gaussian packets, which leads to the classical equations of motion (53) for the nuclei with a Hamilton function $\mathcal{H}$ which couples to the quantal electrons. The state for the electrons may then be constrained to a single Slater determinant with no further restrictions on the single-particle states. Now we have arrived at the time-dependent Hartree Fock model which is a mean-field theory. The selfconsistent hamiltonian $\underset{\sim}{H_{0}}$ is a one-body operator.

But we can of course go further and disregard the internal degrees of freedom of the atoms or the molecules altogether and treat only their center of mass motion by means of fixed-width gaussians. At this point we obtain classical molecular dynamics with a Hamilton function $\mathcal{H}$ which contains two-body potentials between the molecules.

If the molecules are big, may be junks of crystals, may be even stars hold together by gravity, their center of mass coordinates may be the adequate parameters for very narrow gaussians. The time-dependent variational principle will now provide Newton's equations for macroscopic objects.

We don't believe that Newtons equations should be viewed as a bad approximation (bad because of the many constraints) of Quantum-Chromo-Dynamics where we started. ${ }^{3}$ They should also not be regarded as an approximation to time-dependent Hartree Fock, which formally one could plead for, because further constraints on the Slater determinant led there.

The question is rather, which are the relevant degrees of freedom for a physical system. When those are identified the time-dependent variational principle provides a dynamical

\footnotetext{
${ }^{3}$ The time-dependent variational principle can also be formulated in quantum field theory and it reduces to the nonrelativistic one discussed here.
} 
model which is selfconsistent and has all properties of a Lagrange formalism, like existence of canonical variables, Noether's theorem, etc.

Since the restriction of the trial state $\left|Q^{\prime}\right\rangle$ can be made in finer or coarser steps it is a matter of debate at which point one wants to speak about a new dynamical model and when of an approximation. 


\section{ANTISYMMETRIZATION}

The previous chapter demonstrated how to derive classical equations of motion from quantum mechanics by means of the time-dependent variational principle and localized single-particle states. It was also shown that there is no clear boundary between quantum and classical mechanics. This will be even more the case in this chapter where we are dealing with indistinguishable fermions.

The natural generalization of the product state (44) (which led to classical molecular dynamics) to a trial state for identical fermions is the antisymmetrized product

$$
\begin{aligned}
|Q\rangle & =\underset{\sim}{A}\left|q_{1}\right\rangle \otimes\left|q_{2}\right\rangle \otimes \cdots \otimes\left|q_{N}\right\rangle \\
& =\frac{1}{N !} \sum_{\text {all } P} \operatorname{sgn}(P)\left|q_{P(1)}\right\rangle \otimes\left|q_{P(2)}\right\rangle \otimes \cdots \otimes\left|q_{P(N)}\right\rangle .
\end{aligned}
$$

The sum runs over all permutations $P$ and $\operatorname{sgn}(P)$ is the sign of the permutation. The localized single-particle states $\left|q_{l}\right\rangle$ contain for spin- $\frac{1}{2}$ particles also two-component spinors $\left|\chi_{l}\right\rangle$

$$
\left\langle\vec{x} \mid q_{l}\right\rangle=\exp \left\{-\frac{\left(\vec{x}-\vec{b}_{l}\right)^{2}}{2 a_{l}}\right\} \otimes\left|\chi_{l}\right\rangle, \quad \vec{b}_{l}=\vec{r}_{l}+i a_{l} \vec{p}_{l}
$$

The spinor may for example be represented by the two complex parameters $\chi_{l}^{\uparrow}=\left\langle\uparrow \mid \chi_{l}\right\rangle$ and $\chi_{l}^{\downarrow}=\left\langle\downarrow \mid \chi_{l}\right\rangle$, where $|\uparrow\rangle$ and $|\downarrow\rangle$ are the eigenstates of the spin operator $\underset{\sim}{\underset{s}{z}}$.

The general Lagrangian $\mathcal{L}$, as given in Eq. (14), calculated with the antisymmetrized trial state (70) provides through the Euler Lagrange equations (21) or (22) the desired molecular dynamics equations for fermions

$$
i \sum_{\nu} \mathcal{C}_{\mu \nu}\left(Q^{*}, Q\right) \dot{q}_{\nu}=\frac{\partial \mathcal{H}\left(Q^{*}, Q\right)}{\partial q_{\mu}^{*}}
$$

The hermitian matrix $\mathcal{C}_{\mu \nu}\left(Q^{*}, Q\right)$, Eq. (23), is not as simple as in the classical case, where $\mathcal{C}_{\mu \nu}=\delta_{\mu \nu}$, compare Eq. (55), but depends on all parameters contained in $Q$.

Since the trial state is antisymmetric all consequences of the Pauli principle are incorporated in the equations of motion $(72) \cdot \mathcal{C}_{\mu \nu}\left(Q^{*}, Q\right)$, which is the second logarithmic derivative 
of the determinant $\langle Q \mid Q\rangle=\operatorname{det}\left\{\left\langle q_{k} \mid q_{l}\right\rangle\right\}$, plays the rôle of a metric and will lead for example to large velocities $\dot{q}_{\nu}$ when the fermions get close to Pauli forbidden regions in phase space. In the energy $\mathcal{H}\left(Q^{*}, Q\right)=\langle Q|\underset{\sim}{\operatorname{H}}| Q\rangle /\langle Q \mid Q\rangle$ the Pauli principle causes exchange terms which induce for example additional momentum dependences. It should also be noted that the determinantal structure together with the nonorthogonality of the single-particle states result in an expression for the kinetic energy

$$
\frac{\langle Q|\underset{\sim}{T}| Q\rangle}{\langle Q \mid Q\rangle}=\sum_{k, l=1}^{N}\left\langle q_{k}|\underset{\sim}{t}| q_{l}\right\rangle \mathcal{O}_{l k}
$$

which contains a two-fold summation over all states (particles) compared to the single summation in classical molecular dynamics, Eq. (49). $\mathcal{O}_{l k}$ is the inverse of the overlap matrix

$$
\left(\mathcal{O}^{-1}\right)_{k l}:=\left\langle q_{k} \mid q_{l}\right\rangle ; \quad k, l=1, \cdots, N
$$

The expectation value of a two-body operator like the interaction is a four-fold sum

$$
\frac{\langle Q|\underset{\sim}{V}| Q\rangle}{\langle Q \mid Q\rangle}=\frac{1}{2} \sum_{k, l, m, n=1}^{N}\left\langle q_{k} q_{l}|\underset{\sim}{v}| q_{m} q_{n}\right\rangle\left(\mathcal{O}_{m k} \mathcal{O}_{n l}-\mathcal{O}_{m l} \mathcal{O}_{n k}\right) .
$$

The generalized forces $-\frac{\partial}{\partial q_{\mu}} \mathcal{H}\left(Q^{*}, Q\right)$ are therefore rather involved expressions which reflect the fact that the antisymmetrization $\underset{\sim}{A}$ is a $N$-body operation which correlates all $N$ particles simultaneously.

The apparent large numerical effort led different authors to propose approximations which will be discussed in Sec. IV.

First we explain in some length the two-body case because many of the new features concerning antisymmetrization and indistinguishability can be understood in this simple study. Furthermore, attempts to approximate the effects of the Pauli principle in molecular dynamics by so called Pauli potentials are based on considerations in two-body space.

The consequences of antisymmetrization in many-body space, which are discussed in Sec. III B, are even more intricate. Depending on how much the single-particle states overlap the antisymmetrization can change the properties of the trial state completely. There is for example Fermi motion even if the gaussian single-particle states have no mean momentum. 


\section{A. Effects of antisymmetrization in two-body space}

\section{Static considerations}

For two distinguishable particles the most simple trial state which leads in the proper limit to classical mechanics is the product of two gaussians $\left|q_{1}\right\rangle \otimes\left|q_{2}\right\rangle$. The corresponding state for indistinguishable fermions is the projection (70) onto the antisymmetric component

$$
|Q\rangle=\underset{\sim}{A}\left|q_{1}\right\rangle \otimes\left|q_{2}\right\rangle=\frac{1}{2 !}\left\{\left|q_{1}\right\rangle \otimes\left|q_{2}\right\rangle-\left|q_{2}\right\rangle \otimes\left|q_{1}\right\rangle\right\}
$$

In order to simplify the following discussion we do not use this Slater determinant but a trial state which separates center of mass and relative motion

$$
|Q\rangle=\left|q_{c m}\right\rangle \otimes|q\rangle
$$

The center-of-mass wave function is parameterized by $q_{c m}=\{A, \vec{B}\}$ as

$$
\left\langle\vec{X} \mid q_{c m}(t)\right\rangle=\exp \left\{-\frac{(\vec{X}-\vec{B}(t))^{2}}{2 A(t)}\right\}, \quad \vec{B}(t)=\vec{R}(t)+i A(t) \vec{P}(t),
$$

where $\vec{X}=\frac{1}{2}\left(\vec{x}_{1}+\vec{x}_{2}\right)$ is the center of mass coordinate and the parameter set $q_{c m}=\{A, \vec{B}\}$ contains the mean c.m. position $\vec{R}$ the mean c.m. momentum $\vec{P}$ combined in the complex parameter $\vec{B}$ and the complex width $A$.

The wave packet $|q\rangle$ for the relative motion contains also the spins, thus $q=$ $\left\{a, \vec{b}, \chi_{1}, \chi_{2}\right\}$. Its parametrized form in coordinate space reads

$$
\begin{aligned}
\langle\vec{x} \mid q(t)\rangle= & {\left[\exp \left\{-\frac{(\vec{x}-\vec{b}(t))^{2}}{2 a(t)}\right\}\left|\chi_{1}(t)\right\rangle \otimes\left|\chi_{2}(t)\right\rangle\right.} \\
& \left.-\exp \left\{-\frac{(\vec{x}+\vec{b}(t))^{2}}{2 a(t)}\right\}\left|\chi_{2}(t)\right\rangle \otimes\left|\chi_{1}(t)\right\rangle\right],
\end{aligned}
$$

where small letters denote the relative coordinates and parameters. In relative coordinate space the exchange of the two particles, $1 \leftrightarrow 2$, is equivalent to the parity operation $\vec{x}=$ $\vec{x}_{1}-\vec{x}_{2} \leftrightarrow-\vec{x}=\vec{x}_{2}-\vec{x}_{1}$.

The two-body wave packet (77) is in general not a single Slater determinant because the center of mass motion for a Slater determinant does not separate if the single-particle 
packets have different width parameters. In this section we choose the widths $A(t)$ for the center of mass motion and $a(t)$ for the relative packet to be independent in order to decouple the center-of-mass degree of freedom. It should also be noted that trial state (79) is a linear combination of total spin $S=0$ and $S=1$.

The relation between $\vec{b}$ and the more intuitive quantities "distance" $\vec{r}$ and "relative momentum" $\vec{p}$ is (time-dependence not indicated any longer)

$$
\begin{gathered}
\vec{b}_{R}=\vec{r}-a_{I} \vec{p} \quad \text { and } \quad \vec{b}_{I}=a_{R} \vec{p} \\
\vec{r}=\frac{a^{*} \vec{b}+a \vec{b}^{*}}{a+a^{*}} \quad \text { and } \quad \vec{p}=\frac{1}{i} \frac{\vec{b}-\vec{b}^{*}}{a+a^{*}}
\end{gathered}
$$

Here and throughout this section the real and imaginary part of complex numbers are denoted by the indices $R$ and $I$, respectively.

It is important to realize that $\vec{r}$ and $\vec{p}$ have their classical meaning only if the two particles are far away in phase space. We illustrate this effect of antisymmetrization in Fig. 3 where on the l.h.s. the relative wave function

$$
\left\langle\vec{x} \mid q_{d}\right\rangle=\left(2 \pi \frac{a^{*} a}{a^{*}+a}\right)^{-3 / 4} \exp \left\{-\frac{(\vec{x}-\vec{r})^{2}}{2 a}+i \vec{p} \vec{x}\right\}
$$

is plotted along the $\vec{r}$-direction. This trial state describes two distinguishable particles at relative distance $\vec{r}=\left\langle q_{d}|\underset{\sim}{\vec{x}}| q_{d}\right\rangle$. For a single gaussian the expectation value of $\underset{\sim}{\vec{x}}=$ $\underset{\sim}{\vec{x}}(1)-\underset{\sim}{\vec{x}}(2)$ is always equal to the parameter $\vec{r}$ independent of $\vec{p}$ and $a_{R}$.

This is a typical example where Ehrenfest's theorem can be used to derive the classical equations of motion from quantum mechanics. The method is to replace the expectation values of the Heisenberg equation

$$
\left\langle q_{d}\left|\frac{d}{d t} \vec{\sim}\right| q_{d}\right\rangle=\left\langle q_{d}\left|i\left[\frac{1}{2 \mu} \vec{\sim}^{2}, \underset{\sim}{\vec{x}}\right]\right| q_{d}\right\rangle=\frac{1}{\mu}\left\langle q_{d}|\underset{\sim}{\vec{k}}| q_{d}\right\rangle
$$

for narrow wave packets by the mean values

$$
\frac{d}{d t} \vec{r}=\frac{\vec{p}}{\mu}
$$

Analogue for the relative momentum operator $\underset{\sim}{\vec{k}}=\frac{1}{2}(\underset{\sim}{\vec{k}}(1)-\underset{\sim}{\vec{k}}(2))$ 

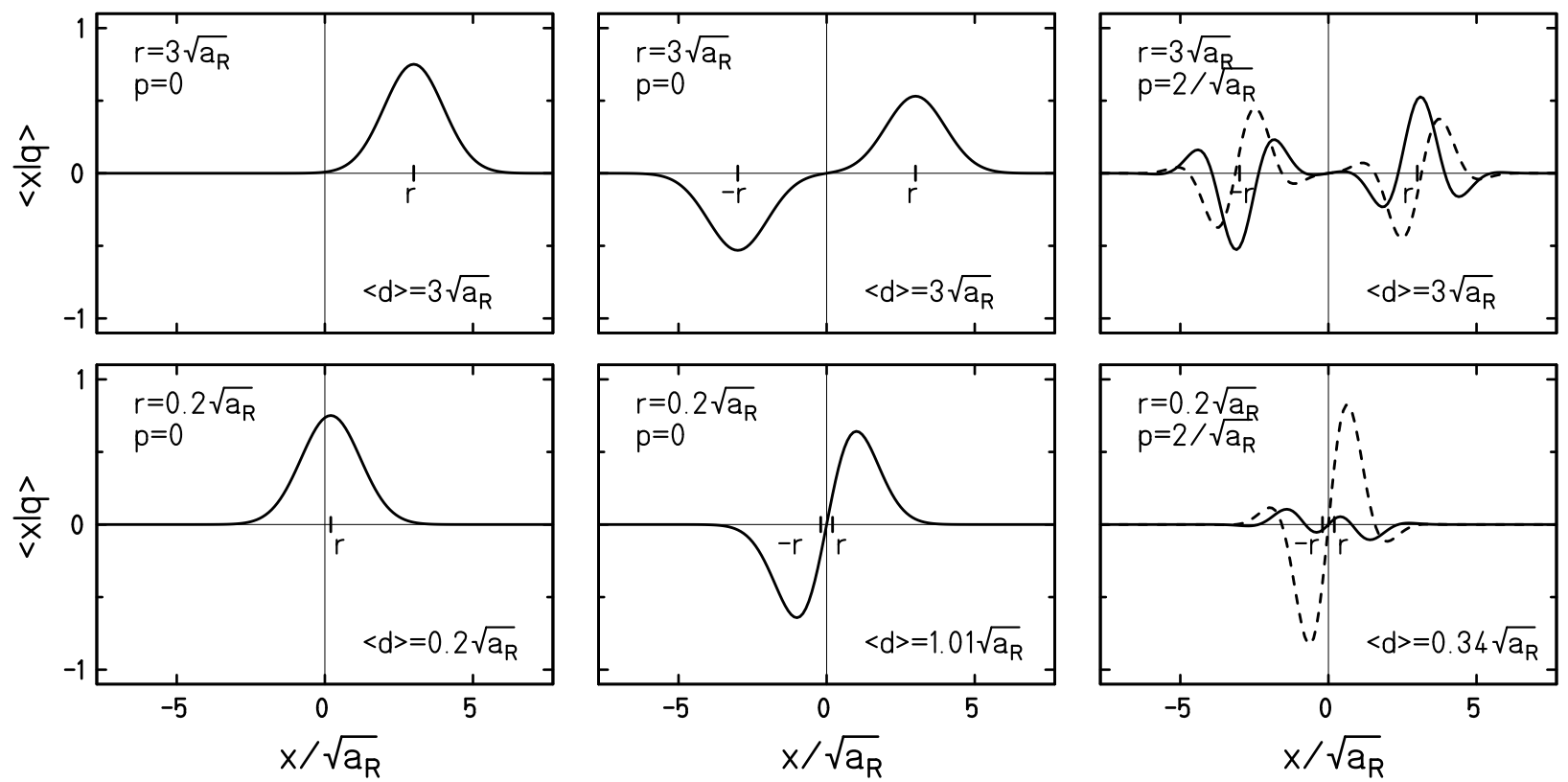

FIG. 3. Real (solid line) and imaginary part (dashed line) of the relative wave function for distinguishable particles (left column) and fermions (middle and right column) at different values of the parameters $\vec{r}=(r, 0,0), \vec{p}=(p, 0,0)$ and $a=a_{R}$. $\langle d\rangle$ measures the distance between particles, see Eq. (88).

$$
\left\langle q_{d}\left|\frac{d}{d t} \underset{\sim}{\vec{k}}\right| q_{d}\right\rangle=\left\langle q_{d}|i[V(\underset{\sim}{\vec{x}}), \underset{\sim}{\vec{k}}]| q_{d}\right\rangle=-\left\langle q_{d}\left|\frac{\partial}{\partial \vec{x}} V(\underset{\sim}{\vec{x}})\right| q_{d}\right\rangle
$$

or

$$
\frac{d}{d t} \vec{p} \approx \frac{\partial}{\partial \vec{r}} V(\vec{r})
$$

While (84) is exact for $\left|q_{d}\right\rangle,(86)$ is only an approximate expression which can be improved.

However, the antisymmetrized packet

$$
\langle\vec{x} \mid q\rangle=\left[\exp \left\{-\frac{(\vec{x}-\vec{r})^{2}}{2 a}+i \vec{p} \vec{x}\right\}-\exp \left\{-\frac{(\vec{x}+\vec{r})^{2}}{2 a}-i \vec{p} \vec{x}\right\}\right]
$$

displayed for two values of $\vec{r}$ and $\vec{p}$ in the middle and right column leads to different results. First, for indistinguishable particles the operator $\underset{\sim}{\vec{x}}=\underset{\sim}{\vec{x}}(1)-\underset{\sim}{\vec{x}}(2)$ is not an observable anymore, because it is not symmetric with respect to particle exchange. Due to its negative parity the expectation value $\langle q|\underset{\sim}{\vec{x}}| q\rangle /\langle q \mid q\rangle$ is always zero even for particles, which are far 
away from each other. The same holds true for the relative momentum, $\langle q|\underset{\sim}{\vec{\sim}}| q\rangle /\langle q \mid q\rangle=0$. Therefore Ehrenfest's theorem is changed into a triviality because Eqs. (83) and (85) become meaningless $(0=0)$. The conclusion is that Ehrenfest's theorem cannot be used to derive classical equations of motion for indistinguishable particles.

An observable which coincides with $|\vec{r}|$ at large distances is the root of the square radius minus the intrinsic width of the packet

$$
\langle d\rangle=\left(\frac{\left\langle q\left|\vec{\sim}^{2}\right| q\right\rangle}{\langle q \mid q\rangle}-\frac{3|a|^{2}}{2 a_{R}}\right)^{\frac{1}{2}} .
$$

In the middle of Fig. 3, which is for $\vec{p}=0$, it becomes evident that the observable distance $\langle d\rangle$ between two fermions with equal spin is larger than $|\vec{r}|$ and deviates most when $\vec{r}^{2} \ll a_{R}$. The r.h.s. with $|\vec{p}|=2 / \sqrt{a_{R}}$ demonstrates that the distance $\langle d\rangle$ depends also on the relative momentum. For large relative momenta, $\vec{p}^{2} a_{R} \gg 1$, the Pauli principle is again less effective and $\langle d\rangle \approx|\vec{r}|$. The explicit expression for the mean square radius in terms of parameters is

$$
\frac{\left\langle q\left|\underset{\sim}{\vec{x}^{2}}\right| q\right\rangle}{\langle q \mid q\rangle}=\vec{r}^{2}+\frac{3|a|^{2}}{2 a_{R}}+\frac{|a|^{2}}{a_{R}} f_{e x}\left(\xi, S_{12}\right),
$$

where

$$
f_{e x}\left(\xi, S_{12}\right)=\frac{\xi e^{-\xi} S_{12}}{1-e^{-\xi} S_{12}} \quad \text { and } \quad \xi=\frac{|\vec{b}|^{2}}{a_{R}}=\frac{\left(\vec{r}-a_{I} \vec{p}\right)^{2}}{a_{R}}+a_{R} \vec{p}^{2}
$$

The first two terms in (89) are the same as for distinguishable particles. The remaining part is the exchange term which contains the quantity $\xi$ and the spin overlap $S_{12}=\left|\left\langle\chi_{1} \mid \chi_{2}\right\rangle\right|^{2}$. From Eq. (90) it is evident that $\xi$ may be regarded as a dimensionless measure for the distance in phase space where $\sqrt{a_{R}}$ is the length scale. For large $\xi \gtrsim 4$ the exchange vanishes and the Pauli principle is not active. For small $\xi$ the distance between the fermions is always larger than $|\vec{r}|$ because the exchange term is positive definite. This effect is sometimes called "Pauli repulsion", but this is not a force between the particles.

The analogue expression for the distance in momentum space is

$$
\langle\kappa\rangle=\left(\frac{\left\langle q\left|\vec{k}^{2}\right| q\right\rangle}{\langle q \mid q\rangle}-\frac{3}{2 a_{R}}\right)^{\frac{1}{2}}
$$


with the expectation value for the square momentum

$$
\frac{\left\langle q\left|\underset{\sim}{\vec{k}^{2}}\right| q\right\rangle}{\langle q \mid q\rangle}=\vec{p}^{2}+\frac{3}{2 a_{R}}+\frac{1}{a_{R}} f_{e x}\left(\xi, S_{12}\right) .
$$

Again a term proportional to $f_{e x}\left(\xi, S_{12}\right)$ appears so that there is complete analogy between coordinate and momentum space. The exchange term is again positive and causes a "Pauli repulsion" in relative momentum.

It is interesting to note that for a mininum uncertainty state $|q\rangle$ with $a_{I}=0$ and equal spins $\left(S_{12}=1\right)$ the sum of the observable distances in coordinate and momentum space fulfill

$$
\langle d\rangle^{2} / a_{R}+\langle\kappa\rangle^{2} a_{R}=\xi \frac{1+e^{-\xi}}{1-e^{-\xi}} \geq 2 \text { for } a_{I}=0 .
$$

Different from the measure $\xi$ the observable distance in phase space can never get smaller than 2, for details see subsection Sec. III A 4.

Expression (92) tells also that the kinetic energy of relative motion

$$
\mathcal{T}=\frac{1}{2 \mu} \frac{\left\langle q\left|\vec{\sim}^{2}\right| q\right\rangle}{\langle q \mid q\rangle}=\frac{\vec{p}^{2}}{2 \mu}+\frac{3}{4 \mu a_{R}}+\frac{1}{2 \mu a_{R}} f_{e x}\left(\xi, S_{12}\right)
$$

consists of the classical part $\vec{p}^{2} /(2 \mu)$, the contribution from the uncertainty $3 /\left(4 \mu a_{R}\right)$ and an additional potential $f_{e x}\left(\xi, S_{12}\right) /\left(2 \mu a_{R}\right)$ which depends on $\vec{r}, \vec{p}, a$ and the spin overlap $S_{12}$.

An analogue expression can be obtained for a spin-independent potential which is smooth

$$
\mathcal{V}=\frac{\langle q|V(\underset{\sim}{\vec{x}})| q\rangle}{\langle q \mid q\rangle} \approx V(\vec{r})+\frac{1}{6} \Delta V(\vec{r}) \frac{3|a|^{2}}{2 a_{R}}+\frac{1}{6} \frac{|a|^{2}}{a_{R}} \Delta V(\vec{r}=0) f_{e x}\left(\xi, S_{12}\right) .
$$

This expression is exact if $V(\underset{\sim}{\vec{x}})=V_{0}+V_{2} \underset{\sim}{\vec{x}^{2}}$, and a good approximation if the Taylor expansion up to second order of $V(\underset{\sim}{\vec{x}})$ around $\vec{x}=\vec{r}$ is adequate within the range of the wave packet. Please note that due to rotational symmetry $V(\underset{\sim}{\vec{x}})$ depends only on $\underset{\sim}{\vec{x}^{2}}$.

Combining Eqs. (94) and (95) yields a Hamilton function which splits into three parts

$$
\begin{aligned}
\mathcal{H} & =\mathcal{T}+\mathcal{V}=\mathcal{H}_{\text {classical }}+\mathcal{V}_{\text {uncertainty }}+\mathcal{V}_{\text {Pauli }} \\
& =\left[\frac{\vec{p}^{2}}{2 \mu}+V(\vec{r})\right]+\left[\frac{3}{4 \mu a_{R}}+\frac{|a|^{2}}{4 a_{R}} \Delta V(\vec{r})\right]+\left[\frac{1}{2 \mu a_{R}}+\frac{|a|^{2}}{6 a_{R}} \Delta V(\vec{r}=0)\right] f_{\text {ex }}\left(\xi, S_{12}\right)
\end{aligned}
$$


Eq. (96) is a basis for the concept used by several authors in nuclear (Wilets et al., 1977; Wilets et al., 1978; Boal and Glosli, 1988; Dorso et al., 1987; Dorso and Randrup, 1987; Peilert et al., 1991; Maruyama et al., 1992; Niita et al., 1995) and atomic physics (Klakow et al., 1994; Klakow et al., 1994; Ebeling and Militzer, 1997) to incorporate the uncertainty principle and the Pauli principle in order to extend classical molecular dynamics. A twobody potential $\sum_{i<j} \mathcal{V}_{\text {uncertainty }}\left(\vec{r}_{i j}, \vec{p}_{i j}, a\right)$ is added to simulate the effects of the Heisenberg uncertainty principle and $\sum_{i<j} \mathcal{V}_{\text {Pauli }}\left(\vec{r}_{i j}, \vec{p}_{i j}, a, S_{i j}\right)$ is added to imitate the effects of the Pauli principle. The explicit form needs not to be the one given in (96), but it is usually adapted to the specific use. The method is quite successful in calculating energies (Dorso et al., 1987; Dorso and Randrup, 1987), but we want to advise caution in using the Hamilton function (96) naively in equations of motion like

$$
\dot{\vec{r}}_{i}=\frac{\partial}{\partial \vec{p}_{i}} \mathcal{H} \quad \text { and } \quad \dot{\vec{p}}_{i}=-\frac{\partial}{\partial \vec{r}_{i}} \mathcal{H}
$$

The reason is that $\vec{r}_{i}$ and $\vec{p}_{i}$ are no longer canonical variables. As discussed earlier, although they still define the trial state $|Q\rangle$ uniquely, they loose their intuitive meaning when the particles are indistinguishable. The operator $\underset{\sim}{\vec{x}}(i)$, "position of particle $i$ ", is meaningless and $\langle q|\underset{\sim}{\vec{x}}(i)| q\rangle /\langle q \mid q\rangle$ is not $\vec{r}_{i}$ ! The analogue holds for the momentum. Instead of postulating Eq. (97) we have to go back to the Lagrangian (14) and derive the equations of motion (72). It is evident that the matrix $\mathcal{C}$ will also be changed by the antisymmetrization.

\section{Center of mass motion}

Before discussing the relative motion in the antisymmetric case we consider first the center of mass motion. The Lagrange function (14) for the center of mass wave packet (78) is given by

$$
\mathcal{L}_{c m}=\mathcal{L}_{0 \mathrm{~cm}}-\mathcal{T}_{c m}
$$

with 


$$
\begin{aligned}
\mathcal{L}_{0 c m} & =\frac{i}{2} \frac{\left\langle q_{c m} \mid \dot{q}_{c m}\right\rangle-\left\langle\dot{q}_{c m} \mid q_{c m}\right\rangle}{\left\langle q_{c m} \mid q_{c m}\right\rangle} \\
& =\frac{i}{2} \frac{\left(\vec{B}^{*}-\vec{B}\right)\left(\dot{\vec{B}}^{*}+\dot{\vec{B}}\right)}{A^{*}+A}-\frac{i}{4}\left[\left(\frac{\vec{B}^{*}-\vec{B}}{A^{*}+A}\right)^{2}-\frac{3}{A^{*}+A}\right]\left(\dot{A}^{*}-\dot{A}\right)+\frac{3 i}{4}\left(\frac{\dot{A}}{A}-\frac{\dot{A}^{*}}{A^{*}}\right) \\
& =\vec{P} \cdot \dot{\vec{R}}+\frac{3}{4} \frac{\dot{A}_{I}}{A_{R}}+\text { total time derivative }
\end{aligned}
$$

and the kinetic energy

$$
\mathcal{T}_{c m}=\frac{\left\langle q_{c m}\left|\frac{1}{2 M} \vec{K}_{\sim}^{2}\right| q_{c m}\right\rangle}{\left\langle q_{c m} \mid q_{c m}\right\rangle}=\frac{1}{2 M} \frac{\left(\vec{B}^{*}-\vec{B}\right)^{2}}{A^{*}+A}+\frac{3}{2 M\left(A^{*}+A\right)}=\frac{\vec{P}^{2}}{2 M}+\frac{3}{4 M A_{R}} .
$$

The Euler Lagrange equations yield

$$
\dot{\vec{B}}=0, \quad \dot{A}=\frac{i}{M}
$$

or if one transforms $\vec{B}$ and $\vec{B}^{*}$ into $\vec{R}$ and $\vec{P}$

$$
\dot{\vec{P}}=0, \quad \dot{\vec{R}}=\frac{\vec{P}}{M}, \quad \dot{A}=\frac{i}{M} .
$$

In the center of mass wave function $\vec{R}(t)$ and $\vec{P}(t)$ have always the classical meaning of the mean center of mass position and momentum, respectively. Nevertheless, when the width parameter $A$ is included as a dynamical variable, the wave packet $\left|q_{c m}(t)\right\rangle=|A(t), \vec{B}(t)\rangle$ is the exact solution of the Schrödinger equation.

\section{Relative motion}

The Lagrange function for the relative motion

$$
\begin{aligned}
\mathcal{L}\left(q, q^{*}, \dot{q}, \dot{q}^{*}\right) & =\frac{i}{2}\left(\frac{\langle q \mid \dot{q}\rangle-\langle\dot{q} \mid q\rangle}{\langle q \mid q\rangle}\right)-\frac{\langle q|\underset{\sim}{\stackrel{\vec{k}}{\sim}} / 2 \mu| q\rangle}{\langle q \mid q\rangle}-\frac{\langle q|V(\underset{\sim}{\vec{x}})| q\rangle}{\langle q \mid q\rangle} \\
& \equiv \mathcal{L}_{0}-\mathcal{T}-\mathcal{V}
\end{aligned}
$$

features the antisymmetrization not only in the kinetic and potential energy as seen in Eqs. (94) and (95) but also in the metric part $\mathcal{L}_{0}$.

For the sake of simplicity we treat in this subsection only equal, time-independent spins. In the general case the potential $V(\underset{\sim}{\vec{x}}, \underset{\sim}{\vec{s}}(1), \underset{\sim}{\vec{\sim}}(2))$ may of course depend on spin and the spin 
degrees of freedom. The parameters $\left\langle\uparrow, \downarrow \mid \chi_{1,2}(t)\right\rangle$ would then also appear in $\mathcal{L}_{0}, \mathcal{T}$ and $\mathcal{V}$ and one would get equations of motion for them. For time-independent equal spins $\mathcal{L}_{0}$ is given by

$$
\begin{aligned}
\mathcal{L}_{0}= & \frac{i}{2} \frac{\left(\vec{b}^{*}-\vec{b}\right)\left(\dot{\vec{b}}^{*}+\dot{\vec{b}}\right)}{a^{*}+a}-\frac{i}{4}\left[\left(\frac{\vec{b}^{*}-\vec{b}}{a^{*}+a}\right)^{2}-\frac{3}{a^{*}+a}\right]\left(\dot{a}^{*}-\dot{a}\right) \\
& +\frac{i}{2}\left[\frac{\dot{\vec{b}} \cdot \vec{b}^{*}-\vec{b} \cdot \dot{\vec{b}}^{*}}{\vec{b}^{*} \cdot \vec{b}}+\frac{\dot{a}^{*}-\dot{a}}{a^{*}+a}\right] f_{e x}\left(\xi, S_{12}\right)+\text { total time derivative } \\
= & \vec{p} \cdot \dot{\vec{r}}+\frac{3}{4} \frac{\dot{a}_{I}}{a_{R}}+\left[\frac{\vec{p} \cdot \dot{\vec{r}}-\vec{r} \cdot \dot{\vec{p}}}{\xi}+\frac{\vec{p}^{2}\left(\dot{a}_{R} a_{I}-\dot{a}_{I} a_{R}-\vec{r} \cdot \vec{p} \dot{a}_{R}\right)}{a_{R}}+\frac{\dot{a}_{I}}{a_{R}}\right] f_{e x}\left(\xi, S_{12}\right) \\
& + \text { total time derivative. }
\end{aligned}
$$

As expected, $\mathcal{L}_{0}$ contains an exchange term, besides the terms for distinguishable particles, compare with $\mathcal{L}_{0 \mathrm{~cm}}$ in Eq. (99). Although $\mathcal{L}_{0}$ looks very complicated the Euler Lagrange equations (72)

$$
i \mathcal{C}\left(\begin{array}{c}
\dot{a} \\
\dot{\vec{b}}
\end{array}\right)=\left(\begin{array}{c}
\frac{\partial \mathcal{T}}{\partial a^{*}} \\
\frac{\partial \mathcal{T}}{\partial \vec{b}^{*}}
\end{array}\right)+\left(\begin{array}{c}
\frac{\partial \mathcal{V}}{\partial a^{*}} \\
\frac{\partial \mathcal{V}}{\partial \vec{b}^{*}}
\end{array}\right) \text {. }
$$

can be partially simplified because the free motion is, like for the c.m. state or the case of distinguishable particles, again the exact solution of the Schrödinger equation

$$
\mathcal{C}^{-1}\left(\begin{array}{c}
\frac{\partial \mathcal{T}}{\partial a^{*}} \\
\frac{\partial \mathcal{T}}{\partial \vec{b}^{*}}
\end{array}\right)=\left(\begin{array}{c}
-\frac{1}{\mu} \\
0
\end{array}\right)
$$

and herewith

$$
\left(\begin{array}{c}
\dot{a} \\
\dot{\vec{b}}
\end{array}\right)=\left(\begin{array}{c}
\frac{i}{\mu} \\
0
\end{array}\right)-i \mathcal{C}^{-1}\left(\begin{array}{c}
\frac{\partial \mathcal{V}}{\partial a^{*}} \\
\frac{\partial \mathcal{V}}{\partial \vec{b}^{*}}
\end{array}\right) .
$$

The complicated form of $\mathcal{C}^{-1}$ combines with the complicated derivatives $\left(\frac{\partial \mathcal{T}}{\partial a^{*}}, \frac{\partial \mathcal{T}}{\partial \vec{b}^{*}}\right)$ of the kinetic energy such that the simple result (106) is obtained. Although lengthy to calculate it is easy to understand. The reason is that both states, $|a, \vec{b}\rangle$ and $|a,-\vec{b}\rangle$, are exact solutions of the Schrödinger equation, they only differ in the initial conditions. The antisymmetric state $|q\rangle=|a, \vec{b}\rangle-|a,-\vec{b}\rangle$, defined in (79), is therefore also an exact solution. The 
deeper reason is that both, the time derivative and the hamiltonian, commute with the antisymmetrization operator $\underset{\sim}{A}$ in Eq. (70)

$$
0=i \frac{d}{d t} \underset{\sim}{A}|\Psi(t)\rangle-\underset{\sim}{H} \underset{\sim}{A}|\Psi(t)\rangle=\underset{\sim}{A}\left(i \frac{d}{d t}|\Psi(t)\rangle-\underset{\sim}{H}|\Psi(t)\rangle\right) .
$$

Therefore, if $|\Psi(t)\rangle$ is the exact solution of the Schrödinger equation, so is $\underset{\sim}{A}|\Psi(t)\rangle$. This general statement is not true if $|\Psi(t)\rangle$ is only an approximation.

In the special case of a harmonic interaction, $V(\underset{\sim}{\vec{x}})=V_{0}+V_{2} \underset{\sim}{\vec{x}^{2}}$, the contribution from the potential assumes a very simple form as well, because

$$
\mathcal{C}^{-1}\left(\begin{array}{c}
\frac{\partial\left\langle\vec{x}^{2}\right\rangle}{\partial a^{*}} \\
\frac{\partial\left\langle\vec{x}^{2}\right\rangle}{\partial \vec{b}^{*}}
\end{array}\right)=\left(\begin{array}{c}
2 a^{2} \\
2 a \vec{b}
\end{array}\right) .
$$

The amazing result is that Eqs. (106) and (109) are the same for distinguishable particles and for indistinguishable particles, where $\mathcal{C}^{-1}$ is a complicated matrix depending on $a^{*}, a$ and $\vec{b}^{*}, \vec{b}$. The exchange term in the expression for $\left\langle\underset{\sim}{\vec{k}^{2}}\right\rangle$, Eq. (92), and for $\left\langle\vec{\sim}^{2}\right\rangle$, Eq. (89), compensates for the different $\mathcal{C}$. It is also interesting to see that for a harmonic interaction the parameters $\vec{r}$ and $\vec{p}$ obey the classical equations of motion

$$
\dot{\vec{r}}=\frac{\vec{p}}{\mu}, \quad \dot{\vec{p}}=-2 V_{2} \vec{r}
$$

although the trial state $|q\rangle$ is antisymmetric and describes two identical fermions. This result is, however, only obtained if the width $a$ is at the same time a dynamical variable with the equation of motion

$$
\dot{a}=\frac{i}{\mu}-i 2 V_{2} a^{2}
$$

The case, where $a(t)=a_{0}$ is supposed to be a positive time-independent number, leads to completely different results and is discussed in the following subsection.

\section{Relative motion with a time-independent width parameter}

This section investigates how the equations of motion change if the shape of the relative wave packet, Eq. (79), is restricted further by removing the width degree of freedom $a(t)=$ 
$a_{R}(t)+i a_{I}(t)$ as a dynamical variable. For simplification only parallel spins are considered, i.e. $S_{12}=1$. By setting $a_{R}(t)=a_{0}, a_{I}(t)=0$ and $\dot{a}(t)=0$ in Eqs. (94) and (104) we obtain

$$
\begin{aligned}
\mathcal{L}_{0} & =\vec{p} \cdot \dot{\vec{r}}+(\vec{p} \cdot \dot{\vec{r}}-\vec{r} \cdot \dot{\vec{p}}) \frac{e^{-\xi}}{1-e^{-\xi}} \\
\mathcal{T} & =\frac{\vec{p}^{2}}{2 \mu}+\frac{3}{4 \mu a_{0}}+\frac{1}{2 \mu a_{0}} \frac{\xi e^{-\xi}}{1-e^{-\xi}},
\end{aligned}
$$

where

$$
\xi=\vec{r}^{2} / a_{0}+\vec{p}^{2} a_{0} \text { and } \mathcal{L}=\mathcal{L}_{0}-\mathcal{T}-\mathcal{V}
$$

The potential energy $\mathcal{V}$ is not given here explicitly and the spin dynamics is also not considered for simplicity. The equations of motion for $\vec{r}$ and $\vec{p}$ are

$$
\begin{aligned}
0 & =\frac{d}{d t} \frac{\partial \mathcal{L}}{\partial \dot{\vec{p}}}-\frac{\partial \mathcal{L}}{\partial \vec{p}} \text { or } \\
-\dot{\vec{r}} & -\frac{2 e^{-\xi}}{1-e^{-\xi}}\left(\dot{\vec{r}}-\frac{\vec{r}(\vec{r} \dot{\vec{r}}) / a_{0}+\vec{p}(\vec{p} \dot{\vec{r}}) a_{0}}{1-e^{-\xi}}\right)+\frac{2 e^{-\xi} a_{0}}{\left(1-e^{-\xi}\right)^{2}}(\vec{r}(\vec{p} \dot{\vec{p}})-\vec{p}(\vec{r} \dot{\vec{p}})) \\
& =-\frac{\partial}{\partial \vec{p}} \mathcal{H}(\vec{r}, \vec{p})
\end{aligned}
$$

and

$$
\begin{aligned}
0 & =\frac{d}{d t} \frac{\partial \mathcal{L}}{\partial \dot{\vec{r}}}-\frac{\partial \mathcal{L}}{\partial \vec{r}} \text { or } \\
\dot{\vec{p}} & +\frac{2 e^{-\xi}}{1-e^{-\xi}}\left(\dot{\vec{p}}-\frac{\vec{r}(\vec{r} \dot{\vec{p}}) / a_{0}+\vec{p}(\vec{p} \dot{\vec{p}}) a_{0}}{1-e^{-\xi}}\right)+\frac{2 e^{-\xi}}{\left(1-e^{-\xi}\right)^{2} a_{0}}(\vec{r}(\vec{p} \dot{\vec{r}})-\vec{p}(\vec{r} \dot{\vec{r}})) \\
& =-\frac{\partial}{\partial \vec{r}} \mathcal{H}(\vec{r}, \vec{p}) .
\end{aligned}
$$

For the reduced set of variables we are able to solve Eqs. (115) and (116) for $\dot{\vec{r}}$ and $\dot{\vec{p}}$. The result is

$$
\dot{\vec{r}}=\alpha_{1}(\xi) \frac{\partial \mathcal{H}}{\partial \vec{p}}+\alpha_{2}(\xi)\left\{a_{0}\left(\vec{p} \frac{\partial \mathcal{H}}{\partial \vec{p}}+\vec{r} \frac{\partial \mathcal{H}}{\partial \vec{r}}\right) \vec{p}+\left(\frac{\vec{r}}{a_{0}} \frac{\partial \mathcal{H}}{\partial \vec{p}}-a_{0} \vec{p} \frac{\partial \mathcal{H}}{\partial \vec{r}}\right) \vec{r}\right\}
$$

and

$$
\dot{\vec{p}}=-\alpha_{1}(\xi) \frac{\partial \mathcal{H}}{\partial \vec{r}}+\alpha_{2}(\xi)\left\{\left(\frac{\vec{r}}{a_{0}} \frac{\partial \mathcal{H}}{\partial \vec{p}}-a_{0} \vec{p} \frac{\partial \mathcal{H}}{\partial \vec{r}}\right) \vec{p}-\left(\vec{r} \frac{\partial \mathcal{H}}{\partial \vec{r}}+\vec{p} \frac{\partial \mathcal{H}}{\partial \vec{p}}\right) \frac{\vec{r}}{a_{0}}\right\}
$$


where $\alpha_{1}(\xi)$ and $\alpha_{2}(\xi)$ are functions of $\xi=\vec{r}^{2} / a_{0}+\vec{p}^{2} a_{0}$ and given by

$$
\begin{gathered}
\alpha_{1}(\xi)=\frac{1-e^{-\xi}}{1+e^{-\xi}} \\
\alpha_{2}(\xi)=\frac{2 e^{-\xi}\left(1-e^{-\xi}\right)}{\left(1+e^{-\xi}\right)^{2}\left(1-e^{-\xi}\right)-2 \xi e^{-\xi}\left(1+e^{-\xi}\right)} .
\end{gathered}
$$

Since $\alpha_{1}(\xi \gg 1)=1$ and $\alpha_{2}(\xi \gg 1)=0$ one recognizes for $\xi \gg 1$ Hamilton's equations of motion. $\xi \gg 1$ means that the two fermions are far from each other in phase space. In that limit the identical fermions behave like classical distinguishable particles although their wave function is of course still antisymmetrized. When they get close in phase space, i.e. $\xi<1, \alpha_{1}(\xi) \rightarrow \xi / 2$ and $\alpha_{2}(\xi) \rightarrow 3 / \xi^{2}$ which means that the Hamilton-like parts in (117) and (118) vanish like $\xi / 2$ but the remaining parts increase like $3 / \xi^{2}$.

In this example one sees that for $\xi \lesssim 2$ the equations of motion, which result from the parameterization (79) with $a(t) \equiv a_{0}$, cannot be cast into Hamilton's form when $\vec{r}$ and $\vec{p}$ are regarded as canonical variables. To prove this statement let us suppose that a Hamilton function $\mathcal{H}_{\text {Pauli }}(\vec{r}, \vec{p})$ exists such that Eqs. (117) and (118) can be written as

$$
\dot{r}_{i}=\frac{\partial \mathcal{H}_{\text {Pauli }}}{\partial p_{i}} \text { and } \dot{p}_{i}=-\frac{\partial \mathcal{H}_{\text {Pauli }}}{\partial r_{i}}, i=1,2,3,
$$

where $i$ denotes the three spatial directions. Let us now disprove the existence of a function $\mathcal{H}_{\text {Pauli }}$ by calculating the mixed derivatives $\frac{\partial \dot{r}_{i}}{\partial r_{k}}$ and $\frac{\partial \dot{p}_{k}}{\partial p_{i}}$ which should add to zero if Eq. (121) is true. From the equations of motion (117) and (118) it is easy to verify that

$$
\frac{\partial \dot{r}_{i}}{\partial r_{k}}+\frac{\partial \dot{p}_{k}}{\partial p_{i}} \neq 0
$$

This disproves the existence of a hamiltonian $\mathcal{H}_{\text {Pauli }}(\vec{r}, \vec{p})$ as a function of $\vec{r}$ and $\vec{p}$ which would describe the fermionic dynamics derived from the ansatz (79) with $a(t) \equiv a_{0}$ for the wave function.

It is, however, possible to find a pair of canonical variables $(\vec{\rho}, \vec{\pi})$

$$
\vec{\rho}=\sqrt{\frac{1+e^{-\xi}}{1-e^{-\xi}}} \vec{r} \text { and } \vec{\pi}=\sqrt{\frac{1+e^{-\xi}}{1-e^{-\xi}}} \vec{p}
$$


which are non-linear functions of the original variables $(\vec{r}, \vec{p})$ (Saraceno et al., 1983) such that $\mathcal{L}_{0}$ in Eq. (112) assumes the canonical form

$$
\mathcal{L}_{0}=\frac{1}{2}(\vec{\pi} \cdot \dot{\vec{\rho}}-\vec{\rho} \cdot \dot{\vec{\pi}})+\frac{1}{2} \frac{d}{d t}(\vec{r} \vec{p})
$$

With these new variables the equations of motion aquire the form $(121)$ where $\mathcal{H}_{\text {Pauli }}(\vec{\rho}, \vec{\pi})=$ $\mathcal{H}(\vec{r}, \vec{p})$ is the total energy expressed in the new canonical variables. However, $\mathcal{H}_{\text {Pauli }}(\vec{\rho}, \vec{\pi})$ cannot be expressed in a closed form because Eqs. (123) cannot be solved for $\vec{r}(\vec{\rho}, \vec{\pi})$ and $\vec{p}(\vec{\rho}, \vec{\pi})$.

Equations (121) represent an approach often used in literature to incorporate the effects of the Pauli principle by means of a two-body interaction. Different forms of momentumdependent potentials have been added to the classical hamiltonian or to the lagrangian (Neumann and Fai, 1994). Here we see that this method differs in several aspects from fermionic dynamics. Firstly, as discussed above the dynamical behaviour of $\vec{r}$ and $\vec{p}$ needs not be of Hamilton's type. Secondly, one should not replace $\langle V(\underset{\sim}{\vec{x}})\rangle$ simply by $V(\vec{r})$ because even for narrow wave packets there is an exchange term which is not small for $\xi<1$, see Eq. (96).

The equations of motion simplify appreciably for a time-independent width parameter $a_{0}$, but the price to be paid is that free motion without interaction is not exact anymore. For $\xi<1$ the equations of motion differ essentially from the expected result $\dot{\vec{r}}=\vec{p} / \mu$ and $\dot{\vec{p}}=0$ (see (117) and (118)). The shape of the wave packet is restrained too much so that the particles scatter even if there is no interaction.

There is however the appealing feature of the canonical variables $(\vec{\rho}, \vec{\pi})$ to exhibit a geometrically forbidden region in phase space. From (123) it is evident that $\vec{\rho}^{2} / a_{0}+\vec{\pi}^{2} a_{0} \geq 2$. This is demonstrated in Fig. 4, where in a one-dimensional example several trajectories of the relative motion are shown for two freely moving fermions. The l.h.s. displays the trajectories, which are actually contours of constant kinetic energy, using $r$ and $p$ as dynamical variables, the r.h.s. shows the same trajectories but using $\rho$ and $\pi$. The empty area in the middle of the right-hand figure is the Pauli forbidden region of phase space. It corresponds to the 

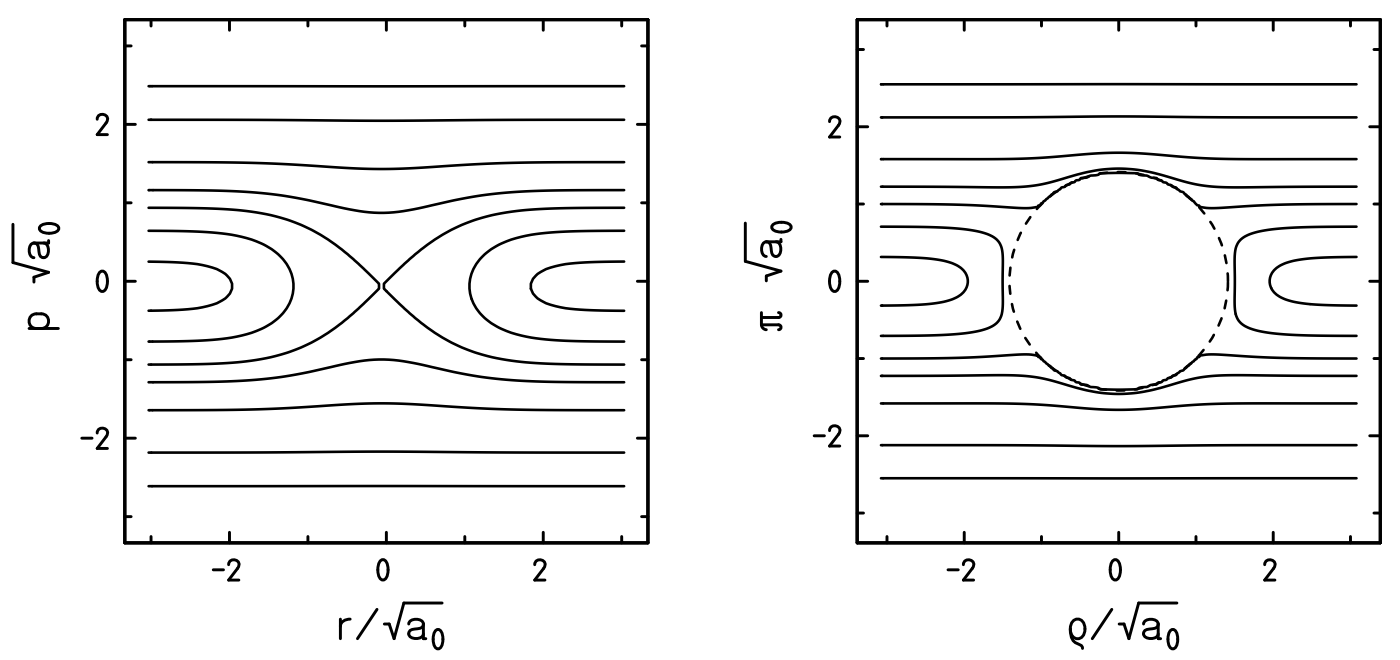

FIG. 4. Trajectories in phase space of one-dimensional free motion with fixed width.

L.h.s.: variables $r$ and $p$. R.h.s.: canonical variables $\rho$ and $\pi$.

single point at the origin $(r=0$ and $p=0)$ in the left-hand figure. The trajectories which cross there, go around the circle in the canonical variables $\rho$ and $\pi$.

Pauli potentials are usually chosen such that a pair of particles acquires a high energy in the forbidden region. One should however be aware that the kinetic energy at the boundary is finite, namely $5 /\left(4 \mu a_{0}\right)$ in the three-dimensional case, see (113). The deficiency, that particles scatter even if there is no interaction, is also present in all Pauli potentials. Inclusion of the complex width $a(t)$ as a dynamical variable cures this problem as has been demonstrated.

At the end of this subsection the reader should not be left with the impression that the Pauli principle is a two-body effect. In fact antisymmetrization is a genuine $N$-body correlation as will be discussed in the following.

\section{Resumé}

1. The intuitive idea to include the Paul principle in a classical description by treating the exchange terms of the relative kinetic and potential energy of two identical fermions 
as an additional "Pauli potential" which supplements the classical equations of motion cannot be supported. It is not correct to regard the parameters $\vec{r}$ and $\vec{p}$ as canonical variables if

$$
\vec{b} \cdot \vec{b}^{*}=|\vec{r}+i a \vec{p}|^{2} \lesssim 2\left|a^{*}+a\right|
$$

and hence it is questionable whether the expectation value of the hamiltonian can be used as the Hamilton function in Hamilton's equations of motion.

2. Heisenberg's quantum uncertainty refers to the variances $\left(\left\langle\underset{\sim}{\vec{x}}{ }^{2}\right\rangle-\langle\underset{\sim}{\vec{x}}\rangle^{2}\right)$ and $\left(\left\langle\underset{\sim}{\vec{k}^{2}}\right\rangle-\right.$ $\langle\underset{\sim}{\vec{k}}\rangle^{2}$ ) of the wave packet which are given by the width parameter $a$ and are not related to $\vec{r}^{2}$ or $\vec{p}^{2}$. It is therefore open to doubt if inclusion of uncertainty into classical equations of motion can be achieved by a potential which depends on $\left(\vec{r}_{i j}^{2} \cdot \vec{p}_{i j}^{2}\right)$. 


\section{B. Effects of antisymmetrization in many-body space}

\section{Shell structure due to antisymmetrization}

It is not immediately obvious that an antisymmetrized product state like Eq. (70) includes shell-model features, like the nodal structure of single-particle orbits, because the states are localized in coordinate and momentum space. But due to the invariance of a Slater determinant under linearly independent transformations among the occupied single-particle states, after antisymmetrization, any set of single-particle states which is complete in the occupied phase space is as good as any other. This applies also to nonorthogonal states. To illustrate this we take four one-dimensional real gaussians with the same real width parameter $a_{0}$ and zero mean momentum and displace them by $d=0.75 \sqrt{a_{0}}$ (see 1.h.s. of Fig. 5). The one-body density can be written in terms of orthonormal states $\left|\psi_{m}\right\rangle$ as

$$
\underset{\sim}{\rho}{ }^{(1)}=\sum_{k, l=1}^{N}\left|q_{k}\right\rangle \mathcal{O}_{k l}\left\langle q_{l}\left|=\sum_{m=1}^{N}\right| \psi_{m}\right\rangle\left\langle\psi_{m}\right|,
$$

where the orthonormal eigenstates of $\underset{\sim}{\rho^{(1)}}$, called natural orbits, are given by the following superposition of gaussians

$$
\left|\psi_{m}\right\rangle=\sum_{k=1}^{N}\left|q_{k}\right\rangle\left(\mathcal{O}^{\frac{1}{2}}\right)_{k m}
$$

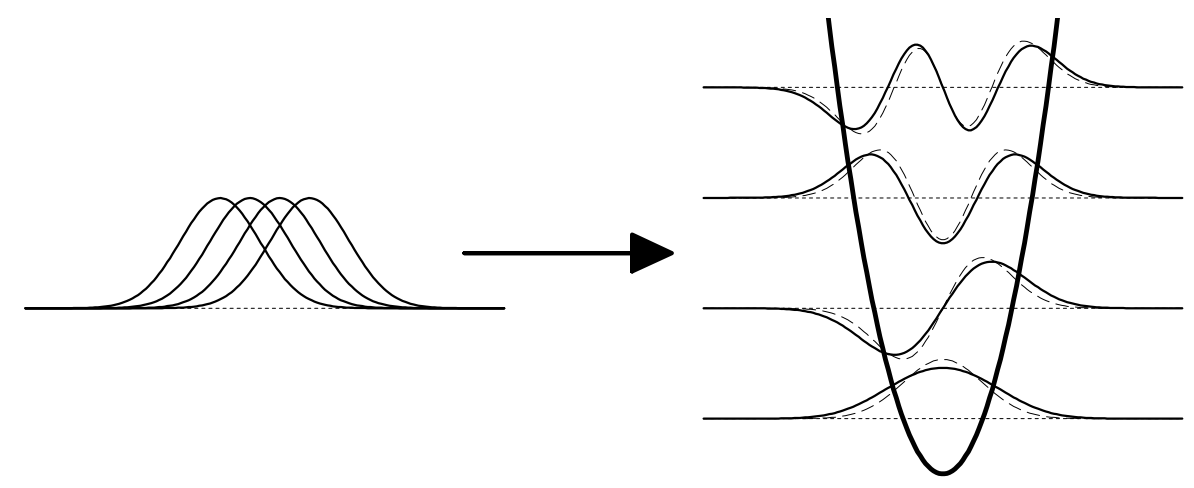

FIG. 5. Natural orbits. Antisymmetrization of four displaced gaussians (1.h.s.) leads to four occupied natural orbits (r.h.s.) which are almost harmonic oscillator states (dashed lines). 
$\mathcal{O}_{k l}$ is the inverse of the overlap matrix, $\left(\mathcal{O}^{-1}\right)_{k l}=\left\langle q_{k} \mid q_{l}\right\rangle$. They are displayed on the right hand side of Fig. 5 and compared to harmonic oscillator eigenstates (dashed lines). One observes that the occupied single-particle states $\left|\psi_{m}\right\rangle$ consist of an s-, p- ,d- and an f-state, all very close to harmonic oscillator states. The difference between both sets can be made arbitrarily small by letting $d / \sqrt{a_{0}}$ approach zero. The f-state is for example essentially the first gaussian minus the second plus the third minus the fourth. All others are similar combinations. As already stressed in the two-body case in the previous section, when the wave packets overlap, $r_{l}$ and $p_{l}$ loose their classical meaning of position and momentum of particle $l$. In the limiting case $d \rightarrow 0$ all $r_{l} \rightarrow 0$ and all $p_{l} \rightarrow 0$ and the harmonic oscillator shells emerge. The distributions in coordinate and momentum space are the quantum mechanically correct ones of four spin polarized fermions in a harmonic oscillator.

\section{Fermi-Dirac distribution due to antisymmetrization}

A second example is illustrated in Fig. 6, where we consider 100 equally spaced gaussians in one dimension. Again all mean momenta are zero and the width $a_{0}$ is real. In the upper part of Fig. 6 the width $\sqrt{a_{0}}$ is 0.2 of the mean distance $d$ so that the wave packets are well separated. Therefore the spatial density $\rho_{x}=\left\langle x\left|\underset{\sim}{\rho^{(1)}}\right| x\right\rangle$ and the momentum density $\rho_{k}=\left\langle k\left|{\underset{\sim}{\rho}}^{(1)}\right| k\right\rangle$ are not changed by antisymmetrization. In the lower part the width has been increased to $\sqrt{a_{0}}=d$. Without antisymmetrization (dash dotted line) the spatial density is uniform and the momentum distribution is that of a single packet. After antisymmetrization (full lines) one obtains the typical shell model oscillations in coordinate space and a Fermi distribution in momentum space. It is amazing to see how in Eq. (126) the superposition of gaussians by means of the inverse overlap matrices can create a fully occupied momentum state, see for example in Fig. 6 the lower right momentum distribution at $k=0.8 k_{F}$, where the individual gaussians give practically zero probability to measure this momentum. We also calculated the eigenstates of the kinetic energy in the occupied space and got perfect sinusoidal waves. 

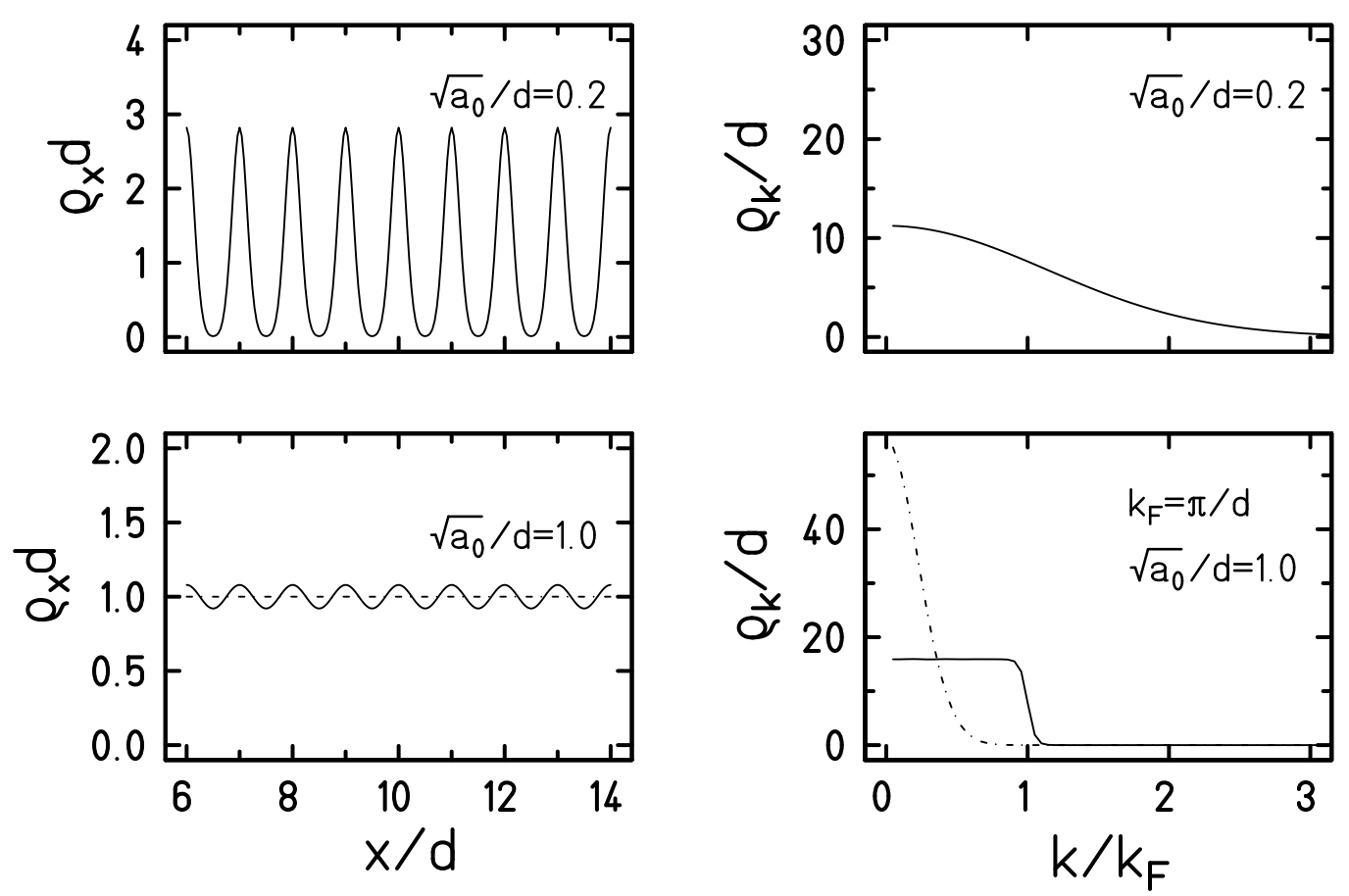

FIG. 6. Densities in coordinate and momentum space. Full line with antisymmetrization, dash-dotted line without. Upper part: section of spatial density of hundred gaussians (not overlapping in coordinate space) and corresponding momentum distribution. Distributions with and without antisymmetrization are identical. Lower part: same as above but for overlapping gaussians. For details see text.

These two examples illustrate nicely that Slater determinants of localized single-particle states with zero mean momentum can describe the harmonic oscillator shell model or even the Fermi motion of a gas of fermions in which plane waves are occupied up to the Fermi momentum. If one wants to simulate this effect by a "Pauli potential", disregarding the momentum distribution in each wave packet, the resulting ground state momentum distribution is unsatisfactory (Dorso et al., 1987). The densities in coordinate and momentum space are just not given by the distributions of the $r_{l}$ and $p_{l}$.

At this point we would like to remind the reader of the different meaning of the three "momentum quantities" used in the article:

1. momentum operator of particle $l$ (not observable): $\underset{\sim}{\vec{k}}(l)=\underset{\sim}{\mathbb{1}} \otimes \cdots \otimes \underset{\sim}{\vec{k}} \otimes \cdots \underset{\sim}{1}$, 
2. momentum variable $\vec{k}$ as used in the momentum representation $\langle\vec{k} \mid q\rangle$ and

3. momentum parameter $\vec{p}_{l}$ which characterizes the state $\left|q_{l}\right\rangle=\left|\vec{r}_{l}, \vec{p}_{l}, a_{l}, \chi_{l}\right\rangle$.

\section{Resumé}

1. The coordinate distribution $\rho_{x}(\vec{x})$ is given by the observable

$$
\rho_{x}(\vec{x})=\frac{\left\langle Q\left|\sum_{l=1}^{N} \delta(\vec{x}-\underset{\sim}{\vec{x}}(l))\right| Q\right\rangle}{\langle Q \mid Q\rangle}
$$

and not by the (eventually time averaged) distribution of the $\vec{r}_{l}$. Analogue the momentum distribution is is given by

$$
\rho_{k}(\vec{k})=\frac{\left\langle Q\left|\sum_{l=1}^{N} \delta(\vec{k}-\underset{\sim}{\vec{k}}(l))\right| Q\right\rangle}{\langle Q \mid Q\rangle}
$$

and not by the distribution of the $\vec{p}_{l}$. All $\vec{p}_{l}$ may be zero and there is still Fermi motion.

2. Fermi motion is not random motion in $\vec{r}_{l}$ and $\vec{p}_{l}$.

3. $\vec{r}_{l}$ and $\vec{p}_{l}$ resume the classical meaning only when the system is so dilute in phase space that the Pauli principle has no consequences any more.

\section{Dynamical considerations}

The equations of motion for the most simple antisymmetric trial state (70) are, see Eq. (72),

$$
i \sum_{\nu} \mathcal{C}_{\mu \nu}\left(Q^{*}, Q\right) \dot{q}_{\nu}=\frac{\partial \mathcal{T}}{\partial q_{\mu}^{*}}+\frac{\partial \mathcal{V}}{\partial q_{\mu}^{*}} .
$$

Like in the two-body case, applying the inverse of $\mathcal{C}\left(Q^{*}, Q\right)$ to the derivatives of the kinetic energy yields the simple result 


$$
\left(\begin{array}{c}
\dot{a}_{1} \\
\dot{\vec{b}}_{1} \\
\dot{\chi}_{1} \\
\dot{a}_{2} \\
\dot{\vec{b}}_{2} \\
\dot{\chi}_{2} \\
\vdots
\end{array}\right)=\left(\begin{array}{c}
\frac{i}{m} \\
0 \\
0 \\
\frac{i}{m} \\
0 \\
0 \\
\vdots
\end{array}\right)-i \mathcal{C}^{-1}\left(\begin{array}{c}
\frac{\partial \mathcal{V}}{\partial a_{1}^{*}} \\
\frac{\partial \mathcal{V}}{\partial \vec{b}_{1}^{*}} \\
\frac{\partial \mathcal{V}}{\partial \chi_{1}^{*}} \\
\frac{\partial \mathcal{V}}{\partial a_{2}^{*}} \\
\frac{\partial \mathcal{V}}{\partial \vec{b}_{2}^{*}} \\
\frac{\partial \mathcal{V}}{\partial \chi_{2}^{*}} \\
\vdots
\end{array}\right) .
$$

Although in the case of free motion $\vec{r}_{l}$ and $\vec{p}_{l}$ follow the classical path, this is once more the exact solution of the Schrödinger equation with $\dot{a}_{l}=i / m$ and $\vec{b}_{l}$ and the spin parameters $\chi_{l}$ being constant. The reason is of course that the many-body time evolution operator, which commutes with the antisymmetrization operator, is a product of single-particle time evolution operators and the free single-particle motion of gaussians is exact.

The Pauli principle appears, like in the two-body case, in the interaction part in a twofold way. The interaction energy $\mathcal{V}\left(Q^{*}, Q\right)$ has exchange terms and the metric $\mathcal{C}^{-1}\left(Q^{*}, Q\right)$ couples all generalized forces $\frac{\partial \mathcal{V}}{\partial q_{\mu}^{*}}$ because it is in general not diagonal in the particle indices. Therefore the second term on the r.h.s. of Eq. (131) acts like a $N$-body force.

Looking at the structure of Eq. (131) it seems more natural to substitute the second term on the r.h.s. by a two-body interaction instead of considering the structure of the kinetic energy as done in Sec. III A. In nuclear physics Wilets and coworkers (Wilets et al., 1977; Wilets et al., 1978) were the first who proposed a space and momentum dependent two-body "Pauli core" which was applied to atomic physics later (Wilets and Cohen, 1998). 


\section{MODELS IN NUCLEAR AND ATOMIC PHYSICS}

In nuclear physics at non-relativistic energies the nucleons cannot be treated as classical particles on trajectories. Their phase space density is too large to ignore the Pauli principle. Therefore various molecular dynamics models which incorporate the Pauli principle on different levels of sophistication are proposed in the literature. They will be discussed in more detail in the following subsections.

Molecular dynamics calculations are also widespread in atomic physics to describe interacting atoms and molecules. But here the degrees of freedom are usually the classical c.m. positions and velocities of the nuclei. The quantal electrons which move in the electric field of the atoms and provide the attraction are often treated by mean-field methods. There are also applications in which each electron is treated as an individual entity localized in phase space. Like for nucleons, if the density is high enough, they can become a degenerate Fermi gas for which a classical molecular dynamics picture cannot be applied.

\section{A. Antisymmetrized wave packets in nuclear physics}

The time-dependent Hartree Fock (TDHF) method (Davies et al., 1985) was expected to be suited for the description of colliding nuclei because stationary Hartree Fock calculations represent successfully ground state properties for all nuclei. The TDHF equations are obtained from the variational principle Eq. (4) when the single-particle states $|q\rangle$ which form the single Slater determinant $|Q\rangle$ are varied in an unrestricted way. However, it turned out that, even at low beam energies where the relative speed between the two colliding nuclei is small compared to the Fermi velocity and hence a mean field is always well established, the amount of fluctuations in the collective variables, like energy loss, scattering angle or mass distribution, was much too small compared to measured data (Davies et al., 1985; Balian and Veneroni, 1992; Reinhard and Suraud, 1992; Lacroix, Chomaz, and Ayik, 1998, 1999). Also the inclusion of collision terms, extended TDHF (see proceedings of "Time-dependend 
Hartree-Fock and beyond" (Goeke and Reinhard, 1982)), did not improve the situation. The reason is that the TDHF equations contain a common mean field which does not allow fluctuations to grow. As discussed in Sec. II C 2 quantum branching into other Slater determinants with different mean fields is missing. Even the collision integral which induces fractional occupation of states and hence a mixing of Slater determinants does not change that because one common mean field is again calculated from this mixture.

Calculations which treat the TDHF time-evolution for each member of a thermal ensemble with its own specific mean field (Knoll and Strack, 1984; Knoll and Wu, 1988) can describe large fluctuations which develop during the expansion. The initial ensemble of Slater determinants, which could be envisaged as the result of quantum branching, was only assumed but not dynamically calculated.

The shortcomings of a common mean field applies also to models which solve the Vlasov equation augmented by a collision integral like VUU (Vlasov Uehling Uhlenbeck) or BUU (Boltzmann Uehling Uhlenbeck), see for instance (Aichelin and Bertsch, 1985; Stöcker and Greiner, 1986; Bertsch and Das Gupta, 1988; Wolf et al., 1990; Gaitanos et al., 1999). Although no explicit antisymmetrization like in TDHF is present, Liouville's theorem and Pauli blocking in the collision term prevent an overoccupation of the single-particle phase space. The inherent lack of fluctations in these one-body descriptions led to molecular dynamics models for fermions which will be discussed in the following subsections.

\section{Time-dependent $\alpha$-cluster model}

The first molecular dynamics model which uses antisymmetrized many-body states of localized constituents in nuclear physics is an extension of the $\alpha$-cluster model, which successfully characterizes $\alpha$-particle nuclei, to the time-dependend case. In the model nuclei are represented as Slater determinants of wave packets for $\alpha$-particles. The width parameter of the gaussian single-particle wave packets is chosen to be fixed (Caurier et al., 1982; Saraceno et al., 1983) as well as time-dependent (Drożdż et al., 1982). Even superpositions 
of two Slater determinants of wave packets for $\alpha$-particles with time-dependent width are investigated (Bauhoff et al., 1985). Since these models are applicable only for nuclei with pronounced $\alpha$-substructure new models were developed in the late eighties, which address one wave packet to each single nucleon.

\section{Fermionic Molecular Dynamics - FMD}

The model of Fermionic Molecular Dynamics (FMD) was suggested in 1990 (Feldmeier, 1990; Feldmeier et al., 1995; Feldmeier and Schnack, 1997) in order to describe ground states of atomic nuclei and heavy-ion reactions in the energy regime below particle production. The many-body trial state of FMD is a Slater determinant $|Q(t)\rangle$ of singleparticle gaussian wave packets $\left|q_{l}(t)\right\rangle$ where $q_{l}(t)$ denotes the set of single-particle param-

eters $q_{l}(t)=\left\{\vec{b}_{l}(t), a_{l}(t), \chi_{l}(t), \xi_{l}\right\}, \vec{b}_{l}(t)=\vec{r}_{l}(t)+i a_{l}(t) \vec{p}_{l}(t)$, which contains mean position, mean momentum and complex width. The spin degrees of freedom are represented by a spinor $\left|\chi_{l}(t)\right\rangle$ (see Eq. (71)). The isospin part $\left|\xi_{l}\right\rangle$ is taken to be time-independent and identifies either a proton or a neutron.

The equations of motion for all parameters are obtained from the variational principle as described in Sec. II (see Eq. (22)). The hamiltonian is an effective one, because the strong short range repulsion in the nucleon-nucleon interaction causes correlations which cannot be described by the trial state $|Q(t)\rangle$ (Feldmeier et al., 1998). Also the spin correlations caused by the strong tensor force are only poorly represented in a single Slater determinant.

In FMD the ground state is defined by the deepest minimum of the energy $\mathcal{H}\left(Q^{*}, Q\right)=$ $\langle Q|\underset{\sim}{H}| Q\rangle$. Since all generalized forces $\partial \mathcal{H} / \partial q_{l}^{*}=0$ vanish in the minimum this state is stationary, all $\dot{q}_{l}=0$. There is no "Fermi motion" in the parameters $\vec{r}_{l}, \vec{p}_{l}, \cdots$. Ground state properties like binding energies and rms radii can be reproduced equally well with a variety of effective nucleon-nucleon interactions which differ mainly in their momentum dependence. But the intrinsic structure of the nuclei depends on the interaction. Superpositions of singleparticle states as well as of Slater determinants can be used in order to obtain a more refined 
description of nuclear structure, e.g. for halo nuclei (Neff et al., 1999).

FMD is able to model a variety of heavy-ion reactions ranging from fusion to dissipative reactions and multifragmentation (Feldmeier and Schnack, 1997). Different from TDHF fluctuations occur in these reactions but the results show also that initial correlations given by the intrinsic structure of the ground states play a major rôle in the simulation of fragmentation reactions (Neff et al., 1999).

The time-dependent width parameters is an important non-classical degree of freedom (Feldmeier et al., 1995), especially to allow for evaporation of nucleons, a process that otherwise is strongly hindered, because each escaping wave packet takes away at least its zero-point energy. Inside a nucleus this zero-point energy is typically $10 \mathrm{MeV}$ but evaporated nucleons have on the mean only $2 \mathrm{MeV}$ kinetic energy. Therefore the packet has to spread during the evaporation process.

Themodynamic equilibrium properties can be determined in FMD by means of time averaging, see Sec. V C and Ref. (Schnack and Feldmeier, 1997).

As already explained in Sec. II C 2 the restricted parameterization leads to barriers which for the exact solution would not exist. Especially the splitting of wave packets is an important source for quantum branching. The lack of this dynamical freedom is a serious hindrance of forming clusters (Kiderlen and Danielewicz, 1996) which is also observed in Antisymmetrized Molecular Dynamics and FMD investigations of spinodal decomposition of nuclear matter and quenches possibly important reaction mechanisms in multifragmentation reactions (Colonna and Chomaz, 1998). Further development in this direction is needed.

\section{Antisymmetrized Molecular Dynamics - AMD}

Antisymmetrized Molecular Dynamics (AMD) is similar to FMD with respect to the choice of the trial state but includes random branching between trial states. For details the reader is referred to Refs. (Ono et al., 1992a; Ono et al., 1992b; Ono et al., 1993; Ono and 
Horiuchi, 1996a; Ono and Horiuchi, 1996b). AMD describes the nuclear many-body system by a Slater determinant $|Q(t)\rangle$ of gaussian wave packets chracterized by the parameter set $q_{l}(t)=\left\{\vec{Z}_{l}(t), \chi_{l}, \xi_{l}\right\}$, where in AMD notation the complex parameters $\vec{Z}_{l}=\frac{1}{\sqrt{2 a_{0}}} \vec{b}_{l}=$ $\frac{1}{\sqrt{2 a_{0}}} \vec{r}_{l}+i \sqrt{\frac{a_{0}}{2}} \vec{p}_{l}$ denote the time-dependent centroids of the wave packets and $\chi_{l}, \xi_{l}$ the timeindependent spin-isospin components which can be either proton or neutron, spin up or down. The width parameter $\nu=\frac{1}{2 a_{0}}$ is real and time-independent and the same for all wave packets.

The time evolution of the $\vec{Z}_{l}(t)$ is determined by the time-dependent variational principle Eq. (4) which leads to the equations of motion given in Eq. (22). The Hamilton function $\mathcal{H}$ used in AMD is the expectation value of a hamiltonian $\underset{\sim}{H}$ plus an additional term which removes the spurious zero-point energy of the center of mass wave packets for the different clusters. This c.m. energy, which is of the order of $10 \mathrm{MeV}$ for all clusters, is an artifact of all product states.

The smooth variation of the $\vec{Z}_{l}(t)$ due to the generalized forces $\partial \mathcal{H} / \partial \vec{Z}_{l}^{*}$ is supplemented by different stochastic forces. These can be regarded as a phenomenological ansatz for quantum branching between different trial states $\left|Q_{j}(t)\right\rangle$ as discussed in Sec. IIC 2. One branching procedure takes care of deviations caused by the short range repulsion between nucleons. For that a collision term is introduced which randomly changes the relative canonical momenta of a pair of wave packets. In order not to enter Pauli-forbidden regions in phase space approximate canonical variables are used which for two particles reduce to those discussed in Sec. III A 4 , compare Fig. 4.

Another branching simulates the spreading and splitting of wave packets, which is an essential process for an adequate description of evaporation and absorption, but cannot be accomplished by the trial state (Ohnishi and Randrup, 1995; Ono and Horiuchi, 1996a; Ohnishi and Randrup, 1997; Ohnishi and Randrup, 1997).

AMD is able to reproduce the essential properties of nuclear ground states (minima in $\left.\mathcal{H}\left(Q^{*}, Q\right)\right)$ and, when extended to trial states which use superpositions of single-particle states or Slater determinants, even the structure of halo nuclei, see e.g. (Kanada-En'yo et 
al., 1995).

Multifragmentation reactions are investigated for beam energies around the Fermi energy. Before comparing with experimental data from heavy-ion collisions the result of a simulated collision is fed into a statistical decay program to account for long time processes (Ono, 1998; Pühlhofer, 1977).

Since the numerical effort of Antisymmetrized Molecular Dynamics as well as of FMD grows with $N^{4}$, for the calculation of systems with more than $N=80$ nucleons approximations are needed. Recently the AMD group developed a "triple loop approximation", which converts the fourfold sum of the potential energy (see Eq. (75)) into a threefold one, so that systems like $\mathrm{Au}+\mathrm{Au}$ are feasible now (Ono, 1998). 


\section{B. Product states of wave packets - QMD}

Models which parameterize the many-fermion trial state by a simple product of gaussian wave packets are called Quantum Molecular Dynamics in nuclear physics. First QMD versions were invented in the eighties (Aichelin and Stöcker, 1986; Aichelin et al., 1987; Aichelin, 1991; Khoa et al., 1992). They all employ a product state

$$
|Q(t)\rangle=\left|q_{1}(t)\right\rangle \otimes\left|q_{2}(t)\right\rangle \otimes \cdots \otimes\left|q_{N}(t)\right\rangle
$$

of single-particle states $\left|q_{l}(t)\right\rangle=\left|\vec{r}_{l}(t), \vec{p}_{l}(t)\right\rangle$ defined in Eq. (45), where only the mean positions $\vec{r}_{l}(t)$ and the mean momenta $\vec{p}_{l}(t)$ are time-dependent. The width is fixed and the same for all wave packets.

The resulting equations of motion are the classical ones, given in Eq. (53). Also the interpretation of $\vec{r}_{l}(t)$ and $\vec{p}_{l}(t)$ is purely classical and the particles are considered distinguishable. This simplifies the collision term, which acts as a random force, and at higher energies the description of transitions from nucleons into resonances.

All QMD versions use a collision term with Pauli blocking in addition to the classical dynamics. Some versions consider spin and isospin, others use nucleons with an average electric charge. Several QMD versions try to incorporate the Pauli principle by means of a Pauli potential that prevents nucleons of the same kind to come too close in phase space, see Sec. III A. Due to these simplifications QMD has the advantage that the numerical effort grows only with $N^{2}$ and thus allows the simulation of large systems. In addition all QMD versions use a statistical decay program for the long time dynamics.

\section{Versions}

QMD models are widely used in nuclear physics and exist in many versions. We would like to mention some of them and apologize for not mentioning all others. A more elaborate overview on QMD models is provided in Ref. (Hartnack et al., 1998). 
1. Using experience with VUU/BUU models (Aichelin and Bertsch, 1985) one of the first QMD versions (Aichelin and Stöcker, 1986; Aichelin, 1991) was suggested. It exploited the trial state (132) only insofar that the interaction gets an effective range due to the folding with the wave packets. In any other respect the model propagates point particles on classical trajectories. The zero-point energy originating from localization is omitted. As initial states random distributions of mean coordinates and momenta are taken according to the experimental ground-state density profile and binding energy. This distribution is however not the ground state of the model hamiltonian but an unstable excited state. The model ground state is highly over-bound. This QMD version does not distinguish between protons and neutrons, all nucleons carry an average charge.

2. The IQMD version treats the isospin explicitly but is the same in all other respects. The version was designed for the analysis of collective flow and pions (Hartnack et al., 1989).

3. Another branch of the QMD evolution uses a Pauli potential and takes the trial state (132) more seriously (Peilert et al., 1992; Konopka et al., 1995). Since nucleons are kept apart in phase space by the Pauli potential (Peilert et al., 1991) the minimum of the hamiltonian determines the nuclear ground state. This is not a mere theoretical beauty, but very important if one wants to investigate the survival of initial groundstate correlations in the final products. An ensemble of random initial states is not able to answer such questions.

4. In the same spirit the Japanese QMD (Maruyama et al., 1992; Niita et al., 1995; Chiba et al., 1996; Maruyama et al., 1996) is constructed. Supplementary the width parameter which is time-independent in all other versions is chosen to be time-dependent here (EQMD). Analogous to AMD the c.m. zero-point energies are subtracted from the hamiltonian, which is difficult because of the changing width. Some observations 
in EQMD are similar to those in FMD. Especially evaporation processes are described much better than with fixed width. Also for fusion reactions the time-dependent width plays a major rôle because the fusion cross sections are too small with fixed width.

5. Another version of QMD was developed in Copenhagen and called NMD (Bondorf et al., 1995).

6. Many attempts have been undertaken to extend the applicability of QMD towards higher (relativistic) energies. Models are for instance RQMD (Sorge et al., 1989; Lehmann et al., 1995; Sorge, 1995) and UrQMD (Bass et al., 1998).

\section{Decoupling of center of mass and relative motion}

A prominent problem of many-body trial states expressed in terms of single-particle quantities, irrespective whether they are antisymmetrized or not, is the center of mass motion which does not separate from the relative motion. One attempt to solve the problem is the construction of a trial state, where the single-particle width parameters are replaced by a width matrix $A_{k l}(t)$ (Kiderlen and Danielewicz, 1996)

$\left\langle\vec{x}_{1}, \ldots, \vec{x}_{N} \mid Q(t)\right\rangle=\exp \left\{-\sum_{k, l}\left(\vec{x}_{k}-\vec{r}_{k}(t)\right) A_{k l}(t)\left(\vec{x}_{l}-\vec{r}_{l}(t)\right)+i \sum_{k} \vec{p}_{k}(t) \vec{x}_{k}\right\}|\chi\rangle$,

$|\chi\rangle$ is a normalized spin-isospin state. The dynamical freedom of the matrix elements $A_{k l}(t)$ allows this state to factorize into c.m. and intrinsic degrees of freedom for subgroups of particles. For two particles the advantage of ansatz (133) can be seen immediately

$$
\left\langle\vec{x}_{1}, \vec{x}_{2} \mid Q\right\rangle=\exp \left\{-A_{c m}(\vec{X}-\vec{R})^{2}+i \vec{P} \vec{X}\right\} \exp \left\{-A_{r e l}(\vec{x}-\vec{r})^{2}+i \vec{p} \vec{x}\right\}|\chi\rangle .
$$

With the usual definitions of relative $(\vec{x}, \vec{r}, \vec{p})$ and c.m. $(\vec{X}, \vec{R}, \vec{P})$ coordinates the width matrix-elements are related by $A_{c m}=2\left(A_{11}+A_{12}\right), A_{\text {rel }}=\left(A_{11}-A_{12}\right) / 2$, and $A_{11}=A_{22}$. Independent of the relative motion, which can be in a bound state with width $A_{\text {rel }}$, the c.m. wave packet width $A_{c m}(t)$ can spread according to free motion. For product states the variance in the c.m. coordinate is always connected to that of the relative motion. For the 
product of two identical gaussian wave packets the relation is $A_{c m}(t)=4 A_{\text {rel }}(t)$ (Kiderlen and Danielewicz, 1996).

The separation of internal and c.m. variables should enhance fragment production which is otherwise suppressed due to the localization energy of the c.m. motion. The authors could demonstrate a significant improvement in the description of light fragments. Unfortunately the proposed ansatz could not be extended to antisymmetrized states up to now. The trial state (133) is also not flexible enough to describe the splitting of wave packets neccessary to model particle capture as discussed in connection with quantum branching in Sec. II C 2 .

\section{Approximate canonical variables - Pauli potential}

The historically first models that tried to describe fragmentation reactions on the basis of single-particle motion were classical models, see for instance (Bodmer and Panos, 1977; Bodmer et al., 1980). Problems arising from the fact, that classical particles neither obey the Heisenberg uncertainty relation nor the Pauli principle of identical fermions are attacked by introducing two-body interactions $\sum_{i<j} \mathcal{V}_{\text {uncertainty }}\left(\vec{r}_{i j}, \vec{p}_{i j}, a\right)$ (Wilets et al., 1977; Wilets et al., 1978) and $\sum_{i<j} \mathcal{V}_{\text {Pauli }}\left(\vec{r}_{i j}, \vec{p}_{i j}, a, S_{i j}\right)$ (Wilets et al., 1977; Wilets et al., 1978; Dorso et al., 1987; Dorso and Randrup, 1987; Boal and Glosli, 1988; Peilert et al., 1991; Maruyama et al., 1992; Niita et al., 1995; Ebeling and Militzer, 1997) which imitate the two quantum effects, see Eq. (96).

The method is quite successful in calculating energies and reasonable single-particle occupations in momentum space for the free Fermi gas at finite temperatures (Dorso et al., 1987; Dorso and Randrup, 1987), but as already mentioned in Sec. III A, care should be taken in using these variables in Hamilton's equation of motion as if they were canonical. In addition classical models cannot correctly describe quantum statistical properties in general, like occupation numbers, mean energy, specific heat etc.

Nevertheless, the idea of simulating Heisenberg uncertainty and Pauli exclusion principle by means of two-body interactions is still being used in nowadays applications of classical 
dynamics to many-fermion problems, e.g. (Latora et al., 1994; Wilets and Cohen, 1998). 


\section{Atomic physics}

In atomic physics molecular dynamics applications are widespread because the de Broglie wave length of the atoms (molecules) is often much shorter than variations in the intermolecular potential. Therefore a trial state for the center of mass coordinates, which is a product of well localized gaussians as discussed in Sec. II B 2, is well suited

$$
\left|Q_{\text {atom }}^{\prime}\right\rangle=\left|\vec{B}_{1}\right\rangle \otimes \cdots \otimes\left|\vec{B}_{N}\right\rangle \text { with } \vec{B}_{l}=\vec{R}_{l}+i A_{0} \vec{P}_{l}
$$

The electrons are much lighter so that quantum effects like Pauli principle and uncertainty are important. Their trial state might be thought of as an antisymmetrized many-body state $\left|Q_{e l}^{\prime} ; Q_{a t o m}^{\prime}\right\rangle$ which depends on electronic degrees of freedom summarized in the set $Q_{e l}^{\prime}=\left\{q_{0}, q_{1}, q_{2}, \cdots\right\}$, e.g. characterizing different orbits. It also depends on the variables of the atoms $\left\{\vec{R}_{l}, \vec{P}_{l}\right\}$ which mark for example the phase space centers of the orbits. The dependence on $\vec{P}_{l}$ is usually neglected because the velocities of the electrons are much larger than those of the atoms. The total trial state is the product

$$
\left|Q^{\prime}\right\rangle=\left|Q_{\text {atom }}^{\prime}\right\rangle \otimes\left|Q_{e l}^{\prime} ; Q_{\text {atom }}^{\prime}\right\rangle
$$

Different from nuclear physics the hamiltonian is known. It can be well approximated by (spin and other relativistic effects neglected)

$$
\begin{aligned}
\underset{\sim}{H} & =\sum_{l=1}^{N}\left[\frac{\vec{\sim}^{2}(l)}{2 M_{l}}-\sum_{i=1}^{N_{e l}} \frac{Z_{l} e^{2}}{|\underset{\sim}{\vec{X}}(l)-\underset{\sim}{\vec{x}}(i)|}\right]+\sum_{l<k}^{N} \frac{Z_{l} Z_{k} e^{2}}{|\underset{\sim}{\vec{X}}(l)-\underset{\sim}{\vec{X}}(k)|}+{\underset{\sim}{H}}_{e l} \\
\underset{\sim}{H_{e l}} & =\sum_{i=1}^{N_{e l}} \underset{\underset{\sim}{\vec{k}^{2}}(i)}{2 m_{e l}}+\sum_{i<j}^{N_{e l}} \frac{e^{2}}{\underset{\sim}{\vec{x}}(i)-\underset{\sim}{\vec{x}}(j) \mid},
\end{aligned}
$$

where capital and small letters denote atomic and electronic variables, respectively. From the Lagrange function $\mathcal{L}^{\prime}$, Eq. (3), one obtains with the appropriate approximations the well known Quantum Molecular Dynamics equations for atomic physics. The atomic variables $\vec{R}_{l}$ and $\vec{P}_{l}$ follow classical equations of motion (width $A_{0}$ small) under the influence of electron potentials given by 


$$
\begin{aligned}
\mathcal{V}_{\text {atom-el }} & =\left\langle Q_{e l}^{\prime} ; Q_{\text {atom }}^{\prime}\left|\sum_{l=1}^{N}\left[-i \underset{\vec{B}_{l}}{\vec{B}_{l}}+\sum_{i=1}^{N_{e l}} \frac{Z_{l} e^{2}}{\left|\vec{R}_{l}-\underset{\sim}{\vec{x}}(i)\right|}\right]\right| Q_{e l}^{\prime} ; Q_{a t o m}^{\prime}\right\rangle \\
& +\left\langle Q_{e l}^{\prime} ; Q_{\text {atom }}^{\prime}\left|\underset{\sim}{H_{e l}}\right| Q_{e l}^{\prime} ; Q_{\text {atom }}^{\prime}\right\rangle .
\end{aligned}
$$

The coupled equations of motion for the electronic degrees of freedom, $Q_{e l}^{\prime}$, are approximated at various levels of sophistication.

These quantum molecular dynamics models are not subject of this review because they do not treat the electrons (fermions) as being localized in phase space.

\section{Product states of wave packets in atomic physics}

For simple Coulomb systems (Suarez Barnes et al., 1993) and for hydrogen plasmas models are proposed which employ trial states of the type

$$
\left|Q^{\prime}\right\rangle=\left|Q_{\text {atom }}^{\prime}\right\rangle \otimes\left|Q_{e l}^{\prime}\right\rangle
$$

where the electronic state $\left|Q_{e l}^{\prime}\right\rangle$ consists of localized gaussian wave packets, Eq. (45). These models are QMD models in the sense of Sec. IV B, either with time-independent width and without Pauli potential (Ebeling and Militzer, 1997) or with Pauli potential (Ebeling and Schautz, 1997) or with time-dependent width and Pauli potential (Klakow et al., 1994; Klakow et al., 1994). Only the electronic part is represented by a wave function, the protons of the hydrogen plasma move on classical trajectories.

An interesting application, which is referred to in Sec. V C 2, is the study of a plasma under extreme conditions, high temperature or pressure and phase transitions to the liquid and solid phase (Klakow et al., 1994; Klakow et al., 1994). Other investigations focus on the degree of ionization of a partially ionised plasma (Ebeling et al., 1996; Ebeling and Militzer, 1997). 


\section{Quantum branching}

When the Born-Oppenheimer approximation is valid adiabatic energy surfaces can be calculated as

$$
\mathcal{V}_{a d}\left(Q_{\text {atom }}^{* *}, Q_{\text {atom }}^{\prime} ; \nu_{e l}\right)=\left\langle\nu_{e l} ; Q_{\text {atom }}^{\prime}\left|\sum_{l=1}^{N} \sum_{i=1}^{N_{e l}} \frac{Z_{l} e^{2}}{\left|\vec{R}_{l}-\underset{\sim}{\vec{x}}(i)\right|}+\underset{\sim}{H_{e l}}\right| \nu_{e l} ; Q_{\text {atom }}^{\prime}\right\rangle,
$$

where $\nu_{e l}=0$ denotes the lowest energy state of the electrons under the influence of the charges $Z_{l}$ of static ions positioned at $\vec{R}_{l} . \nu_{e l}=1,2, \cdots$ numerate the excited eigenstates of the electronic system, for example vibrational modes or particle-hole excitations. In principle one can set up an improved trail state as a linear combination

$$
\left|Q^{\prime}\right\rangle=\sum_{\nu_{e l}} q_{\nu_{e l}}\left|Q_{a t o m, \nu_{e l}}^{\prime}\right\rangle \otimes\left|\nu_{e l} ; Q_{a t o m, \nu_{e l}}^{\prime}\right\rangle
$$

and then try to solve the coupled equations which result from the variational principle (9) for the complex amplitudes $q_{\nu_{e l}}$ and the ion variables $Q_{a t o m, \nu_{e l}}^{\prime}=\left\{\vec{R}_{l}^{\nu_{e l}}, \vec{P}_{l}^{\nu_{e l}} ; l=1, \cdots, N ; \nu_{e l}=\right.$ $1,2, \cdots\}$. From energy, momentum, and angular momentum conservation it is obvious that

one has to have as many different trajectories $\left\{\vec{R}_{l}^{\nu_{e l}}, \vec{P}_{l}^{\nu_{e l}}\right\}$ for the ions as there are excited states which can be populated, because an inelastic excitation $\nu_{e l} \rightarrow \nu_{e l}^{\prime}$ will change the ion trajectories accordingly.

But these equations of motion are usually too complex to be solved numerically, one therefore introduces quantum branching (as discussed in Sec. II C 2) called quasiclassical trajectory surface hopping method. See for example references in (Topaler et al., 1997). Different approaches are tested against an accurate quantum dynamics calculation of a realistic system by Topaler et al. (Topaler et al., 1997). They find fair agreement between the quantum branching methods and results of the quantum equations which result from a trial state of type Eq. (142). This is expected if electronic coherence is not important on time scales of the ion motion so that the hopping between the energy surfaces (141) acts as a random Langevin force on the ion trajectories. 


\section{STATISTICAL PROPERTIES}

Molecular dynamics models are not only used to simulate nonequilibrium but also equilibrium situations, especially when correlations require descriptions which go beyond meanfield approaches and quasi-particles. In the context of classical mechanics a vast literature exists (Hoover, 1985), even for relativistic cases (Belkacem et al., 1998), in which equilibrium properties are studied. So called thermostated time evolutions (Nosé, 1984; Hoover, 1985; Kusnezov et al., 1990; Nosé, 1991), in which appropriate coupling to external degrees of freedom adjusts the temperature, are on firm grounds in classical mechanics and have been used successfully for equilibrium, e.g. to investigate classical spin systems, as well as for non-equilibrium, e.g. to study glass transitions.

The classical procedures fail for quantum systems and especially for identical fermions when the phase-space density is not small anymore and the effects of the Pauli principle become important. No analogue to thermostated time evolutions exists yet for quantum systems. There are few attempts to infer thermodynamic properties from dynamical simulations in quantum mechanics, some of them are given in (Kusnezov, 1993; Ohnishi and Randrup, 1993; Klakow et al., 1994; Blaise et al., 1994; Schnack and Feldmeier, 1996; Ono and Horiuchi, 1996b; Ebeling and Militzer, 1997; Ohnishi and Randrup, 1997; Ohnishi and Randrup, 1997; Schnack and Feldmeier, 1997; Schnack, 1998). Usually one performs timeaverages and relies on the ergodic assumption. The validity of this method depends of course crucially on the statistical and ergodic properties of the dynamical model. Following the mentioned articles one realizes that the matter is still under debate.

Two general aspects are discussed in detail in the following sections. One concerns the thermostatic properties of a molecular dynamics model where the attribute thermostatic refers to the characteristics of the static canonical statistical operator, which determines the quantal partition function $Z(T)=\operatorname{Tr}(\exp \{\underset{\sim}{-H} / T\})$. Once the trace is evaluated within a given model, its thermostatic properties can be deduced by standard methods like partial derivatives of $\ln Z(T)$ with respect to temperature $T$ or other parameters contained in the 
Hamilton operator $\underset{\sim}{H}$.

The other and even more important aspect, which is discussed in Sec. VB, is the dynamical behaviour of a molecular dynamics model. For example a dissipative system which is initially far from equilibrium is expected to equilibrate towards the canonical ensemble. The simulation of such a system within the model provides a crucial test of its thermodynamic properties. Often one uses an ergodicity assumption, i.e. that time-averages are equivalent to ensemble averages, which should be verified.

\section{A. Thermostatics}

The question of thermostatic properties can be condensed to the question, whether the set of model states $|Q\rangle$ is complete, i.e. able to span the many-particle Hilbert space. If that is the case the unit operator in $N$-particle space can be written as

$$
\stackrel{1}{\sim}^{(N)}=\int \mathrm{d} \mu(Q) \frac{|Q\rangle\langle Q|}{\langle Q \mid Q\rangle}
$$

where $\mathrm{d} \mu(Q)$ is a measure which depends on the parameter set $Q$ because the trial states $|Q\rangle$ are in general nonorthogonal. For fermions and gaussian wave packets the measure is derived in the following subsection.

Once the completeness is shown the thermostatic relations have to be correct, provided the trace of the partion function

$$
\begin{aligned}
Z(T) & =\operatorname{Tr}(\exp \{-\underset{\sim}{H} / T\}) \\
& =\int \mathrm{d} \mu(Q) \frac{\langle Q|\exp \{-\underset{\sim}{H} / T\}| Q\rangle}{\langle Q \mid Q\rangle} .
\end{aligned}
$$

is calculated with the trial states $|Q\rangle$ in quantum fashion.

In the case of Fermionic Molecular Dynamics (FMD) and Antisymmetrized Molecular Dynamics (AMD) (Schnack and Feldmeier, 1996; Ono et al., 1992a; Ono et al., 1992b) the antisymmetric many-body states form an overcomplete set and provide a full representation for the unit operator. As the calculation of the trace does not depend on the representation 
all thermostatic properties like Fermi-Dirac distribution, specific heat, mean energy as a function of temperature etc. ought to be correct and fully quantal using FMD or AMD trial states. The difficult task is to calculate $\langle Q|\exp \{\underset{\sim}{-\underset{\sim}{\sim}} / T\}| Q\rangle$, or $\langle Q|\underset{\sim}{\operatorname{B}} \exp \{\underset{\sim}{-\underset{\sim}{H}} / T\}| Q\rangle$, where $\underset{\sim}{\mathbb{Z}}$ is an observable. The trace integral can be evaluated by Monte-Carlo methods.

\section{Completeness relation with coherent states}

In the following it is shown that Slater determinants of coherent states span the whole Hilbert space for fermions.

Coherent states $|\vec{z}\rangle$ which are defined as the eigenstates of the harmonic oscillator destruction operator $\underset{\vec{a}}{\sim}=\sqrt{\frac{1}{2 a_{0}}} \vec{x}+i \sqrt{\frac{a_{0}}{2}} \underset{\sim}{\sim}$

$$
\underset{\vec{a}}{\sim}|\vec{z}\rangle=\vec{z}|\vec{z}\rangle, \quad \stackrel{h}{\sim}_{\mathrm{HO}}=\frac{1}{m a_{0}}\left(\underset{\vec{a}}{\sim} \stackrel{\vec{a}}{\sim}+\frac{3}{2}\right)
$$

form an overcomplete set of states. They are the gaussian states defined in Eq. (45) with $\vec{z}=\sqrt{\frac{1}{2 a_{0}}} \vec{r}+i \sqrt{\frac{a_{0}}{2}} \vec{p}=\vec{b} / \sqrt{2 a_{0}}$ for a real width parameter $a_{0}$. Their completeness relation reads in single-particle space

$$
\begin{aligned}
{\underset{1}{1}}^{(1)} & =\int \frac{\mathrm{d}^{3} \operatorname{Re} z \mathrm{~d}^{3} \operatorname{Im} z}{\pi^{3}} \frac{|\vec{z}\rangle\langle\vec{z}|}{\langle\vec{z} \mid \vec{z}\rangle} \\
& =\int \frac{\mathrm{d}^{3} r \mathrm{~d}^{3} p}{(2 \pi)^{3}} \frac{|\vec{r}, \vec{p}\rangle\langle\vec{r}, \vec{p}|}{\langle\vec{r}, \vec{p} \mid \vec{r}, \vec{p}\rangle},
\end{aligned}
$$

where $|\vec{z}\rangle$ labels the coherent states by their eigenvalue with respect to $\underset{\sim}{\sim}$, and the phase space notation $|\vec{r}, \vec{p}\rangle$ labels the states by their expectations values of coordinate and momentum operators. Coherent states are extensively discussed in (Klauder and Skagerstam, 1985).

Since we are dealing with fermions the spin degree of freedom has to be considered and consequently the resolution of unity changes to

$$
{\underset{1}{\sim}}^{(1)}=\int \frac{\mathrm{d}^{3} r \mathrm{~d}^{3} p}{(2 \pi)^{3}} \sum_{m} \frac{|q\rangle\langle q|}{\langle q \mid q\rangle},
$$

where the sum runs over the two magnetic quantum numbers $m= \pm \frac{1}{2}$ which are included in the set of parameters denoted by $q$ (see Eq. (71)). 
Proceeding one step further the unit operator in the antisymmetric part of the twoparticle Hilbert space is the antisymmetric product of two single-particle unit operators

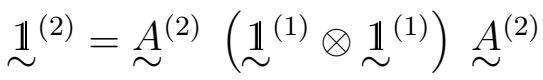

$$
\begin{aligned}
& =\frac{1}{2}\left(1-{\underset{\sim}{P}}_{12}\right)\left({\underset{\sim}{\sim}}^{(1)} \otimes{\underset{\sim}{1}}^{(1)}\right) \frac{1}{2}\left(1-{\underset{\sim}{P}}_{12}\right) \text {, }
\end{aligned}
$$

which may be expressed with antisymmetric two-particle states $\left|q_{1}, q_{2}\right\rangle_{a}$ as

$$
\begin{aligned}
& \stackrel{\mathbb{1}}{\sim}^{(2)}=\int \frac{\mathrm{d}^{3} r_{1} \mathrm{~d}^{3} p_{1}}{(2 \pi)^{3}} \sum_{m_{1}} \int \frac{\mathrm{d}^{3} r_{2} \mathrm{~d}^{3} p_{2}}{(2 \pi)^{3}} \sum_{m_{2}} \frac{\left|q_{1}, q_{2}\right\rangle_{a}\left\langle q_{1}, q_{2}\right|}{\left\langle q_{1} \mid q_{1}\right\rangle\left\langle q_{2} \mid q_{2}\right\rangle} \\
& \text { where } \quad\left|q_{1}, q_{2}\right\rangle_{a}:=\frac{1}{2}\left(\left|q_{1}\right\rangle \otimes\left|q_{2}\right\rangle-\left|q_{2}\right\rangle \otimes\left|q_{1}\right\rangle\right) .
\end{aligned}
$$

Following this line the resolution of unity in the antisymmetric part of the $N$-particle Hilbert space can be written as the projection of the $N$-particle unit operator onto the antisymmetric subspace. Be $|Q\rangle$ the unnormalized Slater determinant (70) of single-particle states $|q\rangle$

$$
|Q\rangle=\frac{1}{N !} \sum_{P} \operatorname{sgn}(P)\left(\left|q_{P(1)}\right\rangle \otimes \cdots \otimes\left|q_{P(N)}\right\rangle\right)
$$

then the unit operator is

$$
\stackrel{\mathbb{1}}{\sim}^{(A)}=\int \mathrm{d} \mu(Q) \frac{|Q\rangle\langle Q|}{\langle Q \mid Q\rangle}
$$

with a measure

$$
\mathrm{d} \mu(Q)=\langle Q \mid Q\rangle \prod_{k=1}^{N} \frac{1}{\left\langle q_{k} \mid q_{k}\right\rangle} \frac{\mathrm{d}^{3} r_{k} \mathrm{~d}^{3} p_{k}}{(2 \pi)^{3}} \sum_{m_{k}},
$$

that accounts for antisymmetrization. In a sampling where the values of $\vec{r}_{k}$ and $\vec{p}_{k}$ are chosen according to Monte-Carlo methods this measure determines the probability to find the state $|Q\rangle$ belonging to the configuration $Q=\left\{\vec{r}_{1}, \vec{p}_{1}, m_{1} ; \vec{r}_{2}, \vec{p}_{2}, m_{2} ; \ldots\right\}$ in Hilbert space. If for example two fermions with the same spin are close in $\vec{r}$ and $\vec{p}$ then this measure is small because the norm $\langle Q \mid Q\rangle=\operatorname{det}\left\{\left\langle q_{k} \mid q_{l}\right\rangle\right\}$ will be small. Eq. (151) is very useful in calculating traces by means of Monte-Carlo sampling (Ohnishi and Randrup, 1993). 
Coherent states are gaussian wave-packets with fixed width, but the real and imaginary part of the width, $a_{R}$ and $a_{I}$, may also be integrated over appropriate ranges in order to get an improved coverage of the phase space when doing the sampling.

$$
\mathbb{1}^{(1)}=\int \frac{\mathrm{d}^{3} r \mathrm{~d}^{3} p}{(2 \pi)^{3}} \sum_{m} \int_{\Omega_{R}} \frac{\mathrm{d} a_{R}}{\Omega_{R}} \int_{\Omega_{I}} \frac{\mathrm{d} a_{I}}{\Omega_{I}} \frac{|q\rangle\langle q|}{\langle q \mid q\rangle},
$$

where $\Omega_{R}$ and $\Omega_{I}$ denote the intervals the width $a=a_{R}+i a_{I}$ is integrated over

$$
\int_{\Omega_{R}} \mathrm{~d} a_{R}=\Omega_{R}, \quad \int_{\Omega_{I}} \mathrm{~d} a_{I}=\Omega_{I}
$$

Since the width $a$ in the completeness relation Eq. (146) is arbitrary, the additional integrations in Eq. (153) do not change the unit operator.

In the case of nuclear physics one has two types of fermions, so that the measure for a system with $N$ neutrons and $Z$ protons is

$$
\mathrm{d} \mu(Q)=\langle Q \mid Q\rangle \prod_{k=1}^{N} \frac{1}{\left\langle q_{k} \mid q_{k}\right\rangle} \frac{\mathrm{d}^{3} r_{k} \mathrm{~d}^{3} p_{k}}{(2 \pi)^{3}} \sum_{m_{k}} \prod_{l=N+1}^{A=N+Z} \frac{1}{\left\langle q_{l} \mid q_{l}\right\rangle} \frac{\mathrm{d}^{3} r_{l} \mathrm{~d}^{3} p_{l}}{(2 \pi)^{3}} \sum_{m_{l}} .
$$

Once the resolution of unity is given in terms of model states the partition function can be evaluated.

It is interesting to note that the norm $\langle Q \mid Q\rangle$ in the measure cancels with the norm denominator in Eq. (144). Provided the single-particle states are normalized, $\left\langle q_{k} \mid q_{k}\right\rangle=1$, and $\underset{\sim}{H}$ does not depend on the spins, the partion function looks almost classical

$$
Z(T)=\frac{2^{N}}{(2 \pi)^{3 N}} \int \mathrm{d}^{3} r_{1} \mathrm{~d}^{3} p_{1} \mathrm{~d}^{3} r_{2} \mathrm{~d}^{3} p_{2} \cdots \mathrm{d}^{3} r_{N} \mathrm{~d}^{3} p_{N}\langle Q|\exp \{\underset{\sim}{-H} / T\}| Q\rangle,
$$

except that there is an operator in the exponent. Eq. (155) is still the exact quantum expression. There is no contradiction between the fact that the hamiltonian may have a discrete energy spectrum with gaps between the levels and the fact that the parameters $\vec{r}_{k}$ and $\vec{p}_{k}$ are integrated in a continous fashion (Schnack, 1999). Only if the expectation value is moved up into the exponent things become wrong. The Pauli exclusion principle is fully taken care of by the antisymmetric state $|Q\rangle$. If two particles with equal spin are at the same point in phase space $|Q\rangle=0$ and hence $\langle Q|\exp \{\underset{\sim}{H} / T\}| Q\rangle=0$, so that forbidden states do not contribute to the partition function. 


\section{Example for many fermions}

In the following the above considerations are illustrated with the example of $N$ identical fermions in a common single-particle potential. Starting from the Hamilton operator

$$
\underset{\sim}{H}=\sum_{l=1}^{N} \underset{\sim}{h}(l), \quad \underset{\sim}{\sim}(l)=\frac{\vec{k}^{2}(l)}{2 m}+v(\underset{\sim}{\sim}(l))
$$

the mean energy of the $N$-fermion system can be derived from the partition function $Z(T)$, Eq. (144), as the derivative with respect to $T$

$$
\begin{aligned}
\langle\langle\underset{\sim}{\sim}\rangle\rangle_{\mid T} & =T^{2} \frac{\partial}{\partial T} \ln (Z(T)) \\
& =\frac{\int \mathrm{d} \mu(Q) \mathcal{W}(T) \sum_{m, n}^{N} \mathcal{O}_{n m}(T)\left[T^{2} \frac{\partial}{\partial T}\left\langle q_{m}|\exp \{-\underset{\sim}{h} / T\}| q_{n}\right\rangle\right]}{\int \mathrm{d} \mu(Q) \mathcal{W}(T)}
\end{aligned}
$$

where the two abbreviations $\mathcal{W}(T)$ and $\mathcal{O}^{-1}(T)$ are introduced as

$$
\begin{aligned}
\mathcal{W}(T) \equiv \frac{\langle Q|\exp \{\underset{\sim}{\sim} / T\}| Q\rangle}{\langle Q \mid Q\rangle} & =\frac{\operatorname{det}\left(\left\langle q_{k}|\exp \{-\underset{\sim}{h} / T\}| q_{l}\right\rangle\right)}{\operatorname{det}\left(\left\langle q_{k} \mid q_{l}\right\rangle\right)}, \\
\left(\mathcal{O}^{-1}(T)\right)_{k l} & =\left\langle q_{k}|\exp \{-\underset{\sim}{h} / T\}| q_{l}\right\rangle .
\end{aligned}
$$

For free motion, i.e. $v(\underset{\sim}{\vec{x}})=0$, the matrix elements (159) are given by:

$$
\left\langle q_{k}\left|\exp \left\{-\frac{\vec{k}^{2}}{2 m T}\right\}\right| q_{l}\right\rangle=\left(\frac{2 \pi a_{k}^{*} a_{l}}{a_{k}^{*}+a_{l}+\frac{1}{m T}}\right)^{\frac{3}{2}} \exp \left\{-\frac{\left(\vec{b}_{k}^{*}-\vec{b}_{l}\right)^{2}}{2\left(a_{k}^{*}+a_{l}+\frac{1}{m T}\right)}\right\} \text {. }
$$

For the special case of the three-dimensional harmonic oscillator, i.e. $v(\underset{\sim}{\vec{x}})=\frac{1}{2} m \omega^{2} \stackrel{\vec{x}}{\sim}^{2}$, the matrix elements can easily be calculated if one sets all $a_{k}=a_{0}=1 /(m \omega)$ (Schnack, 1996):

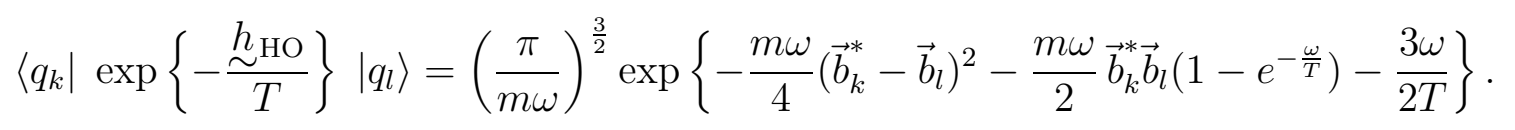

For other potentials the matrix elements (159) assume a more complicated form.

Figure 7 shows the result of a Metropolis integration (Metropolis et al., 1953) over $\vec{r}_{k}$ and $\vec{p}_{k}$ of Eq. (157) for a system of four identical fermions with equal spins in a harmonic oscillator by open circles. The solid line displays the results when the traces are calculated by discrete sums over eigenstates of the many-body Hamilton operator $\underset{\sim}{H_{\mathrm{HO}}}$ (Schmidt and Schnack, 


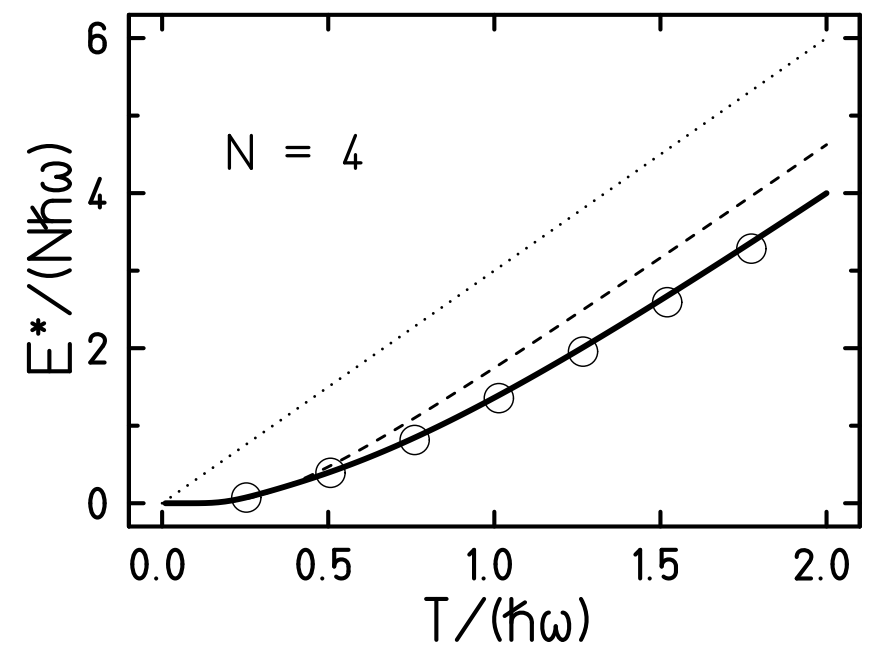

FIG. 7. Excitation energy as a function of temperature, calculated with antisymmetrized gaussian states (circles) or with eigenfunctions of the harmonic oscillator (solid line). The dotted line shows the classical result $E^{*} / N=3 T$ and the dashed line the result of quantum Boltzmann statistics.

1998). For comparison the classical dependence is shown as a dotted line. The dashed line represents the result for the quantum Boltzmann case for distinguishable particles, were the trial state in Eq. (155) is a direct product of single-particle states. In both quantum cases the specific heat is vanishing at $T=0$ because of the finite energy gap between ground state and first excited state.

As expected from the general argument that traces are independent of the representation, provided one uses a complete set, the numerical Metropolis integration with a sampling of $10^{6}$ points in the 24 -dimensional phase space of the continuous parameters $\vec{r}_{k}, \vec{p}_{k}$, which specify the Slater determinants $|Q\rangle$, gives the same result as summing over eigenstates of the many-body hamiltonian. The continous integration is not in contradiction with Fermi statistics and the discrete spectrum of the hamiltonian, and the classically looking Eq. (155) is fully quantal. 
1. The thermostatic properties of a model ought to be correct, if the set of model states $|Q\rangle$ is complete, i.e. able to span the many-particle Hilbert space.

2. In FMD and AMD the antisymmetric many-body states of single-particle gaussian wave packets form an overcomplete set and provide a full representation for the unit operator. 


\section{B. Thermodynamics}

In molecular dynamics the time evolution as given by the time-dependent variational principle, without a collision term or quantum branching, is deterministic. Given a state $\left|Q\left(t_{0}\right)\right\rangle$ at an initial time $t_{0}$ the system is described by the pure state $|Q(t)\rangle$ at all earlier and later times. Therefore, like with the exact solution of the Schrödinger equation, thermal properties have to be obtained by coarse graining or time averaging.

In this section time averaging is compared with the canonical statistical ensemble for a fermion system. If the system is ergodic both are equivalent and equilibrium properties can be evaluated by molecular dynamics simulations. For this the ergodic ensemble is defined by the statistical operator $\underset{\sim}{\operatorname{erg} \text { as }}$

$$
\underset{\sim}{R} \operatorname{erg}:=\lim _{t_{2} \rightarrow \infty} \frac{1}{\left(t_{2}-t_{1}\right)} \int_{t_{1}}^{t_{2}} \mathrm{~d} t \frac{|Q(t)\rangle\langle Q(t)|}{\langle Q(t) \mid Q(t)\rangle}
$$

Herewith the ergodic mean of an operator $\underset{\sim}{\sim}$ is given by

$$
{\overline{\langle\underset{\sim}{\sim}\rangle}}_{|\underset{\sim}{\sim}\rangle}:=\operatorname{Tr}(\underset{\sim}{R} \operatorname{erg} \underset{\sim}{B})=\lim _{t_{2} \rightarrow \infty} \frac{1}{\left(t_{2}-t_{1}\right)} \int_{t_{1}}^{t_{2}} \mathrm{~d} t \frac{\langle Q(t)|\underset{\sim}{B}| Q(t)\rangle}{\langle Q(t) \mid Q(t)\rangle} .
$$

If the ergodic assumption is fulfilled, the statistical operator $\underset{\sim}{R} \operatorname{erg}$ should depend only on $\langle\underset{\sim}{\sim}\rangle$, which is actually a constant of motion. Therefore the notation with the condition " $\langle\underset{\sim}{H}\rangle "$ in Eq. (163) is used.

Expectation values are well-defined with Eq. (163) so that one can easily calculate extensive quantities like the excitation energy. But it is not obvious how an intensive thermodynamical quantity, such as the temperature, might be extracted from deterministic molecular dynamics with wave packets.

In classical mechanics with momentum-independent interactions the partition function

$$
\begin{aligned}
& Z_{\text {classical }}(T)=\int \prod_{k=1}^{N} \frac{\mathrm{d}^{3} r_{k} \mathrm{~d}^{3} p_{k}}{(2 \pi)^{3}} \exp \left\{-\frac{1}{T} \mathcal{H}_{\text {classical }}\left(\vec{r}_{1}, \vec{p}_{1}, \cdots\right)\right\} \\
= & \prod_{k=1}^{N} \int \mathrm{d}^{3} p_{k} \exp \left\{-\frac{1}{T} \frac{\vec{p}_{k}^{2}}{2 m_{k}}\right\} \cdot \int \prod_{l=1}^{N} \frac{\mathrm{d}^{3} r_{l}}{(2 \pi)^{3}} \exp \left\{-\frac{1}{T} \mathcal{V}\left(\vec{r}_{1}, \vec{r}_{2}, \cdots\right)\right\}
\end{aligned}
$$

is a product of a term with the kinetic energy and a term containing the interactions. Therefore, a fit of the momentum distribution with a Boltzmann distribution, or the equipartition 
theorem, can be used to determine the temperature $T$. For example, in simple formulations of the Nosé-Hoover thermostat (Hoover, 1985; Kusnezov et al., 1990; Nosé, 1991) the equipartition theorem serves as basic ingredient.

In the quantum case Eq. (144) has to be employed which does not show this factorization. For an interacting finite system one cannot, in analogy to the Boltzmann case, fit a Fermi function to the momentum distribution to determine the temperature. An example is the ground state of a nucleus for which the momentum distribution has a smeared out Fermi edge due to the finite size and the two-body interaction and not because of temperature.

\section{Ergodic ensemble of fermions in a harmonic oscillator}

In this section the ideal gas of fermions in a common one-dimensional harmonic oscillator potential is used for demonstration. The hamiltonian $\underset{\sim}{H_{\mathrm{HO}}}$ is written in second quantization with the fermion creation operator $\underset{\sim}{\stackrel{c}{\sim}}$ (which creates a fermion in the $n$-th single-particle eigenstate of $\underset{\sim}{h} \mathrm{HO}$ ) as

$$
{\underset{\mathrm{H}}{\mathrm{HO}}}_{\mathrm{HO}}=\sum_{l=1}^{N} \underset{\mathrm{h}}{\sim} \mathrm{HO}(l)=\sum_{l=1}^{N}\left(\frac{\vec{k}^{2}(l)}{2 m}+\frac{1}{2} m \omega^{2} \stackrel{\vec{x}}{\sim}^{2}(l)\right)=\omega \sum_{n=0}^{\infty}\left(n+\frac{1}{2}\right) \stackrel{c^{+}}{\sim} \stackrel{c}{\sim}_{n}
$$

and the trial state $|Q(t)\rangle$ describes four fermions with equal spins. To test the fermionic nature of the dynamical evolution the ergodic ensemble averages of the occupation numbers,

${\overline{\left\langle\underset{\sim}{c_{n}^{+} \underset{\sim}{\sim}{ }_{n}}\right\rangle_{\mid}}}_{\langle\underset{\sim}{\sim}\rangle}$, see Eq. (163), are evaluated and compared with $\left\langle\left\langle\underset{\sim}{\sim}{ }_{n}^{+} \underset{\sim}{\sim}{ }_{n}\right\rangle\right\rangle_{T_{T}}$ of the canonical ensemble discussed in the previous section.

The occupation numbers, which range from 0 to 1 for fermions, are chosen on purpose to make clear from the beginning, that the equations of motion for the parameters $Q(t)=$ $\left\{\vec{r}_{1}(t), \vec{p}_{1}(t), \cdots\right\}$, as given by Eq. (22), might be generalized Hamilton equations, but the observables always have to be calculated with the quantum state $|Q(t)\rangle$. If $\vec{r}_{k}(t)$ and $\vec{p}_{k}(t)$ were taken as the particle coordinates in a classical picture, the question for the mean occupation number of the $n$-th eigenstate of the harmonic oscillator could even not be posed.

As pointed out already, in Fermionic Molecular Dynamics (FMD) the time evolution of gaussian wave packets in a common oscillator is exact, and thus the occupation probabilities 
of the eigenstates of the Hamilton operator do not change in time. In order to equilibrate the system a repulsive short-range two-body interaction is introduced. The strength of the interaction is chosen weak enough so that the ideal gas picture is approximately still valid.

In the initial state $\left|Q\left(t_{0}\right)\right\rangle$, which is far from equilibrium, three wave packets with a width of $a\left(t_{0}\right)=1 / m \omega$ are put close to the origin at $x\left(t_{0}\right)=(-d, 0, d)$ with $d=0.5 / \sqrt{m \omega}$, while the fourth packet is pulled away from the center.

Figure 8 gives an impression of the chaotic time dependence of $\left\langle Q(t)\left|\underset{\sim}{\stackrel{+}{\sim}} \underset{\sim}{\sim}{ }_{n}\right| Q(t)\right\rangle$ for $n=0,3$ and 6 .

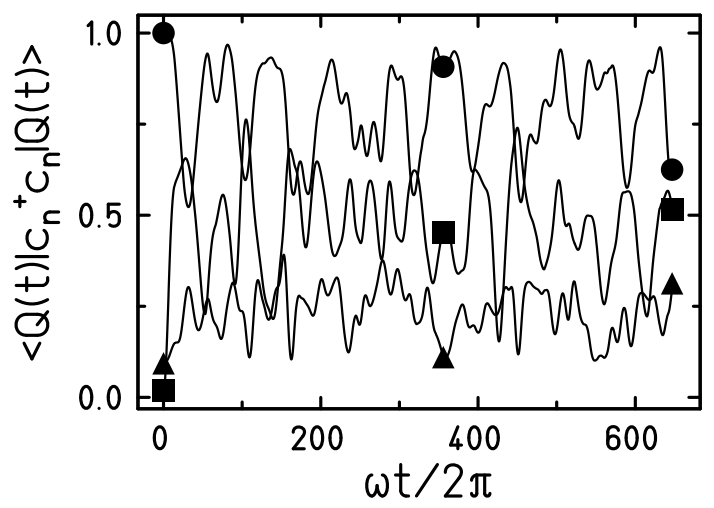

FIG. 8. Occupation probabilities versus time $-n=0$ : circles, $n=3$ : squares, $n=6:$ triangles.

The result of time averaging is seen in Fig. 9 (symbols) for four different initial displacements which correspond to four different excitation energies of the fermion system. To each case we assign a canonical ensemble which has the same mean energy, i.e. $E^{*}={\overline{\left\langle\underset{\sim}{H} \mathrm{HO}-E_{0}\right\rangle_{\mid}}}_{\langle\underset{\sim}{\sim}\rangle}=\left\langle\left\langle\underset{\sim}{H_{\mathrm{HO}}}-E_{0}\right\rangle\right\rangle_{\left.\right|_{T}}$. The solid lines in Fig. 9 show the corresponding distributions of occupation probabilities for these canonical ensembles. Their temperatures $T$ are also quoted in the figure. The one to one correspondence between the occupation probabilities of the ergodic ensemble and the ones of the canonical ensemble, which has the same mean energy $\langle\underset{\sim}{\sim}\rangle$ as the pure state, demonstrates that the system is ergodic and that the fermion molecular dynamics trajectory covers the many-body phase space according to Fermi-Dirac statistics. 


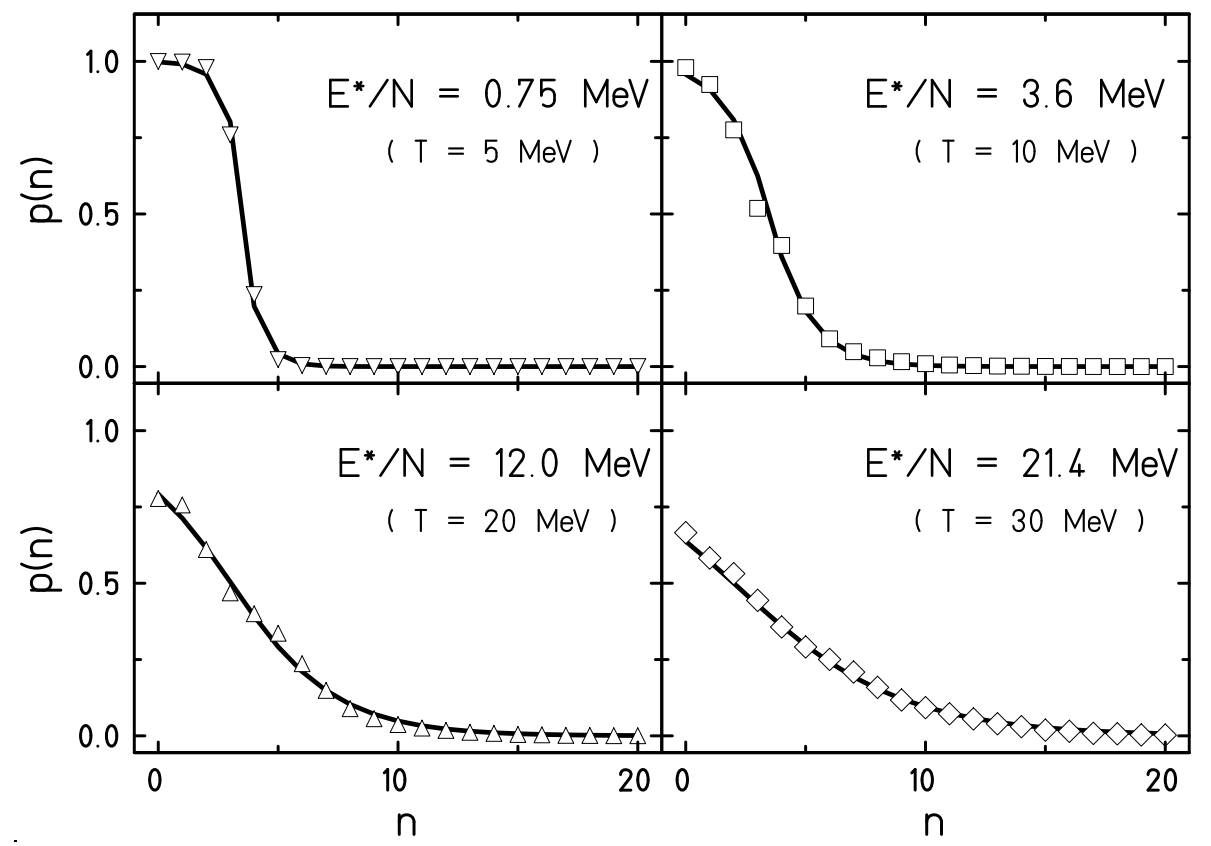

FIG. 9. Occupation probabilities calculated in the ergodic ensemble $\overline{\left\langle\underset{\sim}{\stackrel{c}{{ }_{n}^{+}} \underset{\sim}{c}{ }^{n}}\right\rangle}|\underset{\sim}{\sim}\rangle$

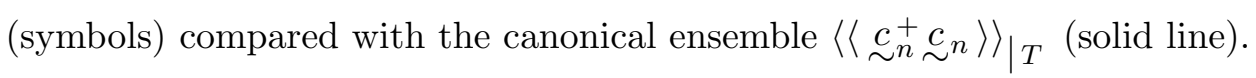

This result is not trivial because, firstly, the system is very small, consisting of only four particles, and secondly, the equations of motion are approximated by FMD.

\section{Describing the system with fixed-width trial states}

As explained already in Sec. V A, both type of trial states with dynamical and fixed widths span the whole Hilbert space and thus the thermostatic properties are the same. Reducing the degrees of freedom by keeping all width parameters at a fixed value $a_{0}$ leads however to a different dynamical behavior as discussed in Sec. III A. The equations of motion are no longer exact solutions for the case of free motion. In a common oscillator the exact solution is obtained only if all $a_{l}=1 /(m \omega)$ because $d a_{l} / d t$ is zero in that special case, see Sec. IV A 3. If the width has a different value, spurious scattering occurs like in the case of free motion whenever two particles come too close in phase space.

The left hand part of Fig. 10 displays for the very same system as in the previous 
section the result of the time evolution but without the randomizing two-body interaction. If the widths are chosen to be $a_{l}=1 /(m \omega)$, the resulting exact time evolution is just a unitary transformation in the one-body space and the occupation probabilities are stationary (circles). But if the widths are taken as $a_{l}=1.2 /(m \omega)$ the occupation probabilities change in time and the spurious scatterings equilibrate the system even without an interaction. The right hand part of Fig. 10 shows the mean occupation probabilities when the interaction is switched on (triangles). Apparently the nature of the randomizing force is not relevant, it may even be a spurious force which originates from a too restricted trial state.
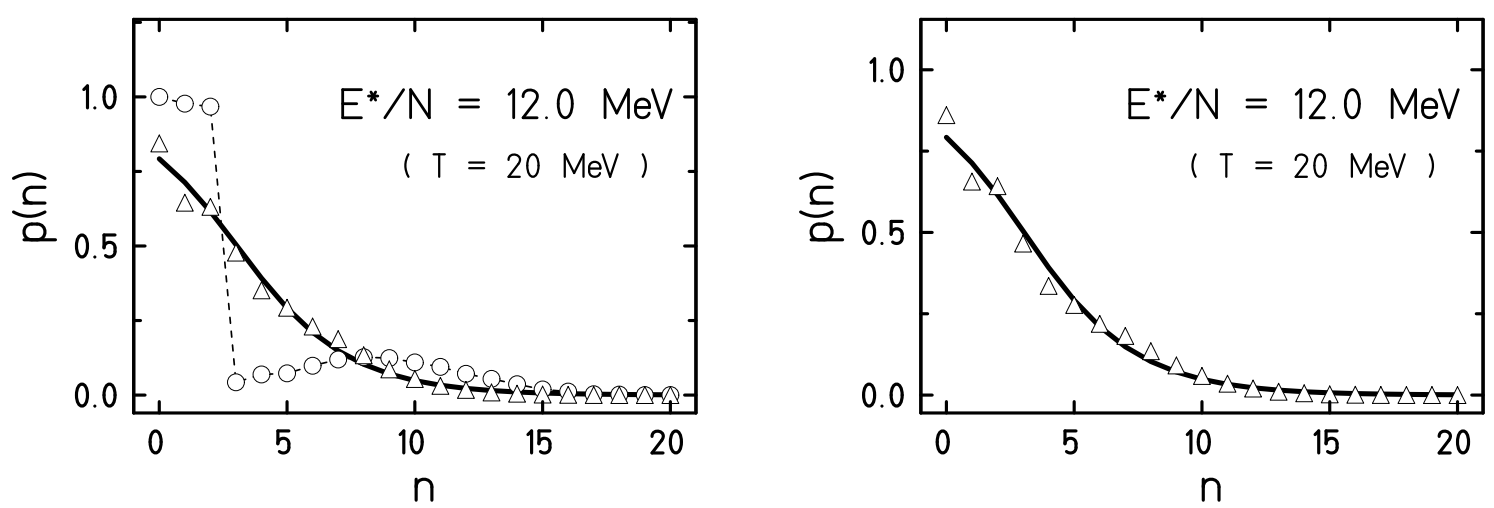

FIG. 10. Occupation probabilities calculated in the ergodic ensemble using trial states with fixed widths (symbols) compared to those calculated in the canonical ensemble (solid line). L.h.s.: Without interaction, circles: $a_{l}=1 /(m \omega)$, triangles: $a_{l}=1.2 /(m \omega)$. R.h.s.: With interaction and $a_{l}=1.2 /(m \omega)$.

In Antisymmetrized Molecular Dynamics (AMD) (Ono et al., 1992a; Ono et al., 1992b) trial states with time-independent widths are used, and as expected from the above simple example the thermodynamic properties of the model comply with Fermi-Dirac statistics. It would be interesting to see how collision term and branching influence the dynamical statistical properties of AMD. As the Pauli-blocking prescription is consistent with the AMD state we expect a faster equilibration due to the additional randomization. 


\section{Canonical and ergodic ensemble for distinguishable particles}

To complete the discussion in this section the fermions are replaced by distinguishable particles, i.e. the antisymmetrized many-body state is replaced by a product state of gaussian wave packets. In this case the relation between temperature $T$ and excitation energy $E^{*} / N$ is known and given by $E^{*} / N=\frac{1}{2} \omega\left[\operatorname{coth}\left(\frac{\omega}{2 T}\right)-1\right]$.

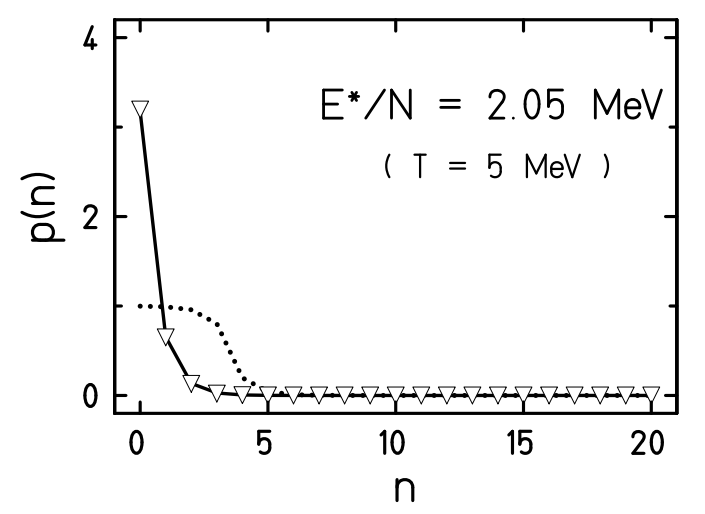

FIG. 11. Mean occupation numbers for a product state (Boltzmann statistics). Ergodic ensemble (triangles), canonical ensemble (solid line), both for an excitation energy of $E^{*} / N=2.05 \mathrm{MeV}$ which corresponds to a temperature $T=5 \mathrm{MeV}$ in the canonical ensemble. The dotted line shows the result for Fermi-Dirac statistics at the same temperature.

The ergodic ensemble is again investigated at different energies and compared with the corresponding canonical ensembles with the same mean energies (Schnack and Feldmeier, 1996; Feldmeier and Schnack, 1997). The time evolution of the system exhibits also for this case ergodic behaviour at all excitation energies. As an example Fig. 11 is showing the case of $E^{*} / N=2.05 \mathrm{MeV}$ (which corresponds to $T=5 \mathrm{MeV}$ ) after a time averaging of about 2000 periods. The ergodic ensemble (triangles) and the Boltzmann canonical ensemble (solid line) are the same within the size of the symbols. This means that the ergodic ensemble is again equivalent to the quantum canonical ensemble and not to the classical one, because for $E^{*} / N=2.05 \mathrm{MeV}$ and $T=5 \mathrm{MeV}$ one is still in the quantal regime according to the 
relation given above. The classical relation $E^{*} / N=T$ for the one-dimensional oscillator holds only for $E^{*} / N \gg \omega$ (here $\omega=8 \mathrm{MeV}$ ).

However, since distinguishable particles are not affected by the Pauli principle, the occupation numbers for the many-body ground state look quite different from those of the Fermi-Dirac distribution at the same temperature (dotted line in Fig. 11).

\section{Resumé}

When discussing statistical properties of molecular dynamics with gaussian wave-packets (coherent states) one should always keep in mind that any observable or statistical weight has to be calculated with the trial state according to quantum mechanics. One should not fall into a completely classical approach, misled by the "classical" appearance of equations of motion or phase space integrals, which is due to the representation of the coherent states in terms of $\vec{r}_{k}$ and $\vec{p}_{k}$.

Statistical properties of molecular dynamics for fermions can be deduced from simulations of equilibrium situations but due to quantum effects a measure for intensive quantities like temperature is not readily available. 


\section{Thermal properties of interacting systems by time averaging}

In the previous section only noninteracting or weakly interacting systems are considered for which partition functions can often be calculated because the Hamilton operator is a one-body operator. If the interaction between the particles is strong enough, for example attractive enough to form self-bound many-body systems, the ideal gas picture is no longer valid and solid and liquid phases appear besides the vapor phase. Here the partition function $Z(T)$ cannot be evaluated analytically anymore because it would amount to solving the full interacting many-body problem $\underset{\sim}{H}\left|\Psi_{n}\right\rangle=E_{n}\left|\Psi_{n}\right\rangle$ in the desired range of energies.

Take for example a fermion system in a large spherical container with repulsive walls. At zero temperature the lowest eigenstate $\left|\Psi_{n=1}\right\rangle$ describes a self-bound system in its internal ground state located at the center of the container. With increasing energies not only internal excitations and c.m. motion of this drop occur but there is also the possibility to have two or more bound objects which are separated from each other and surrounded by vapor. They can be in different internal excitations with various c.m. energies and vapor energies, all adding up to the total eigenenergy $E_{n}$. This means that the quantum number $n$ enumerates not only the excited eigenstates but also the c.m. degrees of freedom, the partition into different drops, and the fermion vapor state. The number of eigenstates in an energy interval increases rapidly with excitation energy.

In principle one can deduce all thermodynamic properties from the level density, but it is obvious that for those complex and highly correlated states $\left|\Psi_{n}\right\rangle$ it is impossible to solve the eigenvalue problem, neither analytically nor numerically. Therefore one tries to simulate correlated many-body systems by means of molecular dynamics (which describes the time evolution in an approximate way) and replaces the ensemble average by a time average. 


\section{FMD - the nuclear caloric curve}

As discussed in the section VB it can be shown that even a small system with only a few particles distributed over two different harmonic oscillator potentials is ergodic. So one can use one subsystem, for which the relation between excitation energy and temperature is known, as a thermometer to determine the temperature of the other. This idea has been used in FMD simulations of phase transitions in nuclei where the ideal Fermi gas picture does not apply because the nucleons are interacting by strong two-body forces. The construction of a thermometer is necessary because the temperature cannot be determined from the momentum distribution (Schnack and Feldmeier, 1997). In an interacting small fermion system the Fermi-edge is broadened not only due to temperature but also due to the finite size of the system and two-body correlations.

A thermostat (large heat bath coupled to the system) is not advisable because phase transitions in small systems are recognized best in a micro-canonical situation where the energy distribution is within a narrow energy range and variations of the level density $\rho(E)$,

which indicate a phase transition, are not blurred by the Boltzmann factor $e^{-E / T}$ of the canonical ensemble.

The thermometer consists of a quantum system of distinguishable particles which move in a common harmonic oscillator potential and interact with the nucleons. The nucleus itself is confined by a wide harmonic oscillator potential which serves as a containment. This is an important part of the setup because it keeps the evaporated nucleons (vapor) in the vicinity of the remaining liquid drop so that equilibration with the surrounding vapor can take place. The coupling between nucleons and thermometer particles is chosen to be weak, repulsive and of short range. It has to be as weak as possible in order not to influence the nuclear system too much. On the other hand it has to be strong enough to allow for reasonable equilibration times.

The determination of the relation between excitation energy $E^{*}$ and temperature $T$ (caloric curve) is done by time-averaging of the energy of the nucleonic system as well 
as of the thermometer over a long period according to Eq. (163). The time-averaged energy of the thermometer $E_{t h}$ determines the temperature $T$ through the known relation $E_{t h} / N_{t h}=\frac{3}{2} \omega_{t h} \operatorname{coth}\left(\omega_{t h} / 2 T\right)$ for an ideal gas of distinguishable particles in a common harmonic oscillator potential with frequency $\omega_{t h}$ (quantum Boltzmann statistics).
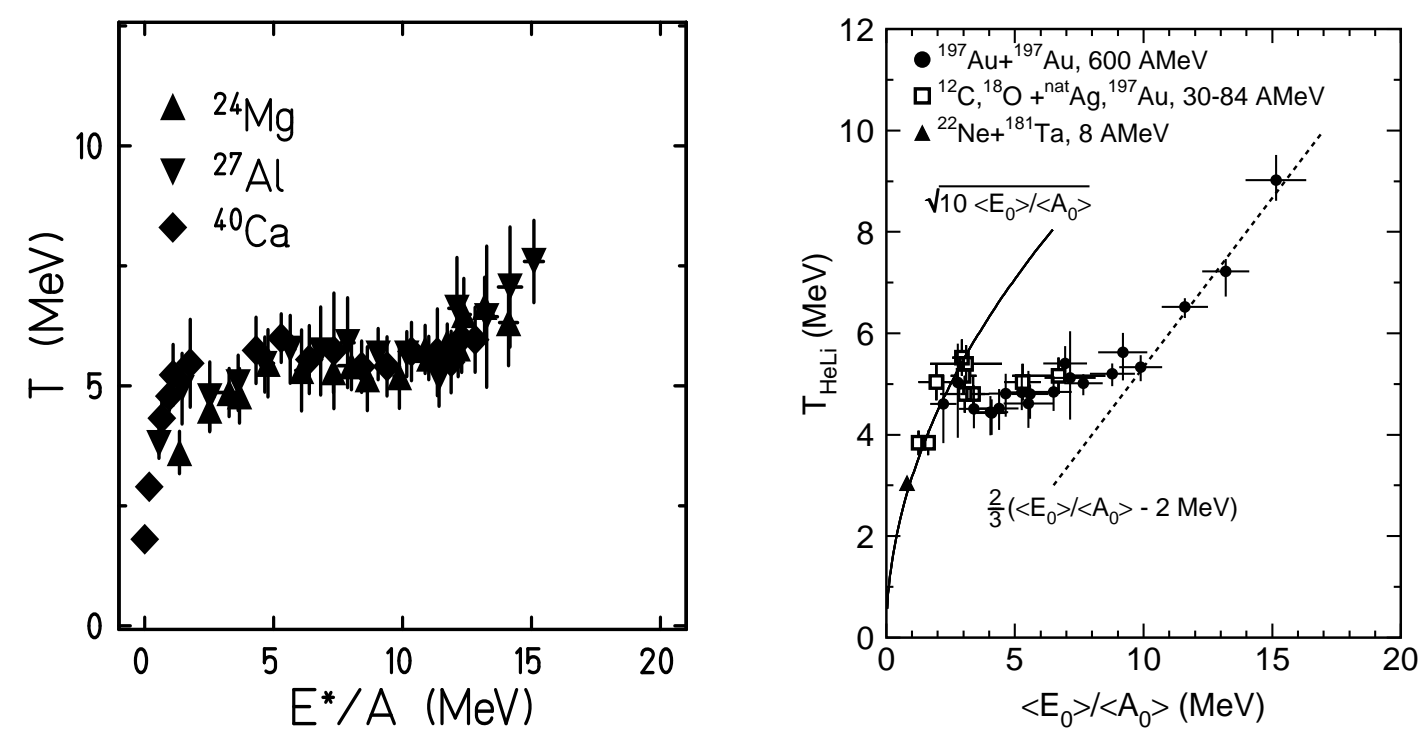

FIG. 12. Caloric curve, 1.h.s.: of ${ }^{24} \mathrm{Mg},{ }^{27} \mathrm{Al}$ and ${ }^{40} \mathrm{Ca}$ at $\hbar \omega=1 \mathrm{MeV}$, from (Schnack and Feldmeier, 1997), r.h.s.: as determined by the Aladin group from the decay of spectator nuclei, from (Pochodzalla et al., 1995).

The resulting caloric curves for the nuclei ${ }^{24} \mathrm{Mg},{ }^{27} \mathrm{Al}$ and ${ }^{40} \mathrm{Ca}$ are displayed on the left hand side of Fig. 12. All caloric curves clearly exhibit three different parts. Beginning at small excitation energies the temperature rises steeply with increasing energy as expected for a Fermi gas in the shell model. There the nucleons remain bound in the excited nucleus which behaves like a drop of liquid. At an excitation energy of $3 \mathrm{MeV}$ per nucleon the curve flattens and stays almost constant up to about $11 \mathrm{MeV}$. This plateau at $T \approx 5$ to $6 \mathrm{MeV}$ marks the coexistence phase where at low excitation energy one big drop is surrounded with low density vapor. With increasing energy the drop disolves more and more into vapor until all nucleons are unbound and the system has reached the vapor phase. The latent heat is 
hence about $8 \mathrm{MeV}$ at a pressure which is estimated to be close to zero.

The caloric curve shown on the l.h.s. of Fig. 12 has a striking similarity with the caloric curve determined experimentally from the fragmentation of colliding nuclei by the ALADIN group (Pochodzalla et al., 1995; Pochodzalla, 1997). Their results are displayed on the r.h.s. of the same figure. The position and the extension of the plateau agree quite well with the FMD calculation. Nevertheless, there are important differences between the experimental setup and the one used in the simulations. For further details see (Pochodzalla, 1997; Schnack and Feldmeier, 1997).

\section{Phase transitions of hydrogen plasma}

Hydrogen plasma under extreme conditions, high temperature or pressure is of great current interest, since it shows new structural, dynamical and electronic properties like orientational ordering, pressure induced metallization or changes in the vibronic spectra (Hemley and Mao, 1992; Klakow et al., 1994; Knaup et al., 1999).

One model employed in this context, is called wave-packet molecular dynamics. It is using gaussian packets with time-dependent widths and a Pauli potential, which is derived from the antisymmetrization of pairs of nucleons, see Sec. IV C and (Klakow et al., 1994). 256 protons and 256 electrons are distributed in a cubic box with periodic continuation in all directions. Since the protons are classical their temperature is simply given by their kinetic energy via the equipartition theorem.

The equations of motion are followed over $6 \cdot 10^{-14} \mathrm{~s}$. One observable which is sampled over this time period is the proton pair distribution function $g_{p p}(r)$ which is related to the probability of finding two particles at the distance $r$. The proton pair distribution reveals details about the binding and short range correlations in the system. In Fig. 13 the pair distribution function $g_{p p}(r)$ is plotted for two temperatures. The peak at $r=1.3$ a.u. signals that the protons are bound in $\mathrm{H}_{2}$ molecules. The authors find that due to medium effects this bond length is shifted to a smaller value compared to the free one which is $r=1.47$ a.u. 


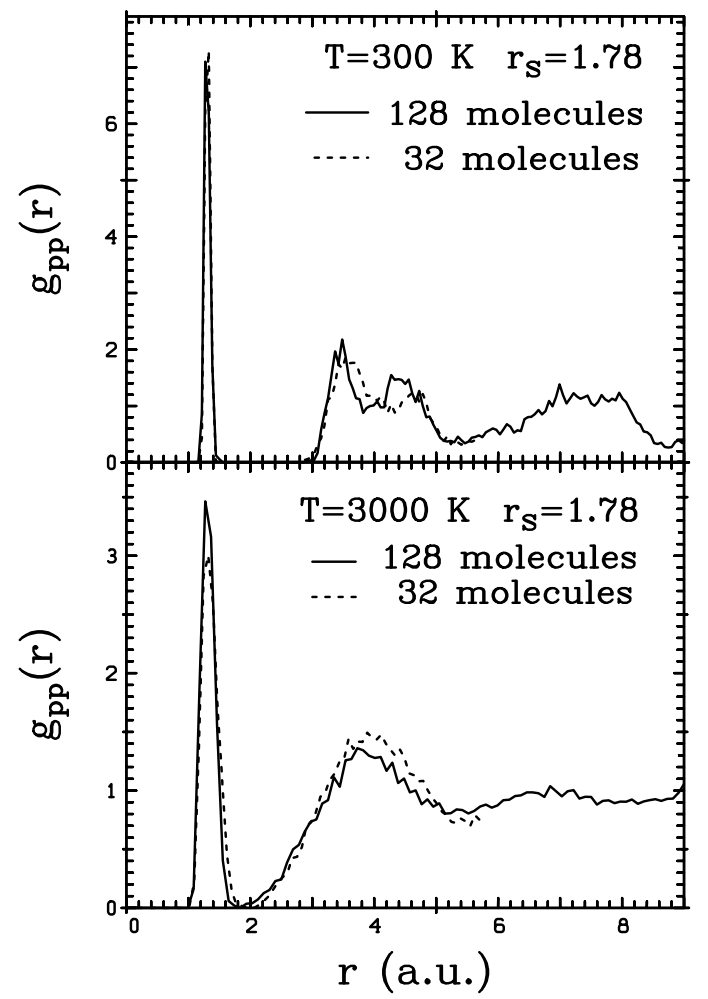

FIG. 13. The proton pair distribution function $g_{p p}(r)$ at $T=300 \mathrm{~K}$ (top) and $T=3000 \mathrm{~K}$ (bottom). The solid line shows the simulation with 128 molecules, the dashed one with 32 molecules. Figure provided by P.-G. Reinhardt.

in their model. At $T=300 \mathrm{~K}$ pronounced peaks can be seen around $r=4$ a.u. which indicate a solid structure of the $\mathrm{H}_{2}$ molecules. That these peaks are smoother at the higher temperature is interpreted as a signal that the system is now in the liquid phase.

The authors also find that wave-packet molecular dynamics is very efficient for the discussed problem and faster than the Car-Parrinello method (Car and Parrinello, 1985) while providing comparable results (Klakow et al., 1994). 


\section{ACKNOWLEDGMENTS}

For supplying us with references or figures we would like to thank S. Bass, M. Belkacem, Ph. Chomaz, P. Danielewicz, W. Ebeling, O. Knospe, Tomoyuki Maruyama, A. Ono, J. Randrup, E. Rass, P.-G. Reinhard, U. Saalmann, R. Schmidt and H. Stöcker. We also thank R. Roth for carefully reading the manuscript. 


\section{REFERENCES}

Aichelin J., and G. Bertsch, 1985, "Numerical simulation of medium energy heavy ion reactions," Phys. Rev. C 53, 1730.

Aichelin J., and H. Stöcker, 1986, "Quantum Molecular Dynamics - a novel approach to $N$-body correlations in heavy ion collisions," Phys. Lett. B 176, 14.

Aichelin J., A. Rosenhauer, G. Peilert, H. Stöcker, and W. Greiner, 1987, "Importance of momentum-dependent interactions for the extraction of the nuclear equation of state from high-energy heavy-ion collisions," Phys. Rev. Lett. 58, 1926.

Aichelin J., 1991, "Quantum Molecular Dynamics - a dynamical microscopic N-body approach to investigate fragment formation and the nuclear equation of state in heavy ion collisions," Phys. Rep. 202, 233.

Arnol'd V.I., 1989, Mathematical Methods of Classical Mechanics, (Springer-Verlag, Berlin).

Balian R., and M. Veneroni, 1988, "Static and dynamic variational principles for expectation values of observables," Ann. Phys. (N.Y.) 187, 29.

Balian R., and M. Veneroni, 1992, "Correlations and fluctuations in static and dynamic mean-field approaches," Ann. Phys. (N.Y.) 216, 351.

Bauhoff W., E. Caurier, B. Grammaticos, and M. Płoszajczak, 1985, ”Description of lightion collisions in the time-dependent cluster model," Phys. Rev. C 32, 1915.

Bass S.A., M. Belkacem, M. Bleicher, M. Brandstetter, L. Bravina, C. Ernst, L. Gerland, M. Hofmann, S. Hofmann, J. Konopka, G. Mao, L. Neise, S. Soff, C. Spieles, H. Weber, L.A. Winckelmann, H. Stöcker, W. Greiner, Ch. Hartnack, J. Aichelin, and N. Amelin, 1998, "Microscopic models for ultrarelativistic heavy ion collisions," Prog. Part. Nucl. Phys. 41, 225.

Belkacem M., M. Brandstetter, S.A. Bass, M. Bleicher, L. Bravina, M.I. Gorenstein, 
J. Konopka, L. Neise, C. Spieles, S. Soff, H. Weber, H. Stöcker, and W. Greiner, 1998, "Equation of state, spectra and composition of hot and dense infinite hadronic matter in a microscopic transport model," Phys. Rev. C 58, 1727.

Bertsch G.F., and S. Das Gupta, 1988, "A guide to microscopic models for intermediate energy heavy ion collisions," Phys. Rep. 160, 189.

Blaise P., P. Durand, and O. Henri-Rousseau, 1994, "Irreversible evolution towards equilibrium of coupled quantum harmonic oscillators. A coarse grained approach," Physica A $209,51$.

Boal D.H., and J.N. Glosli, 1988, "Quasiparticle model for nuclear dynamics studies: Ground state properties," Phys. Rev. C 38, 1870.

Bodmer A.R., and C.N. Panos, 1977, "Classical microscopic calculations of high-energy collisions of heavy ions," Phys. Rev. C 15, 1342.

Bodmer A.R., C.N. Panos, and A.D. MacKellar, 1980, "Classical-equations-of-motion calculations of high-energy heavy-ion collisions," Phys. Rev. C 22, 1025.

Bondorf J.P., D. Idier, and I.N. Mishustin, 1995, "Self-organization in expanding nuclear matter," Phys. Lett. B 359, 261.

Broeckhove J., L. Lathouwers, E. Kesteloot, and P. van Leuven, 1988, "On the equivalence of the time-dependent variational principles," Chem. Phys. Lett. 149, 547.

Broeckhove J., L. Lathouwers, and P. van Leuven, 1989, "Time-dependent variational principles and conservation laws in wavepacket dynamics," J. Phys. A: Math. Gen. 22, 4395.

Car R., and M. Parrinello, 1985, "Unified approach for molecular dynamics and density functional theory," Phys. Rev. Lett. 55, 2471.

Caurier E., B. Grammaticos, and T. Sami, 1982, "The time-dependent cluster model," Phys. Lett. B 109, 150. 
Chiba S., O. Iwamoto, T. Fukahori, K. Niita, T. Maruyama, T. Maruyama, and A. Iwamoto, 1996, "Analysis of proton-induced fragment production cross-sections by the quantum molecular dynamis plus statistical decay model," Phys. Rev. C 54, 285.

Chomaz, Ph., M.Colonna, and A.Guarnera, 1996, "Spinodal deomposition of atomic nuclei," in Advances in Nuclear Dynamics 2, edited by B. Arruada and G. Westfall, (Kluwer Academic/Plenum).

Colonna M., and Ph. Chomaz, 1998, "Spinodal decomposition in nuclear molecular dynamics," Phys. Lett. B 436, 1.

Davies K.T.R., K.R.S. Devi, S.E. Koonin, and M.R. Strayer, 1985, "TDHF calculations of heavy-ion collisions," in Treatise of Heavy-Ion Science, Vol 3, edited by D.A. Bromley, (Plenum, New York), p. 3.

Dorso C., S. Duarte, and J. Randrup, 1987, "Classical simulation of the Fermi gas," Phys. Lett. B 188, 287.

Dorso C., and J. Randrup, 1987, "Classical simulation of nuclear systems," Phys. Lett. B $\mathbf{2 1 5}, 611$.

Dorso C., and J. Randrup, 1989, "Quasi-classical simulation of nuclear dynamics. Phase evolutuion of disassembling nuclei," Phys. Lett. B 232, 29.

Drożdż S., J. Okołowicz, and M. Płoszajczak, 1982, "The time-dependent cluster theory application to the $\alpha-\alpha$ collision," Phys. Lett. B 109, 145.

Drożdż S., M. Płoszajczak, and E. Caurier, 1986, "Variational approach to the Schrödinger dynamics in the Klauder's continuous representations," Ann. Phys. 171, 108.

Ebeling W., A. Förster, and V.Yu. Podlipchuk, 1996, "Quantum wave-packets simulation of ionization processes in dense plasmas," Phys. Lett. A 218, 297.

Ebeling W., and B. Militzer, 1997, "Quantum molecular dynamics of partially ionized plas- 
mas," Phys. Lett. A 226, 298.

Ebeling W., and F. Schautz, 1997, "Many particle simulations of the quantum electron gas using momentum-dependent potentials," Phys. Rev. E 56, 3498.

Feldmeier H., 1990, "Fermionic molecular dynamics," Nucl. Phys. A 515, 147.

Feldmeier H., K. Bieler, and J. Schnack, 1995, "Fermionic molecular dynamics for ground states and collisions of nuclei," Nucl. Phys. A 586, 493.

Feldmeier H., and J. Schnack, 1997, "Fermionic molecular dynamics," Prog. Part. Nucl. Phys. 39, 393.

Feldmeier H., T. Neff, R. Roth, and J. Schnack, 1998, "A unitary correlation operator method," Nucl. Phys. A 632, 61.

J. Frenkel J., 1934, Wave mechanics, Advanced theory, (Claredon Press, Oxford), p 235.

Gaitanos T., C. Fuchs, and H.H. Wolter, 1999, "Heavy ion collisions with nonequilibrium Dirac-Brueckner mean fields," Nucl. Phys. A 650, 97.

Goeke K., and P.G. Reinhard, 1982, Eds., Time-dependend Hartree-Fock and beyond, Lecture Notes in Physics 171.

Griffin J.J., P.C. Lichtner, and M. Dworzecka, 1980, "Time-dependent S-matrix HartreeFock theory of complex reactions," Phys. Rev. C 21, 1351.

Gerjuoy E., A.R.P. Rau, and Larry Spruch, 1983, "A unified formulation of the construction of variational principles," Rev. Mod. Phys. 55, 725.

Hartnack C., L. Zhuxia, L. Neise, G. Peilert, A. Rosenhauer, H. Sorge, J. Aichelin, H. Stöcker, and W. Greiner, 1989, "Quantum molecular dynamics: a microscopic model from UNILAC to CERN energies," Nucl. Phys. A 495, 303.

Hartnack C., R.K. Puri, J. Aichelin, J. Konopka, S.A. Bass, H. Stöcker, and W. Greiner, 
1998, "Modelling the many-body dynamics of heavy ion collisions: Present status and future perspective," Eur. J. Phys. A 1, 151.

Heller E.J., 1975, "Time-dependent approach to semiclassical dynamics," J. Chem. Phys. 62,1544 .

Hemley R.J., and H.K. Mao, 1992, "Anomalous low-frequency excitations in diamond-cell studies of hydrogen at megabar pressures," Phys. Lett. A 163, 429.

W.G. Hoover, 1985, "Canonical dynamics: Equilibrium phase-space distributions," Phys. Rev. A 31, 1695.

Hoover W.G., 1986, "Molecular dynamics," Lecture Notes in Physics 258, (Springer, Berlin) Kanada-En'yo Y., H. Horiuchi, and A. Ono, 1995, "Structure of Li and Be isotopes studied with antisymmetrized molecular dynamics," Phys. Rev. C 52, 628.

Katz A., 1965, Classical Mechanics, Quantum Mechanics, Field Theory, (Academic Press, New York).

Kerman A.K., and S.E. Koonin, 1976, "Hamiltonian formulation of time-dependent variational principles for the many-body system," Ann. Phys. 100, 332.

Khoa D.T., N. Ohtsuka, M.A. Matin, A. Faessler, S.W. Huang, E. Lehmann, and R.K. Puri, 1992, "In-medium effects in the description of heavy-ion collisions with realistic NN interactions," Nucl. Phys. A 548, 102.

Kiderlen D., and P. Danielewicz, 1996, "Fragments in gaussian wave-packet dynamics with and without correlations," Nucl. Phys. A 620, 346.

Klakow D., C. Toepffer, and P.-G. Reinhard, 1994, "Hydrogen under extreme conditions," Phys. Lett. A 192, 55.

Klakow D., C. Toepffer, and P.-G. Reinhard, 1994, "Semiclassical molecular dynamics for strongly coupled Coulomb systems," J. Chem. Phys. 101, 1. 
Klauder J.R., and B.-S. Skagerstam, 1985, Coherent states, (World Scientific Publishing, Singapore).

Knaup, M., P.-G. Reinhard, and Ch. Toepffer, 1999, "Wave packet molecular dynamics simulations of hydrogen near the transition to a metallic fluid," Contrib. Plasma Phys. 39, 57.

Knoll J., and B. Strack, 1984, "The dynamics of the nuclear disassembly in a field-theoretical model at finite entropies," Phys. Lett. B 149, 45.

Knoll J., and J. Wu, 1988, "Expansion dynamics and multifragmentation of a saturating system of fermions," Nucl. Phys. A 481, 173.

Konopka J., H. Stöcker, and W. Greiner, 1995, "On the impossibility of temperature extraction from heavy ion induced particle spectra," Nucl. Phys. A 583, 357c.

Kramer P., and M. Saraceno, 1981, "Geometry of the time-dependent variational principle in quantum mechanics," Lecture Notes in Physics 140, (Springer, Berlin).

Kusnezov D., A. Bulgac, and W. Bauer, 1990, "Canonical ensembles from chaos," Ann. of Phys. 204, 155.

Kusnezov D., 1993, "Quantum ergodic wave functions from a thermal non-linear Schrödinger equation," Phys. Lett. A 184, 50.

Lacroix D., Ph. Chomaz, and S. Ayik, 1998, 1999, "Quantal extension of mean-field dynamics," Proceedings XXXVI. Int. Winter Meeting on Nuclear Physics, Bormio/Italy p. 485, and "On the simulation of extended TDHF theory," Nucl. Phys. A 651, 369.

Latora V., M. Belkacem, and A. Bonasera, 1994, "Dynamics of instabilities and intermittency," Phys. Rev. Lett. 73, 1765.

Lehmann E., R.K. Puri, A. Faessler, G. Batko, and S.W. Huang, 1995, "Consequences of a covariant description of heavy ion reactions at intermediate energies," Phys. Rev. C 51, 
2113.

Maruyama Toshiki, A. Ohnishi, and H. Horiuchi, 1992, "Evolution of reaction mechanisms in the light heavy-ion system," Phys. Rev. C 45, 2355.

Maruyama Toshiki, A. Ono, A. Ohnishi, and H. Horiuchi, 1992, "Fragment mass distribution in intermediate energy heavy ion collisions and the reaction time scale," Prog. Theor. Phys. $87,1367$.

Maruyama Toshiki, K. Niita, and A. Iwamoto, 1996, "Extension of quantum molecular dynamics and its application to heavy-ion collisions," Phys. Rev. C 53, 297.

Metropolis M., A.W. Metropolis, and M.N. Rosenbluth, A.H. Teller, and E. Teller, 1953, "Equation of state calculations by fast computing machines," J. Chem. Phys. 21, 1087.

Neff T., H. Feldmeier, R. Roth, and J. Schnack, 1999, "Realistic interactions and configuration mixing in fermionic molecular dynamics," Proc. Int. Workshop XXXVII in Hirschegg, ISSN 0720-8715, p. 283.

Niita K., S. Chiba, T. Maruyama, T. Maruyama, H. Takada, T. Fukahori, Y. Nakahara, and A. Iwamoto, 1995, "Analysis of the $(\mathrm{N}, \mathrm{xN}$ ') reactions by quantum molecular dynamics plus statistical model," Phys. Rev. C 52, 2620.

Neumann J.J. and G. Fai, 1994, "Classical lagrangian model of the Pauli principle," Phys. Lett. B 329, 419 .

Nosé S., 1984, "A unified formulation of the constant temperature molecular dynamics methods," J. Chem. Phys. 81, 511.

Nosé S., 1991, "Constant temperature molecular dynamics methods," Prog. of Theor. Phys. Suppl. 103, 1.

Ohnishi A., and J. Randrup, 1993, "Statistical properties of antisymmetrized molecular dynamics," Nucl. Phys. A 565, 474. 
Ohnishi A., and J. Randrup, 1995, "Incorporation of quantum statistical features in molecular dynamics," Phys. Rev. Lett. 75, 596.

Ohnishi A., and J. Randrup, 1997, "Inclusion of quantum fluctuations in wave packet dynamics," Ann. Phys. 253, 279.

Ohnishi A., and J. Randrup, 1997, "Quantum fluctuations affect the critical properties of noble gases," Phys. Rev. A 55, R3315.

Ono A., H. Horiuchi, Toshiki Maruyama, and A. Onishi, 1992, "Fragment formation studied with antisymmetrized version of molecular dynamics with two nucleon collisions," Phys. Rev. Lett. 68, 2898.

Ono A., H. Horiuchi, Toshiki Maruyama, and A. Onishi, 1992, "Antisymmetrized version of molecular dynamics with two-nucleon collisions and its application to heavy ion reactions," Prog. Theor. Phys. 87, 1185.

Ono A., H. Horiuchi, Toshiki Maruyama, and A. Onishi, 1993, "Fragment formation studied with antisymmetrized version of molecular dynamics with two-nucleon collisions," Phys. Rev. C 47, 2652.

Ono A., and H. Horiuchi, 1996, "Antisymmetrized molecular dynamics of wave packets with stochastic incorporation of Vlasov equation," Phys. Rev. C 53, 2958.

Ono A., and H. Horiuchi, 1996, "Statistical properties of AMD for non-nucleon-emission and nucleon-emission process," Phys. Rev. C 53, 2341.

Ono A., 1998, "Antisymmetrized molecular dynamics with quantum branching processes for collisions of heavy nuclei," Phys. Rev. C59, 853.

Peilert G., J. Randrup, H. Stöcker, and W. Greiner, 1991, "Clustering in nuclear matter at subsaturation densities," Phys. Lett. B 260, 271.

Peilert G., J. Konopka, M. Blann, M.G. Mustafa, H. Stöcker, and W. Greiner, 1992, ”Dy- 
namical treatment of Fermi motion in a microscopic description of heavy ion collisions," Phys. Rev. C 46, 1457.

Pochodzalla J., T. Möhlenkamp, T. Rubehn, A. Schüttauf, A. Wörner, E. Zude, M. Begemann-Blaich, T. Blaich, C. Gross, H. Emling, A. Ferrero, G. Imme, I. Iori, G.J. Kunde, W.D. Kunze, V. Lindenstruth, U. Lynen, A. Moroni, W.F.J. Müller, B. Ocker, G. Raciti, H. Sann, C. Schwarz, W. Seidel, V. Serfling, J. Stroth, A. Trzcinski, W. Trautmann, A. Tucholski, G. Verde, and B. Zwieglinski, 1995, "Probing the nuclear liquid-gas phase transition," Phys. Rev. Lett. 75, 1040.

Pochodzalla J., 1997, "The search for the liquid-gas phase transition in nuclei," Prog. Part. Nucl. Phys. 39, 443.

Pühlhofer F., 1977, "On the interpretation of evaporation residue mass distributions in heavy-ion induced fusion reactions," Nucl. Phys. A 280, 267.

Reinhard P.-G., and E. Suraud, "Stochastic TDHF and the Boltzmann-Langevin equation," Ann. Phys. (NY) 216, 98.

Saraceno M., P. Kramer, and F. Fernandez, 1983, "Time-dependent variational description of $\alpha \alpha$ scattering," Nucl. Phys. A 405, 88.

Schmidt H.-J., and J. Schnack, "Investigations on finite ideal quantum gases," Physica A 260, 479 .

Schnack J., and H. Feldmeier, 1996, "Statistical properties of Fermionic Molecular Dynamics," Nucl. Phys. A 601, 181.

Schnack J., and H. Feldmeier, 1997, "The nuclear liquid-gas phase transition within Fermionic Molecular Dynamics," Phys. Lett. B 409, 6.

Schnack J., 1996, "Kurzreichweitige Korrelationen in der Fermionischen Molekulardynamik," dissertation, Technical University Darmstadt. 
Schnack J., 1998, "Molecular dynamics investigations on a quantum system in a thermostat," Physica A 259, 49.

Schnack J., 1999, "Thermodynamics of the harmonic oscillator using coherent states," Europhys. Lett. 45, 647.

Sorge H., H. Stöcker, and W. Greiner, 1989, "Poincaré invariant hamiltonian dynamics: Modelling multi-hadronic interactions in a phase space approach," Ann. Phys. (NY) 192, 266.

Sorge H., 1995, "Flavor production in $\mathrm{Pb}(160 \mathrm{AGeV})$ on $\mathrm{Pb}$ collisions: Effect of color ropes and hadronic rescattering," Phys. Rev. C C52, 3291.

Stöcker H., and W. Greiner, 1986, "High-energy heavy ion collisions: probing the equation of state of highly excited hadronic matter," Phys. Rep. 137, 277.

Suarez Barnes I.M., M. Nauenberg, M. Nockleby, and S. Tomsovic, 1993, "Semiclassical theory of quantum propagation: The Coulomb potential," Phys. Rev. Lett. 71, 1961.

Topaler M.S., M.D. Hack, T.C. Allison, Yi-Ping Liu, S.L. Mielke, D.W. Schwenke, and D.G. Truhlar, 1997, "Validation of trajectory surface hopping methods against accurate quantum mechanical dynamics and semiclassical analysis of electronic-to-vibrational energy transfer," J. Chem. Phys. 106, 8699.

Tsue Y., and Y. Fujiwara, 1991, "Time-dependent variational approach in terms of squeezed coherent states," Prog. Theor. Phys. 86, 443.

Tully J.C., 1990, "Molecular dynamics with electronic transitions," J. Chem. Phys. 93, 1061.

Wilets L., E.M. Henley, M. Kraft, and A.D. MacKellar, 1977, "Classical many-body model for heavy-ion collisions incorporating the Pauli principle," Nucl. Phys. A 282, 341.

Wilets L., Y. Yariv, and R. Chestnut, 1978, "Classical many-body model for heavy-ion 
collisions (II)," Nucl. Phys. A 301, 359.

Wilets L., and J.S. Cohen, 1998, "Fermion molecular dynamics in atomic, molecular and optical physics," Contemporary Physics 39, 163.

Wolf Gy., G. Batko, W. Cassing, U. Mosel, K. Niita, and M. Schäfer, 1990, "Dilepton production in heavy-ion collisions," Nucl. Phys. A 517, 615. 